\title{
WIRE ROPE (EXPORT CLASSIFICATION)
}

\section{COMMERCIAL STANDARD CS154E-49}

Effective Date For New Production From February 1, 1949

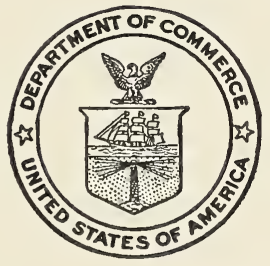

A RECORDED VOLUNTARY STANDARD OF THE TRADE

UNITED STATES DEPARTMENT OF COMMERCE CHARLES SAWYER, Secretary 


\section{COMMODITY STANDARDS}

Simplified Practice Recommendations and Commercial Standards are developed by manufacturers, distributors, and users in cooperation with the Commodity Standards Division of the National Bureau of Standards. The purpose of Simplified Practice Recommendations is to eliminate avoidable waste through the establishment of standards of practice for stock sizes and varieties of specific commodities that currently are in general production and demand. The purpose of Commercial Standards is to establish standard methods of test, rating, certification, and labeling of commodities, and to provide uniform bases for fair competition.

The adoption and use of a Simplified Practice Recommendation or Commercial Standard is voluntary. However, when reference to a Commercial Standard is made in contracts, labels, invoices, or advertising literature, the provisions of the standard are enforceable through usual legal channels as a part of the sales contract.

A Simplified Practice Recommendation or Commercial Standard originates with the proponent industry. The sponsors may be manufacturers, distributors, or users of the specific product. One of these three elements of industry submits to the Commodity Standards Division the necessary data to be used as the basis for developing a standard of practice. The Division, by means of assembled conferences or letter referenda, or both, assists the sponsor group in arriving at a tentative standard of practice and thereafter refers it to the other elements of the same industry for approval or for constructive criticism that will be helpful in making any necessary adjustments. The regular procedure of the Division assures continuous servicing of each effective Simplified Practice Recommendation and Commercial Standard, through review and revision, whenever, in the opinion of the industry, changing conditions warrant such action. Simplified Practice Recommendations and Commercial Standards are printed and made available by the Department of Commerce through the Government Printing Office.

\section{COMMERCIAL STANDARD FOR WIRE ROPE (EXPORT CLASSIFICATION)}

On August 4, 1948, at the instance of the Wire Rope Institute, a Recommended Commercial Standard for Wire Rope (Export Classification) was presented to manufacturers and exporters for written acceptance. Those concerned have since accepted and approved the standard as shown herein.

Project Manager: E. C. BARReTt, Commodity Standards Division, National Bureau of Standards.

Technical Adviser: A. H. Stang, Mechanics Division, National Bureau of Standards. 
Purpose

Scope

Definitions

General requirements

Detail requirements

Sizes

$6 \times 19$ classification wire rope (table 7 )

$6 \times 37$ classification wire rope (table 8$)$

$6 \times 7$ wire rope (table 9)

$8 \times 19$ wire rope (table 10$)$

Flattened strand ropes, $6 \times 25 ; 6 \times 30$ (table 11$) \ldots$

Flattened strand rope, $6 \times 8$ (table 12$) \ldots \ldots$

Galvanized highway guard cable (table 13)

$6 \times 24$ Galvanized steel mooring line (table 14)

Flat wire rope (table 15)

$18 \times 7$ nonrotating wire rope $($ table 16$)$

$6 \times 42$ tiller rope (table 17)

Locked coil track strand (table 18)

Smooth coil track strand (table 19)

$6 \times 12$ galvanized running rope (table 20 )

Marline clad rope, $5 \times 19.5 \times 25$ (table 21$)$

Coke still rope, $7 \times 19(6 \times 19$ with wire strand core $)($ table 22$) \ldots . .29$

Torpedo lines, $5 \times 5$ (table 23)

Galvanized steel strand, 7-wire strand (table 24)

Galvanized steel strand, 19-wire strand (table 25) 31

Galvanized steel strand, utilities grade, 3- and 7-wire strand (table 26)

Galvanized bridge strand (table 27) $\ldots \ldots \ldots \ldots \ldots$

Galvanized bridge rope (table 28)

$6 \times 7$ galvanized iron guy rope (table 29$) \ldots$

Galvanized spring lay rope (table 30 ) $\ldots \ldots \ldots$

$6 \times 37$ galvanized steel hawser (table 31 )

$6 \times 19$ marline clad grain shovel rope (table 32 )

$9 \times 4$ galvanized mast arm rope (table 33 ) . . . . . . . . . .

$6 \times 7$ sash cord (table 34)

$6 \times 7$ aircraft cord (table 35$)$

$7 \times 7$ aircraft cord (table 36)

$6 \times 19$ aircraft cord with $7 \times 7$ IWRC (table 38$)$

$7 \times 19$ aircraft cord (table 39)

19-wire aircraft strand (table 40) 37

$8 \times 19$ elevator rope (table 41) 38

$6 \times 19$ elevator rope (table 42) 38

General recommendations _...

Marking and certification 45

Glossary of technical terms

Effective date

History of project

Standing committee

Appendix

Acceptance of Commercial Standard

To the acceptor

Acceptors

List of Commercial Standards 57 

for

\section{WIRE ROPE (EXPORT CLASSIFICATION)}

\section{PURPOSE}

1. The purpose of this standard is to provide a nationally recognized minimum specification for wire rope as a basis for better understanding between manufacturers and exporters and the buyers in the foreign market to assure the purchaser of a quality product as exported from the United States of America.

\section{SCOPE}

2. This standard provides minimum requirements of material, construction, and test methods for wire rope as manufactured in the United States. It also provides definitions, together with a glossary of terms, useful in the trade for better understanding between buyer and seller. Tables 7 to 42 show those grades and sizes of wire rope that are considered standard with the industry to meet the greatest needs.

3. The numbers, sizes, and grades of wire and the ways in which the wires can be combined into rope result in a possible wide number of types and range of sizes. Manufacturers have simplified their lines and standardized on those types and sizes most in demand. The wire ropes covered in this commercial standard represent those sizes and types usually carried in stock by manufacturers for immediate shipment. Selection is therefore simplified and uniform quality is assured. Other grades, sizes, and constructions may be available by arrangement with the supplier.

\section{DEFINITIONS ${ }^{1}$}

4. Strand. - An assembly of wires laid helically around a center in one or more symmetrical layers.

4a. Center. - The material in the axis of a strand about which the wires are laid.

4b. Helix, right-hand.-The outer wires form a helix about the center similar to the threads on a right-hand screw.

4c. Helix, left-hand.-The outer wires form a helix about the' center similar to the threads on a left-hand screw.

4d. Operation. - All the wires laid in the same operation have the same pitch and, therefore, each wire is in contact with the same adjacent wires for its entire length. All the wires in each layer are laid in the same operation. If two layers are laid in the same operation, each wire in the upper layer is in contact not only with adjacent

\footnotetext{
${ }^{1}$ See also glossary, page 45 .
} 
wires in the same layer, but also with adjacent wires in the layer beneath. If a wire in one layer is not in contact for its entire length with the same adjacent wires in the layer beneath, the layers were laid in different operations.

4e. Pitch.- The distance, parallel to the axis of the strand, in which a wire makes one complete turn about the center.

5. Wire.

5a. Filler.-The wires having for their primary purpose the spacing and support of the main wires.

5b. Galvanized.-Wire that is coated with zinc.

5c. Layer.-A group of adjacent wires approximately the same distance from the perimeter of the strand. The center is not a layer.

5d. Main.-The wires carrying all or the greater part of the load on the wire rope.

5e. One diameter.- The wires in a layer are of one diameter provided the difference between the diameter of the smallest wire and the diameter of the largest wire does not exceed the value given in table 1.

TABLE 1. Wires of one diameter

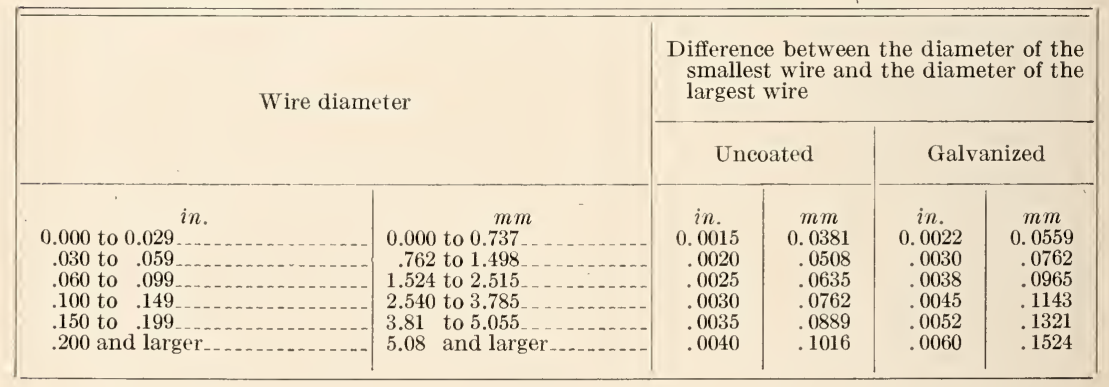

5f. Uncoated.-Wire that is not galvanized (zinc coated). An uncoated wire may be coated with other material, if desired by the contractor, provided the diameter is not increased more than 0.001 inch and provided the coating is not detrimental to the appearance or serviceableness of the wire rope.

6. Wire rope.-A group of strands laid helically around a core.

6a. Core.-The material in the axis of a wire rope about which the strands are laid.

6b. Construction.-Number and arrangement, of wires in strands and number of strands. Also, if rope has metal core, the number and arrangement of wires in core.

6c. Diameter, actual.-The measured diameter of the circumscribed circle enclosing the strands.

6d. Diameter, nominal.-The diameter stated in the order.

6e. Helix, right-hand.-The strands form a helix about the core similar to the threads on a right-hand screw.

6f. Helix, left-hand.- The strands form a helix about the core similar to the threads on a left-hand screw.

6g. Lay, lang. - The direction of the helix of the strands about the rope core is the same as the direction of the helix of the wires about the strand center. 
6h. Lay, regular.-The direction of the helix of the strands about the rope core is opposite to the direction of the helix of the wires about the strand center.

6i. Pitch.-The distance, parallel to the axis of the rope, in which a strand makes one complete turn about the core.

6j. Preformed.-The strands are permanently shaped, before fabrication into the rope, to the helical form they assume in the wire rope.

$6 \mathrm{k}$. Not preformed. - Neither the strands nor the wire rope has been subjected to a mechanical operation, the primary purpose of which is to preform either the strands or the wire rope.

\section{GENERAL REQUIREMENTS}

7. Wire rope shall be made from the best quality of the specified grade of material, shall be of good workmanship, and shall be free from defects that might be detrimental to its appearance or serviceableness.

8. The main wires of a wire rope shall be one of the following materials designated commercially as improved plow steel, plow steel, mild plow steel, traction steel, highway-guard steel, annealed steel, iron, or corrosion-resistant steel.

9. Galvanized wire shall be uniformly and continuously coated with zinc, which adheres firmly to the wire. The zinc shall be applied to each wire either by the electrodeposition process or by what is designated commercially as "hot process galvanizing."

10. Grades of wire rope.-The wide and varied uses of wire rope, together with the conditions of its use, require a rope that will meet demands for strength, toughness, ability to withstand abrasion and crushing, and to resist bending fatigue in varying degrees. The following grades of wire rope are offered to meet appropriate conditions:

10a. Improved plow steel is the strongest, toughest, and most wearresistant of all standard grades of wire rope.

10b. Plow steel is an intermediate grade of wire rope used where service requirements do not demand the improved plow steel grade.

10c. Mild plow steel wire rope is used in installations where strength and resistance to abrasion are not so important.

$10 \mathrm{~d}$. Traction steel is a special grade of wire rope designed for use on electric traction type elevators.

10e. Iron is a grade of wire rope with limited use due to a low tensile strength and low abrasion resistance.

10f. Corrosion-resistant steel, which is an alloy steel containing approximately 18 percent of chromium and 8 percent of nickel, is used where tensile strength and corrosion resistance are important.

11. Wire rope cores.-Fiber cores are standard for most of the constructions of wire rope. The fiber core forms the heart of the rope, supports the strands, provides internal lubrication, and contributes to the flexibility of the rope.

12. Wire cores are made of either independent wire rope or wire strand. They increase resistance to crushing and make a stronger 
rope. They are recommended where heat is present sufficient to destroy fiber cores. They are less resilient than fiber core ropes; this smaller amount of stretch makes them suitable for bridges and applications where the amount of elongation is important. They are less suitable for operating duty where shock loads are frequent. Figure 1 shows the types of core construction.

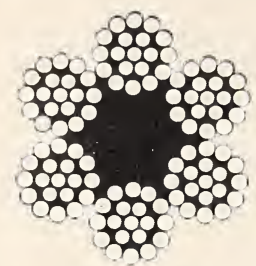

Fiber core

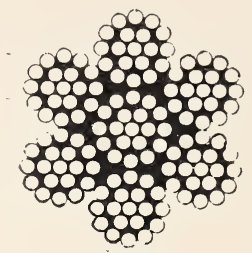

Strand core

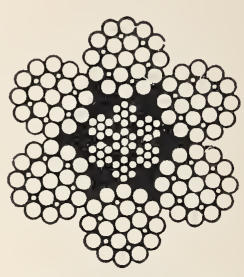

Wire rope core

Figure 1. Wire rope cores.

13. Wire rope lays. - The lay of a wire rope is determined by the direction of the wires in each strand and the direction of the strand in the rope.

WIRE ROPE LAYS

Right regular lay: The wires in the strand are laid to the left and each strand is laid to the right. (See fig. 2, A.)

Left regular lay: The wires in the strand are laid to the right and each strand is laid to the left. (See fig. 2, B.)

Right lang lay: Both the wires in the strand and the strands in the rope are laid to the right. (See fig. 2, C.)

Left lang lay: Both the wires in the strand and the strands in the rope are laid to the left. (See fig. 2, D.)

Alternate (or reverse) lay: Alternate left and right lay strands. (See fig. 2, E.) 

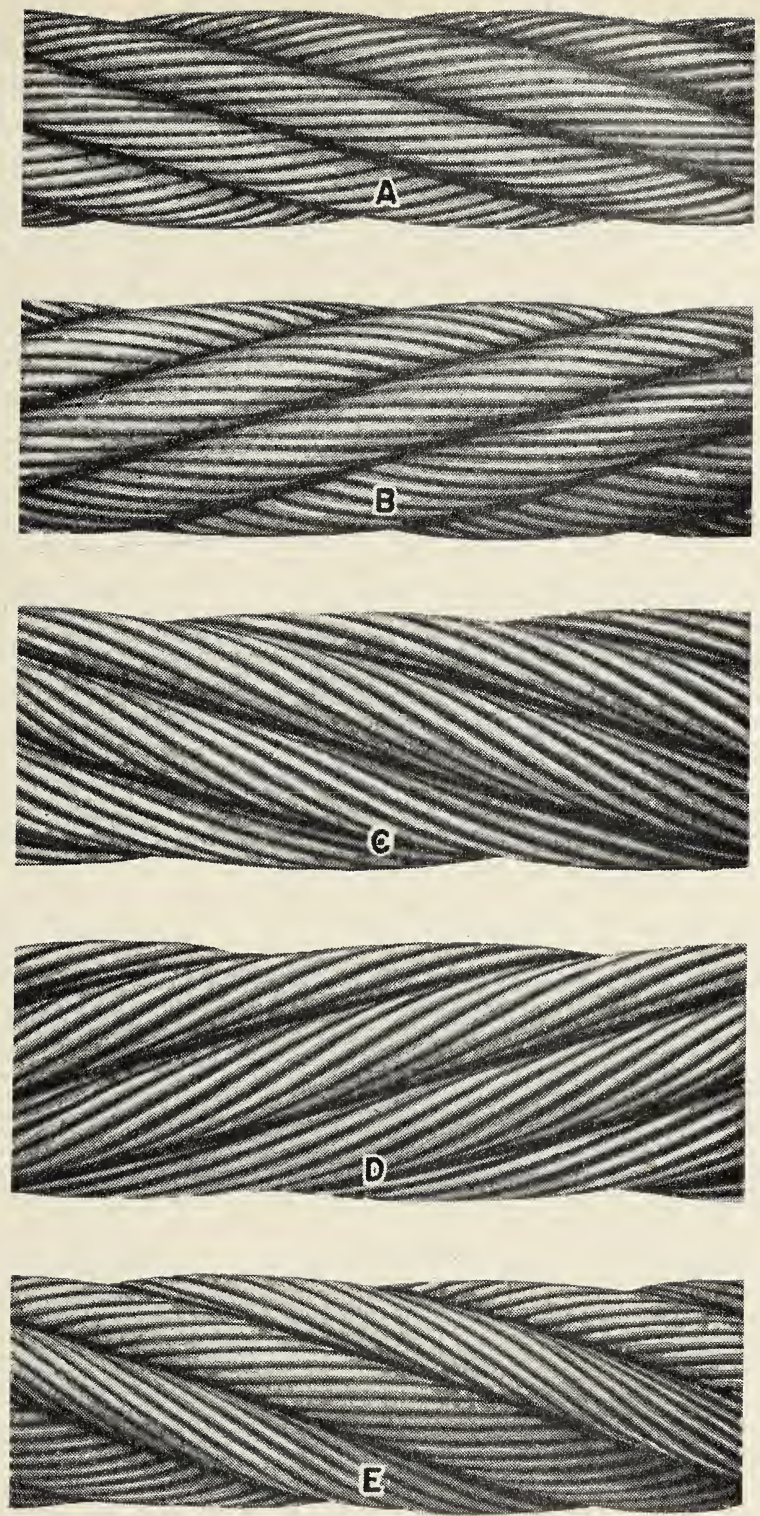

Figure 2. Wire rope lavs 
14. Wire-rope constructions.-To show the wide variations in wirerope constructions that are considered standard with the industry, the following illustrations are given:
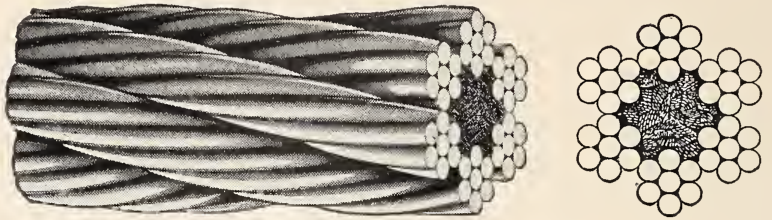

$6 \times 7$ Fiber core.
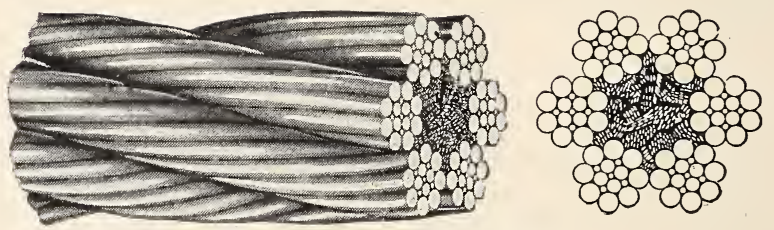

$6 \times 17$ Filler wire fiber core.
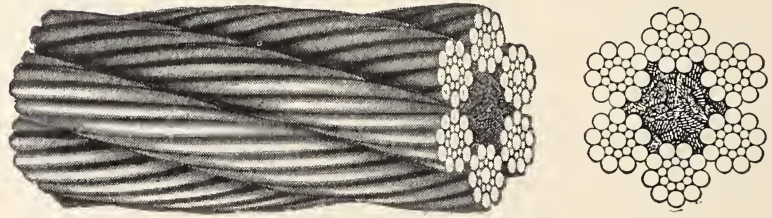

$6 \times 19$ Seale fiber core.
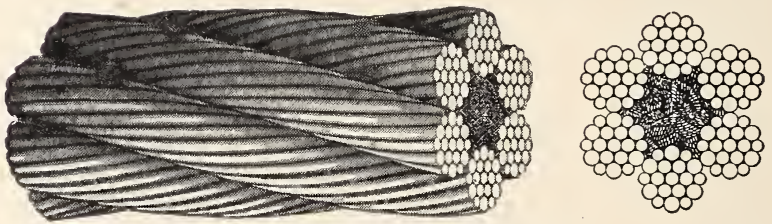

$6 \times 19$ Warrington fiber core.
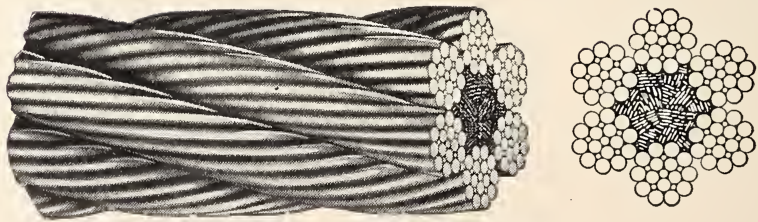

$6 \times 21$ Filler wire fiber core. 

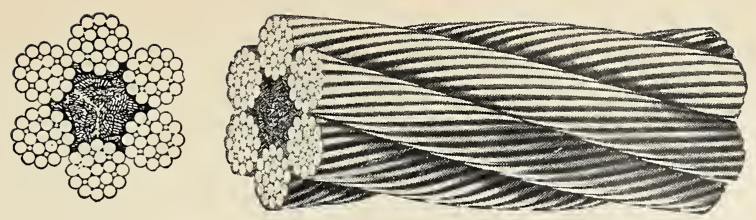

$6 \times 25$ Filler wire fiber core.
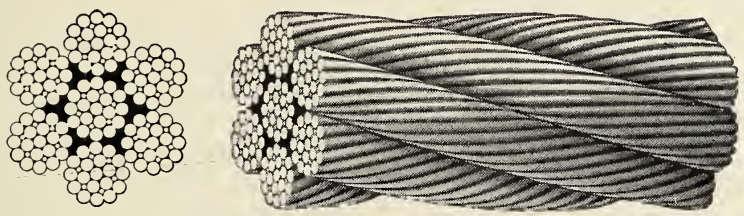

$6 \times 25$ Filler wire strand core.
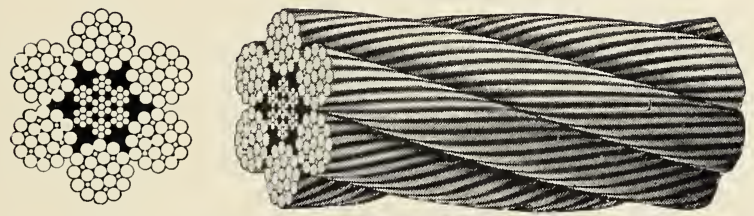

$6 \times 25$ Filler wire independent wire rope core.
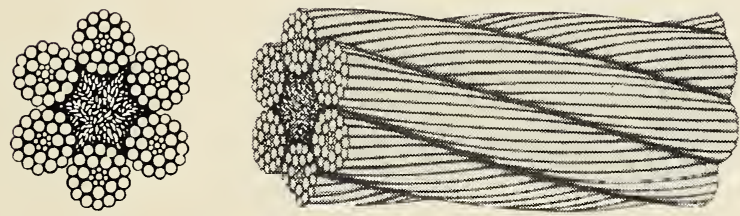

$6 \times 29$ Warrington fiber core.
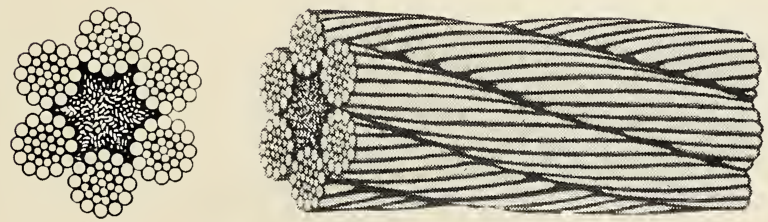

$6 \times 31$ Seale fiber core. 

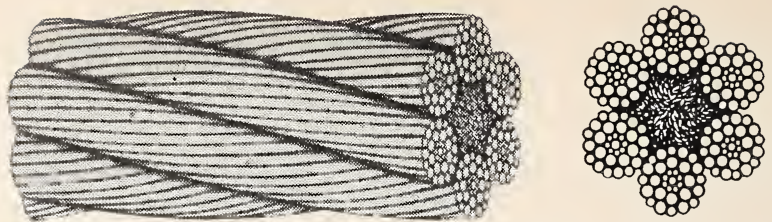

$6 \times 33$ Warrington fiber core.
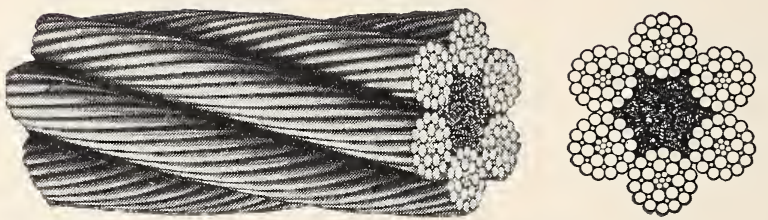

$6 \times 35$ Filler wire fiber core.
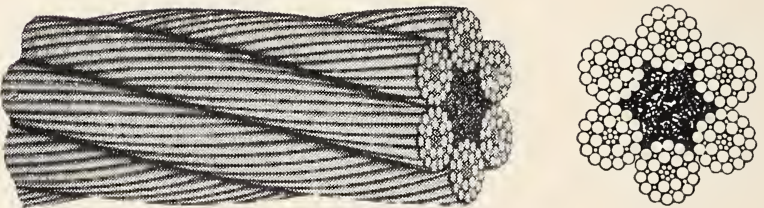

$6 \times 36$ Filler wire fiber core.
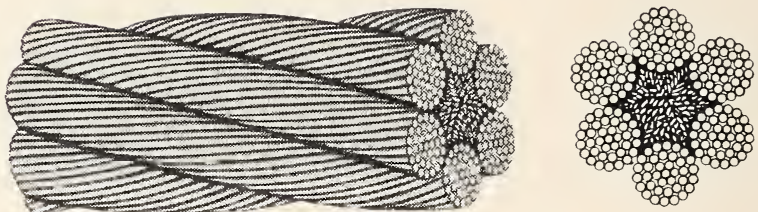

$6 \times 37$ (3 operations) fiber core.
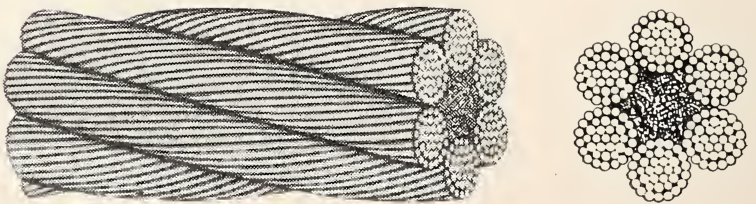

$6 \times 37$ ( 2 operations) fiber core. 

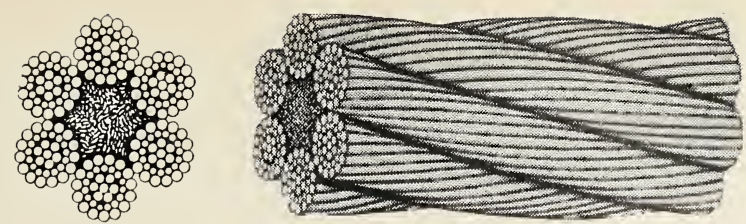

$6 \times 37$ Seale fiber core.
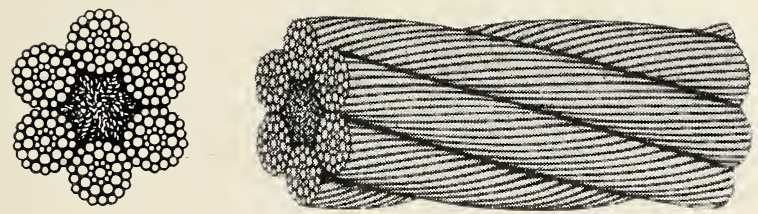

$6 \times 37$ Warrington fiber core.
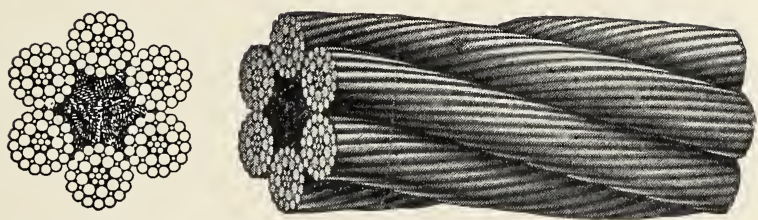

$6 \times 39$ Filler wire fiber core.
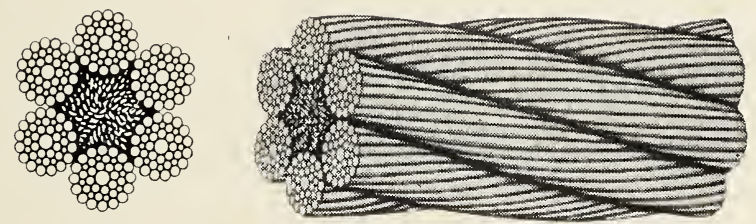

$6 \times 41$ Warrington Seale fiber core.
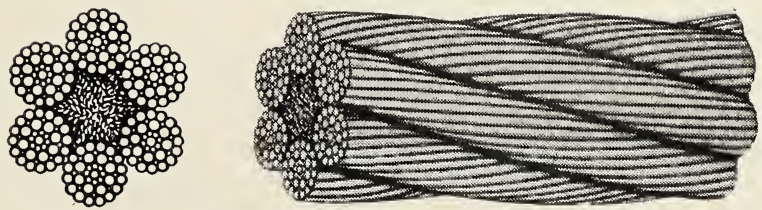

$6 \times 41$ Filler wire fiber core. 

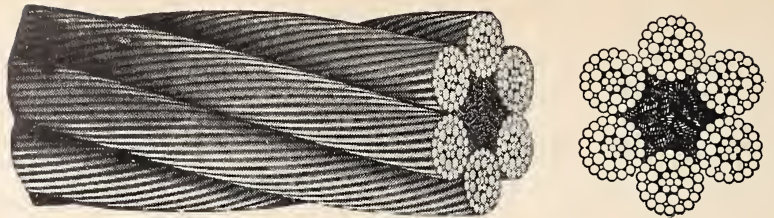

$6 \times 43$ Filler wire fiber core.
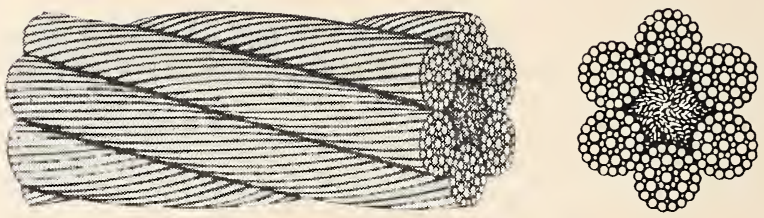

$6 \times 46$ Filler wire fiber core.
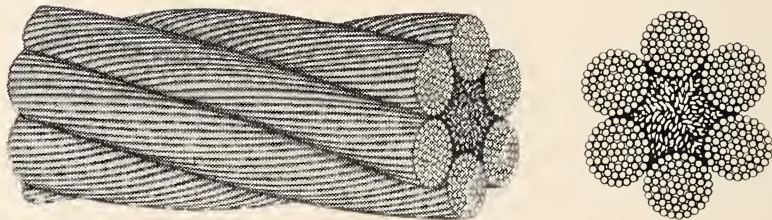

$6 \times 61$ (4 operations) fiber core.
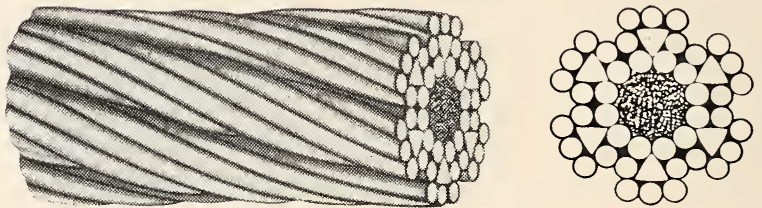

$6 \times 8$ Flattened strand-Type D fiber core.
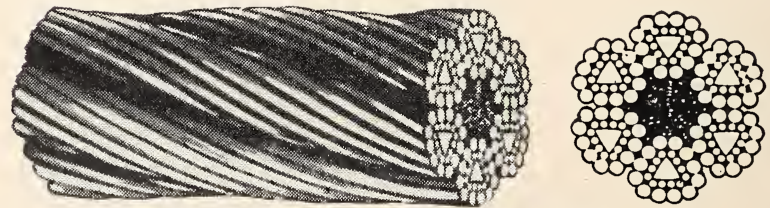

$6 \times 25$ Flattened strand-Type B fiber core. 

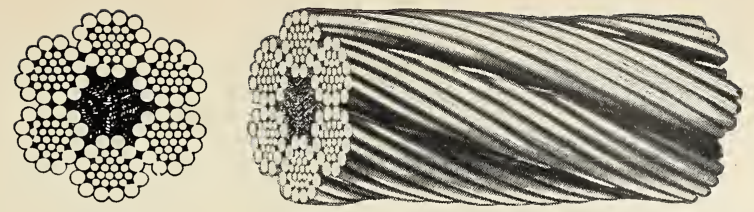

$6 \times 30$ Flattened strand-Type G fiber core.
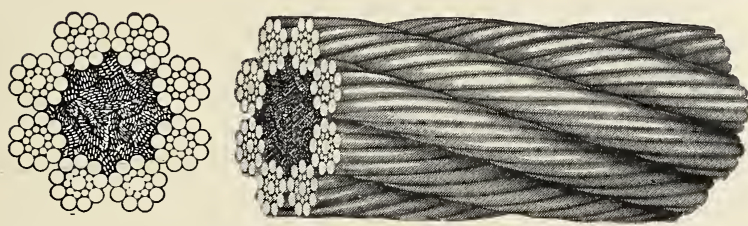

$8 \times 19$ Seale fiber core.
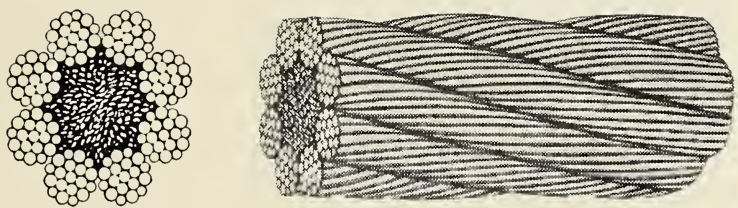

$8 \times 19$ Warrington fiber core.
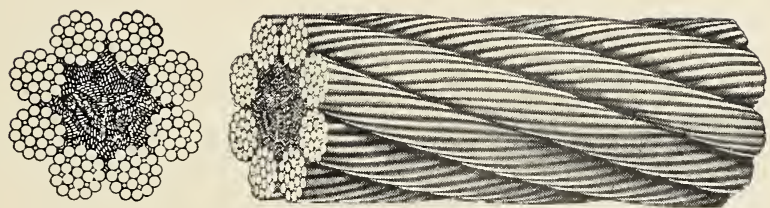

$8 \times 25$ Filler wire fiber core.
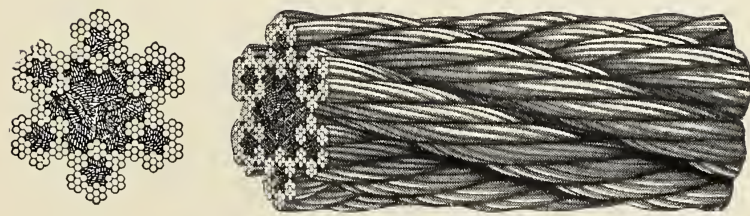

$6 \times 42$ Tiller rope. 


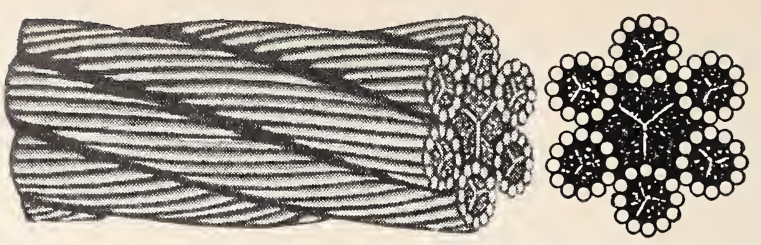

$6 \times 12$ Galvanized running rope fiber core.
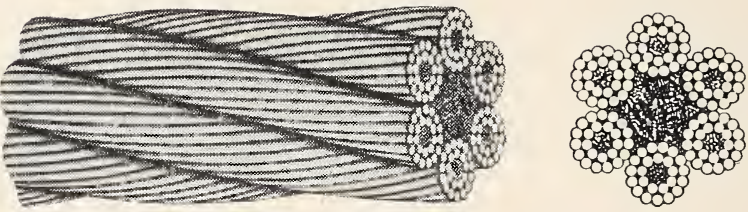

$6 \times 24$ Fiber core -6 fiber centers.
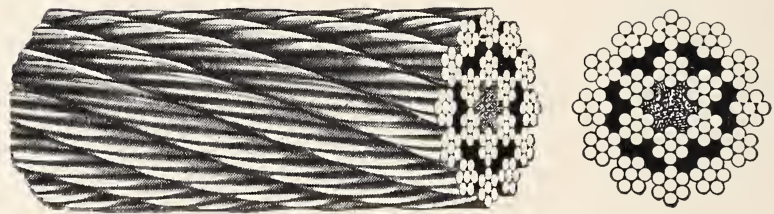

$18 \times 7$ Nonrotating fiber core.
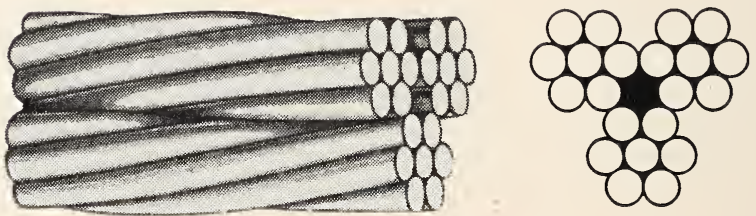

$3 \times 7$ Highway guard cable.
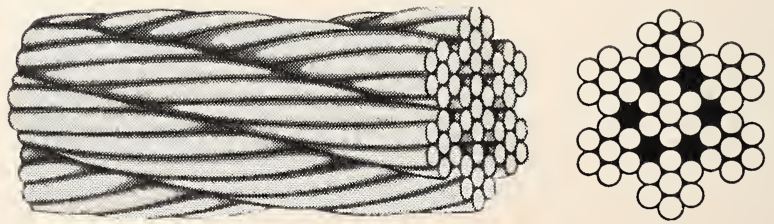

$7 \times 7$ Highway guard cable. 

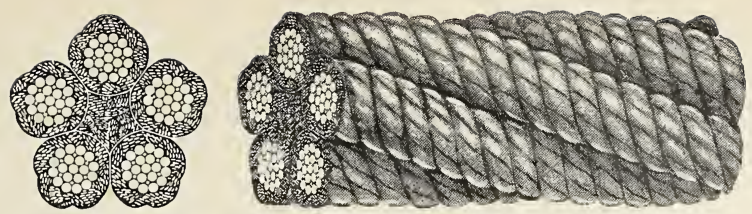

$5 \times 25$ Filler wire-Marline clad fiber core.
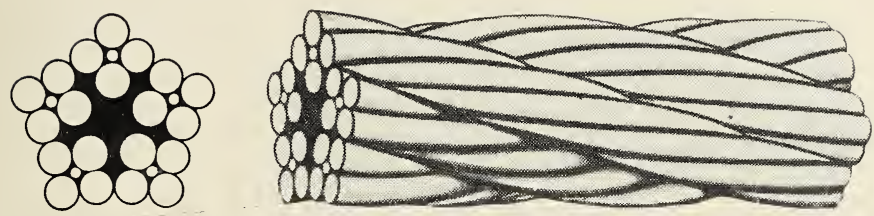

$5 \times 5$ Torpedo line fiber core.

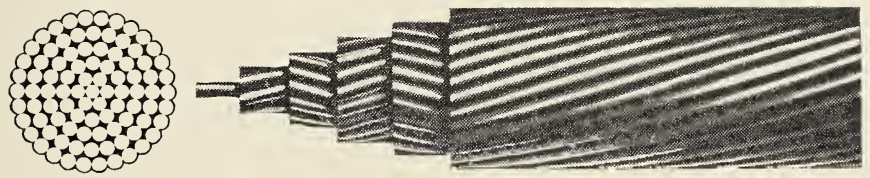

Lock coil track strand.
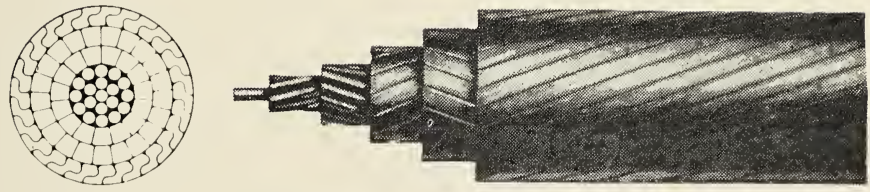

Smooth coil track strand.

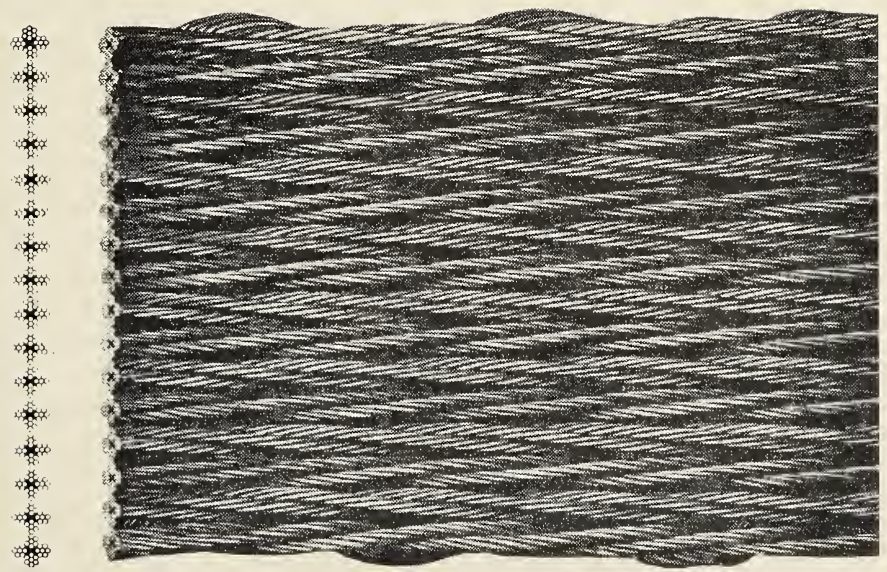

Flat wire rope. 
15. Physical properties.

15a. Abrasion.--In certain applications of wire rope, abrasion is the greatest destructive force; therefore, in the selection of a wire rope the conditions of use should be considered to give maximum service. Some ropes are designed especially to resist abrasion. (See fig. 3.)

15b. Bending fatigue.-When a wire rope is to be subjected to sharp or repeated bending, an especially designed rope to minimize fatigue should be used. It would be desirable, under these conditions, to specify the use of a preformed wire rope.

Figure 3 shows the resistance to bending fatigue and resistance to abrasion of a few constructions of wire rope.

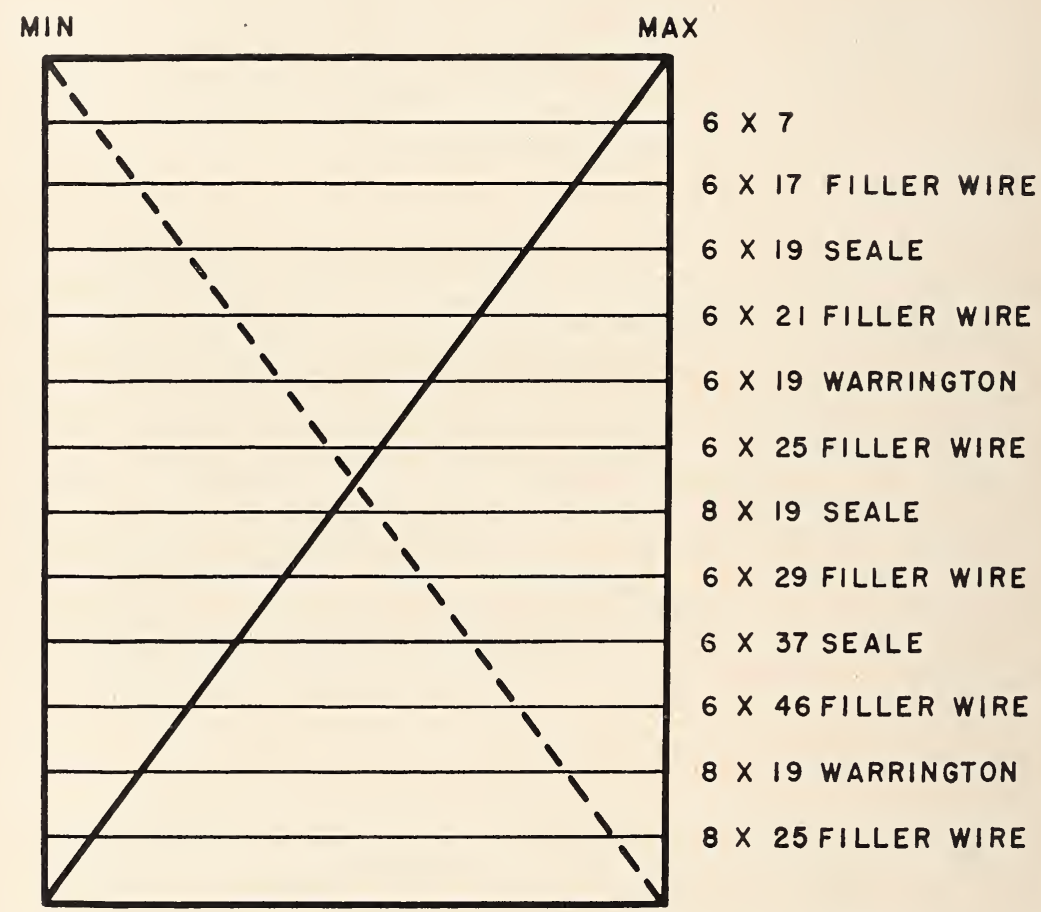

Figure 3. Resistance to abrasion and bending fatigue.

-, Resistance to abrasion; --.-----, resistance to bending fatigue.

15c. Crushing.-Often wire rope is subjected to conditions tending to crush or distort the rope. This should be minimized by the selection of the proper rope to meet the exacting conditions. An independent wire rope core or a wire strand core can be used in these cases with proper consideration to its effect on other properties which may be equally desirable.

15d. Tensile strength.-The tensile strength of the wire used in the manufacture of wire rope is determined from the strength of the main 
wires of the rope. Main wires, except triangular wire centers, taken from the finished rope or seizing strand shall be in accordance with table 2 .

TABLE 2. Tensile strength of main wires

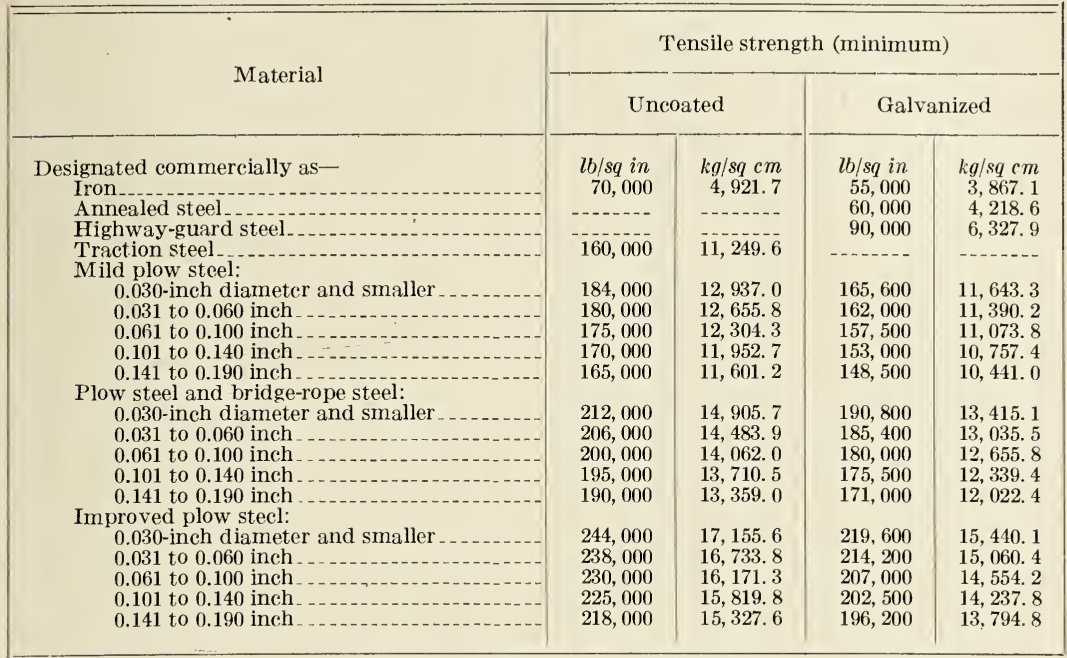

\section{5e. Breaking strength.}

(1) Actual breaking strength of wire rope supplied by United States manufacturers shall be that strength required to break the rope under tension.

(2) Aggregate breaking strength of wire rope, sometimes used by others, is arrived at by considering the breaking strength of the rope as the aggregate breaking strength of its component wires. ${ }^{2}$

(3) Table 3 shows the manner of converting from aggregate strength of rope to actual strength, and from actual to aggregate strength.

TABLE 3. Conversion of breaking strength methods for rope and strand constructions

\begin{tabular}{|c|c|c|}
\hline Rope constructions & $\begin{array}{c}\text { From aggregatc } \\
\text { strength to actual } \\
\text { strength, nuultiply } \\
\text { by- }\end{array}$ & $\begin{array}{l}\text { From actual } \\
\text { strength to aggre- } \\
\text { gate strength, } \\
\text { multiply by- }\end{array}$ \\
\hline $\begin{array}{l}6 \times 7 \\
6 \times 197,8 \times 19,6 \times 37 \text { hawser } \\
6 \times 42,18 \times 7 \\
6 \times 8 \text { flattened strand } \\
6 \times 25 \text { and } 6 \times 30 \text { flattened strand } \\
5 \times 19 \text { marline clad, } 6 \times 12 \text { running } \\
6 \times 24 \text { haw } \\
3-\text { and } 7 \text {-wire } \text { strand } \text { rope } \\
19 \text {-wire strand }\end{array}$ & $\begin{array}{r}0.860 \\
.825 \\
.800 \\
.720 \\
.885 \\
.850 \\
.900 \\
.875 \\
.960 \\
.940\end{array}$ & $\begin{array}{l}\text { 1. } 16279 \\
\text { 1. } 21212 \\
\text { 1. } 2500 \\
\text { 1. } 38889 \\
\text { 1. } 12994 \\
\text { 1. } 17647 \\
\text { 1. } 11111 \\
\text { 1. } 14286 \\
\text { 1. } 04167\end{array}$ \\
\hline
\end{tabular}

2 This method is not used by United States manufacturers. It is an easy way to determine the strength. but its disadvantage is that the resulting figure is only theoretical and is always higher than the actual breaking strength. 
15f. Tensile strength of wire rope shall be determined as outlined in paragraph 17.

15g. Rope diameters.-Diameters of all types and grades of wire rope shall be the nominal diamcter of the circumscribing circle expressed in inches or millimeters. Tolerances for wire rope diameters shall be as shown in table 4 .

TABLE 4. Diameter tolerances for wire rope

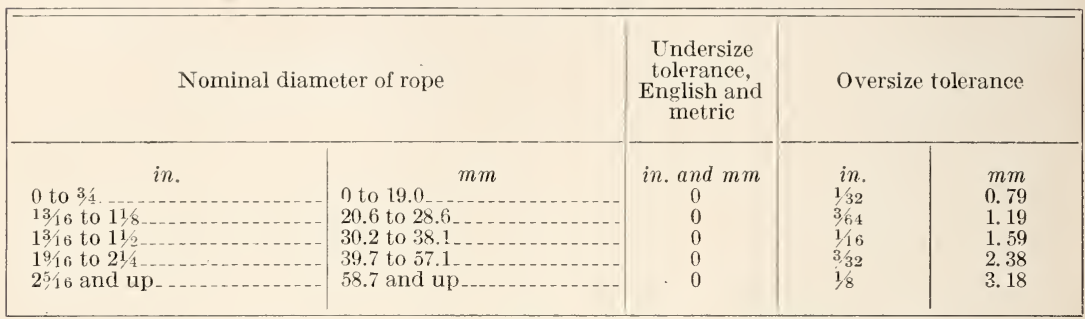

15h. Weights.-Weight of wire rope shall be expressed in pounds per linear foot or kilograms per meter.

15i. Metallic area.-The metallic area of a wire rope or strand is the sum of the cross sectional areas of the individual wires of which it is made. A method of calculating the approximate metallic area for a few rope constructions is given in table 5, where "d" is the rope diameter in inches or millimeter's. If the rope diameter " $d$ " is expressed in inches or millimeters, the area will be expressed in square inches or square millimeter's, respectively.

TABLE 5. Metallic area of wire rope

\begin{tabular}{|c|c|}
\hline Rope construction & Area \\
\hline $\begin{array}{l}6 \times 7 \\
6 \times 17 \\
6 \times 21 \text { filler wire } \\
6 \times 19 \text { Warrington. } \\
6 \times 25 \text { filler wire } \\
6 \times 37 \\
6 \times 25 \text { flattened strand, styles } \mathrm{B} \text { and } \mathrm{G} \\
8 \times 19\end{array}$ & $\begin{array}{c}\text { sq in. } \text { or } s q m m \\
0.380 \mathrm{~d}^{2} \\
.385 \mathrm{~d}^{2} \\
.395 \mathrm{~d}^{2} \\
.395 \mathrm{~d}^{2} \\
.405 \mathrm{~d}^{2} \\
.405 \mathrm{~d}^{2} \\
.400 \mathrm{~d}^{2} \\
.440 \mathrm{~d}^{2} \\
.352 \mathrm{~d}^{2}\end{array}$ \\
\hline
\end{tabular}

16. Safety factor.-The factor of safety is the ratio of the actual breaking strength of the wire rope to the working load. A wire rope with an actual breaking strength of $20,000 \mathrm{lb}(9072 \mathrm{~kg})$ and a total working load of $4,000 \mathrm{lb}(1814.4 \mathrm{~kg})$ would be operating with a safety factor of five.

16a. The proper factor of safety to use will be affected by many conditions such as speed of operation, type of fittings, acceleration and deceleration, size and location of sheaves and drums, and many others. Table 6 and table 6 a showing minimum safety factors are intended only as a guide. 
TABLE 6. Safety factors for typical services

\begin{tabular}{|c|c|}
\hline Type of service & Minimum safety factor \\
\hline $\begin{array}{l}\text { Track cables. } \\
\text { Mine shafts. } \\
\text { Miscellaneous hoisting equipment } \\
\text { Haulage ropes } \\
\text { Jib and pillar cranes cranes } \\
\text { Derricks. } \\
\text { Small electric and air hoists. } \\
\text { Hot ladle cranes } \\
\text { Elevators. }\end{array}$ & $\begin{array}{l}3.2 \text {. } \\
3.5 \text {. } \\
8.0 \text { for depths to } 500 \mathrm{ft}(152.40 \mathrm{~m}) \text {. } \\
7.0 \text { for depths } 500 \text { to } 1,000 \mathrm{ft}(152.40 \text { to } 304.80 \mathrm{~m}) \text {. } \\
6.0 \text { for depths } 1,000 \text { to } 2,000 \mathrm{ft}(304.8 \text { to } 609.6 \mathrm{~m}) \text {. } \\
5.0 \text { for depths } 2,000 \text { to } 3,000 \mathrm{ft}(609.6 \text { to } 914.4 \mathrm{~m}) \text {. } \\
4.0 \text { for depths } 3,000 \mathrm{ft} \text { and more }(914.4 \mathrm{~m} \text { and more). } \\
5.0 \text {. } \\
6.0 \text {. } \\
6.0 \text {. } \\
6.0 \text {. } \\
6.0 \text {. } \\
7.0 \text {. } \\
8.0 \text {. } \\
8.0 \text {. } \\
\text { See table } 6 \text { for safety factors for elevators. }\end{array}$ \\
\hline
\end{tabular}

TABLE 6a. Safety factors for elevators (based on static loading)

\begin{tabular}{|c|c|c|c|c|}
\hline \multicolumn{2}{|c|}{ Elevator car speed } & \multicolumn{3}{|c|}{ Minimum safety factors } \\
\hline $\begin{array}{l}\text { Feet per } \\
\text { minute }\end{array}$ & $\begin{array}{l}\text { Meters per } \\
\text { minute }\end{array}$ & Passenger & Freight & $\begin{array}{l}\text { Dumb-wait- } \\
\text { ers }\end{array}$ \\
\hline $\begin{array}{r}50 \\
100 \\
150 \\
200 \\
250\end{array}$ & $\begin{array}{l}15.24 \\
30.48 \\
45.72 \\
60.96 \\
76.20\end{array}$ & $\begin{array}{l}7.50 \\
7.85 \\
8.20 \\
8.54 \\
8.86\end{array}$ & $\begin{array}{l}6.67 \\
7.00 \\
7.32 \\
7.64 \\
7.92\end{array}$ & $\begin{array}{l}5.33 \\
5.66 \\
5.98 \\
6.29 \\
6.59\end{array}$ \\
\hline $\begin{array}{l}300 \\
350 \\
400 \\
450 \\
500\end{array}$ & $\begin{array}{r}91.44 \\
106.68 \\
121.92 \\
137.16 \\
152.40\end{array}$ & $\begin{array}{r}9.17 \\
9.47 \\
9.75 \\
10.01 \\
10.25\end{array}$ & $\begin{array}{l}8.20 \\
8.45 \\
8.70 \\
8.93 \\
9.14\end{array}$ & $\begin{array}{l}6.88 \\
7.18 \\
7.46 \\
7.74 \\
8.00\end{array}$ \\
\hline $\begin{array}{l}550 \\
600 \\
700 \\
800 \\
900\end{array}$ & $\begin{array}{l}167.64 \\
182.88 \\
213.36 \\
243.89 \\
274.30\end{array}$ & $\begin{array}{l}10.47 \\
10.68 \\
11.00 \\
11.25 \\
11.44\end{array}$ & $\begin{array}{r}9.32 \\
9.50 \\
9.78 \\
10.02 \\
10.21\end{array}$ & 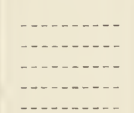 \\
\hline $\begin{array}{l}1,000 \\
1,100 \\
1,200 \\
1,300 \\
1,400\end{array}$ & $\begin{array}{l}304.80 \\
335.28 \\
365.76 \\
396.24 \\
426.73\end{array}$ & $\begin{array}{l}11.57 \\
11.67 \\
11.75 \\
11.81 \\
11.85\end{array}$ & $\begin{array}{l}10.34 \\
10.43 \\
10.50 \\
10.54 \\
10.58\end{array}$ & 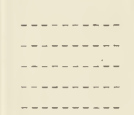 \\
\hline 1,500 & 457.20 & 11.87 & 10.61 & . \\
\hline
\end{tabular}

17. Methods of Test.

17a. Wires.-When testing the individual wires for tensile strength, tests shall be conducted in accordance with the current Standard Methods of Tension Testing of Metallic Materials, American Society for Testing Materials Designation E8.

$17 \mathrm{~b}$. Wire rope.-When testing the finished wire rope for its breaking strength, tensile test specimens shall be cut and suitable sockets or end fittings shall be attached.

$17 \mathrm{c}$. The length of the test specimen shall not be less than 3 feet between sockets for wire rope up to 1 inch $(25.4 \mathrm{~mm})$ in diameter, inclusive, and not less than 5 feet $(1.52 \mathrm{~m})$ between sockets for wire ropes $1 \frac{1}{8}$ inch $(28.6 \mathrm{~mm})$ in diameter and larger.

\section{DETAIL REQUIREMENTS}

\section{SIZES}

18. Wire-rope sizes.-The sizes of wire rope considered standard with the industry are shown in the following tables. The tables show the construction, diameter, weights, and strength of each rope. 


\begin{tabular}{|c|c|c|c|c|c|c|}
\hline \multirow{6}{*}{ 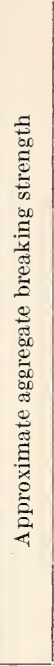 } & \multirow{2}{*}{ 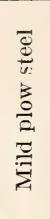 } & 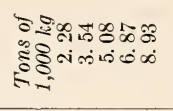 & 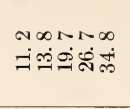 & 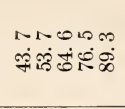 & 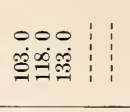 & \\
\hline & & 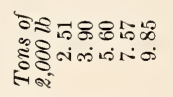 & 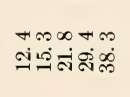 & 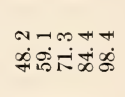 & 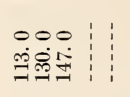 & \\
\hline & \multirow{2}{*}{ 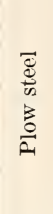 } & 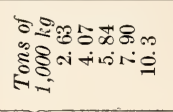 & $\begin{array}{l}00 \infty \infty 0 \\
\text { ற் }\end{array}$ & 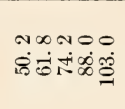 & 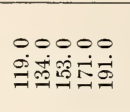 & 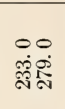 \\
\hline & & 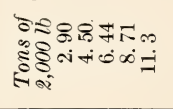 & 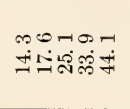 & 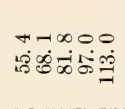 & 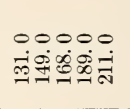 & 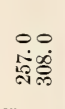 \\
\hline & \multirow{2}{*}{ 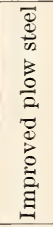 } & 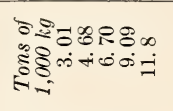 & 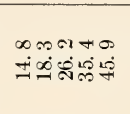 & 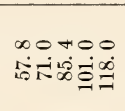 & 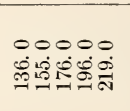 & 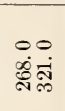 \\
\hline & & 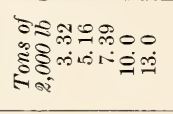 & 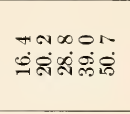 & 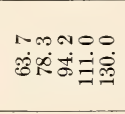 & 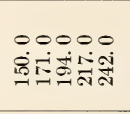 & 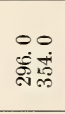 \\
\hline \multirow{6}{*}{ 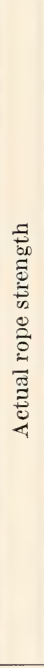 } & \multirow{2}{*}{ 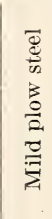 } & 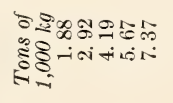 & 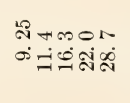 & 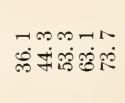 & 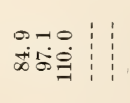 & \\
\hline & & 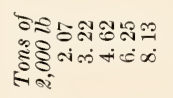 & 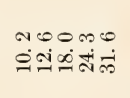 & 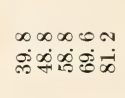 & 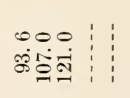 & \\
\hline & \multirow{2}{*}{$\begin{array}{l}\vec{\Phi} \\
\frac{\Phi}{0} \\
\text { है } \\
\vec{a}\end{array}$} & 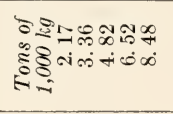 & 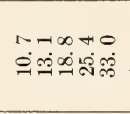 & 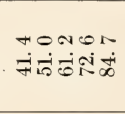 & 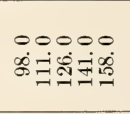 & 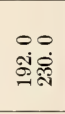 \\
\hline & & 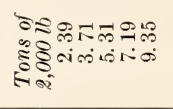 & 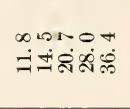 & 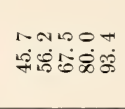 & 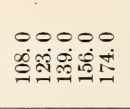 & $\begin{array}{l}00 \\
\text { तี่ } \\
\text { तें }\end{array}$ \\
\hline & \multirow{2}{*}{ 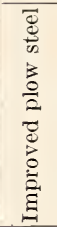 } & 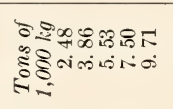 & 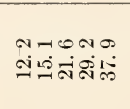 & 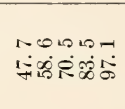 & 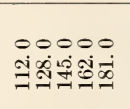 & 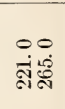 \\
\hline & & 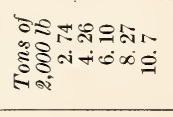 & 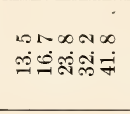 & 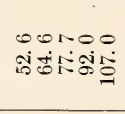 & 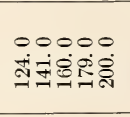 & 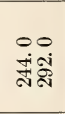 \\
\hline \multirow{2}{*}{\multicolumn{2}{|c|}{ 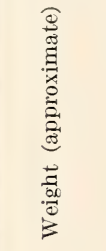 }} & 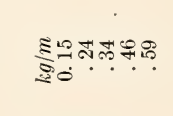 & 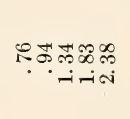 & 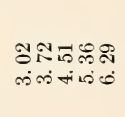 & 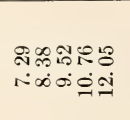 & $\begin{array}{l}\infty 8 \\
\infty 8 \\
+i \infty\end{array}$ \\
\hline & & 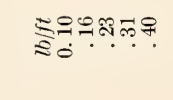 & எேஃะே & 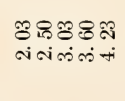 & 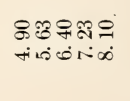 & $\begin{array}{l}8 \cong \\
\stackrel{-1}{=}\end{array}$ \\
\hline \multirow{2}{*}{\multicolumn{2}{|c|}{ 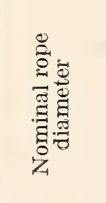 }} & 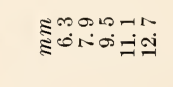 & 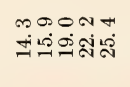 & 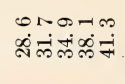 & 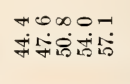 & $\begin{array}{l}n \infty \\
\dot{8} 80\end{array}$ \\
\hline & & Eే & 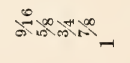 & 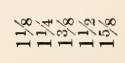 & 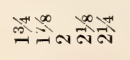 & 체 \\
\hline
\end{tabular}




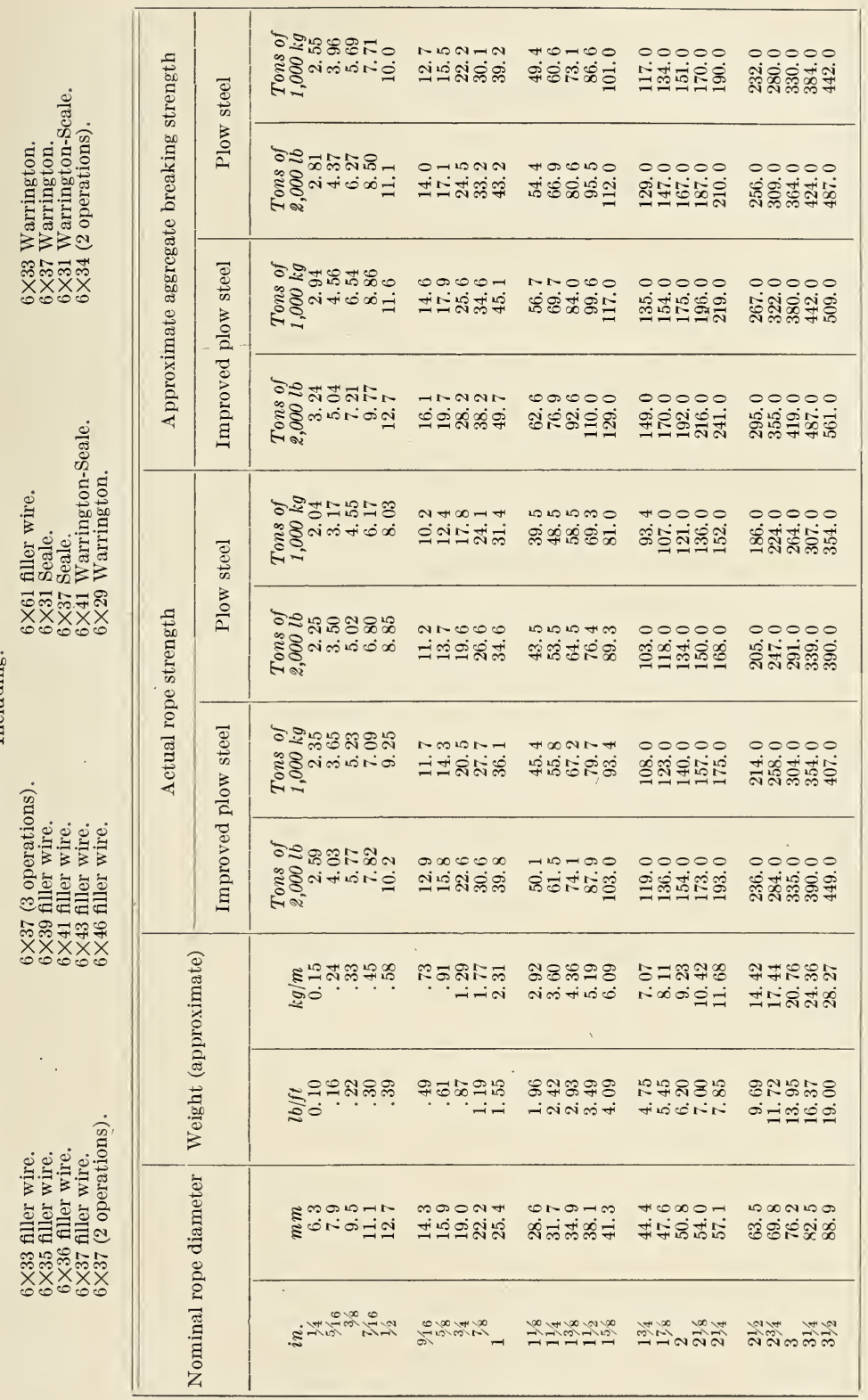




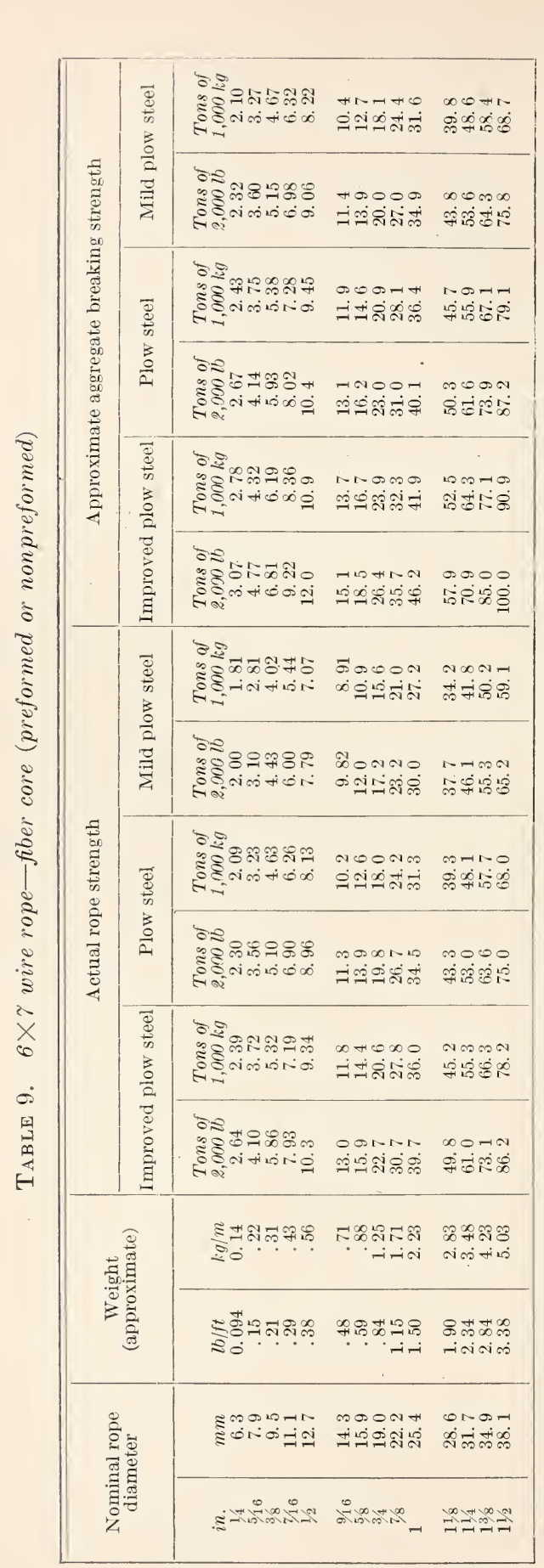


TABLE 10. $8 \times 19$ wire rope-fiber core (preformed or nonpreformed)

\section{Including:}

$8 \times 19$ Seale.

$8 \times 19$ Warrington.

$8 \times 25$ flller wire.

\begin{tabular}{|c|c|c|c|c|c|c|c|c|c|c|c|}
\hline \multirow{2}{*}{\multicolumn{2}{|c|}{$\begin{array}{l}\text { Nominal rope } \\
\text { diameter }\end{array}$}} & \multirow{2}{*}{\multicolumn{2}{|c|}{$\begin{array}{c}\text { Weight } \\
\text { (approximate) }\end{array}$}} & \multicolumn{4}{|c|}{ Actual rope strength } & \multicolumn{4}{|c|}{$\begin{array}{c}\text { Approximate aggregate breaking } \\
\text { strength }\end{array}$} \\
\hline & & & & \multicolumn{2}{|c|}{$\underset{\text { steel }}{\text { Improved plow }}$} & \multicolumn{2}{|c|}{ Plow steel } & \multicolumn{2}{|c|}{$\begin{array}{c}\text { Improved plow } \\
\text { steel }\end{array}$} & \multicolumn{2}{|c|}{ Plow steel } \\
\hline $\begin{array}{c}\text { in. } \\
1 / 4 \\
5 / 16 \\
3 / 8 \\
7 / 16 \\
1 / 2\end{array}$ & $\begin{array}{r}m m \\
6.3 \\
7.9 \\
9.5 \\
11.1 \\
12.7\end{array}$ & $\begin{array}{r}l b / f t \\
0.09 \\
.14 \\
.20 \\
.28 \\
.36\end{array}$ & $\begin{array}{r}\mathrm{kg} / \mathrm{m} \\
0.13 \\
.21 \\
.30 \\
.42 \\
.54\end{array}$ & $\begin{array}{c}\text { Tons of } \\
2,000 \mathrm{lb} \\
2.35 \\
3.65 \\
5.24 \\
7.09 \\
9.23\end{array}$ & $\begin{array}{c}\text { Tons of } \\
1,000 \mathrm{~kg} \\
2.13 \\
3.31 \\
4.75 \\
6.43 \\
8.37\end{array}$ & $\begin{array}{c}\text { Tons of } \\
2,000 \mathrm{lb} \\
2.04 \\
3.18 \\
4.55 \\
6.17 \\
8.02\end{array}$ & $\begin{array}{c}\text { Tons of } \\
1,000 \mathrm{~kg} \\
1.85 \\
2.88 \\
4.13 \\
5.60 \\
7.27\end{array}$ & $\begin{array}{c}\text { Tons of } \\
2,000 \mathrm{lb} \\
2.85 \\
4.42 \\
6.35 \\
8.59 \\
11.2\end{array}$ & $\begin{array}{c}\text { Tons of } \\
1,000 \mathrm{~kg} \\
2.58 \\
4.01 \\
5.76 \\
7.79 \\
10.1\end{array}$ & $\begin{array}{r}\text { Tons of } \\
2,000 \mathrm{lb} \\
2.47 \\
3.85 \\
5.51 \\
7.48 \\
9.72\end{array}$ & $\begin{array}{c}\text { Tons of } \\
1,000 \mathrm{~kg} \\
2.24 \\
3.49 \\
5.01 \\
6.79 \\
8.81\end{array}$ \\
\hline $\begin{array}{c}9 / 16 \\
5 / 8 \\
3 / 4 \\
1^{7 / 8}\end{array}$ & $\begin{array}{l}14.3 \\
15.9 \\
19.0 \\
22.2 \\
25.4\end{array}$ & $\begin{array}{r}.46 \\
.57 \\
.82 \\
1.11 \\
1.45\end{array}$ & $\begin{array}{r}.68 \\
.85 \\
1.22 \\
1.65 \\
2.16\end{array}$ & $\begin{array}{l}11.6 \\
14.3 \\
20.5 \\
27.7 \\
36.0\end{array}$ & $\begin{array}{l}10.5 \\
13.0 \\
18.6 \\
25.1 \\
32.6\end{array}$ & $\begin{array}{l}10.1 \\
12.4 \\
17.8 \\
24.1 \\
31.3\end{array}$ & $\begin{array}{l}9.16 \\
11.2 \\
16.1 \\
21.9 \\
28.4\end{array}$ & $\begin{array}{l}14.1 \\
17.3 \\
24.8 \\
33.6 \\
43.6\end{array}$ & $\begin{array}{l}12.7 \\
15.7 \\
22.5 \\
30.4 \\
39.5\end{array}$ & $\begin{array}{l}12.2 \\
15.0 \\
21.6 \\
29.2 \\
37.9\end{array}$ & $\begin{array}{l}11.1 \\
13.6 \\
19.5 \\
26.5 \\
34.4\end{array}$ \\
\hline $\begin{array}{l}11 / 8 \\
11 / 4 \\
13 / 8 \\
11 / 2\end{array}$ & $\begin{array}{l}28.6 \\
31.7 \\
34.9 \\
38.1\end{array}$ & $\begin{array}{l}1.84 \\
2.27 \\
2.74 \\
3.26\end{array}$ & $\begin{array}{l}2.74 \\
3.38 \\
4.08 \\
4.85\end{array}$ & $\begin{array}{l}45.3 \\
55.7 \\
67.1 \\
79.4\end{array}$ & $\begin{array}{l}41.1 \\
50.5 \\
60.9 \\
72.0\end{array}$ & $\begin{array}{l}39.4 \\
48.4 \\
58.3 \\
69.1\end{array}$ & $\begin{array}{l}35.7 \\
43.9 \\
52.9 \\
62.7\end{array}$ & $\begin{array}{l}54.9 \\
67.5 \\
81.3 \\
96.2\end{array}$ & $\begin{array}{l}49.8 \\
61.2 \\
73.8 \\
87.3\end{array}$ & $\begin{array}{l}47.7 \\
58.7 \\
70.7 \\
83.7\end{array}$ & $\begin{array}{l}43.3 \\
53.2 \\
64.1 \\
76.0\end{array}$ \\
\hline
\end{tabular}

NOTE.-For breaking strengths of galvanized ropes, deduct $10 \%$ from strengths shown.

TABLE 11. Flattened strand ropes $-6 \times 25$ type $B ; 6 \times 30$ type $G$

\begin{tabular}{|c|c|c|c|c|c|c|c|c|c|c|c|}
\hline \multirow{2}{*}{\multicolumn{2}{|c|}{$\begin{array}{l}\text { Nominal rope } \\
\text { diameter }\end{array}$}} & \multirow{2}{*}{\multicolumn{2}{|c|}{$\begin{array}{c}\text { Weight } \\
\text { (approximate) }\end{array}$}} & \multicolumn{4}{|c|}{ Actual rope strength } & \multicolumn{4}{|c|}{$\begin{array}{c}\text { Approximate agglegate breaking } \\
\text { strength }\end{array}$} \\
\hline & & & & \multicolumn{2}{|c|}{$\begin{array}{l}\text { Improved plow } \\
\text { steel }\end{array}$} & \multicolumn{2}{|c|}{ Mild plow steel } & \multicolumn{2}{|c|}{$\begin{array}{l}\text { Improved plow } \\
\text { steel }\end{array}$} & \multicolumn{2}{|c|}{ Mild plow steel } \\
\hline in. & $m m$ & $l b / f t$ & $\mathrm{~kg} / \mathrm{m}$ & $\begin{array}{l}\text { Tons of } \\
2,000 \mathrm{lb}\end{array}$ & $\begin{array}{ll}\text { Tons } & \text { of } \\
1,000 & \mathrm{~kg}\end{array}$ & $\begin{array}{l}\text { Tons of } \\
2,000 \mathrm{lb}\end{array}$ & $\begin{array}{l}\text { Tons of } \\
1,000 \mathrm{~kg}\end{array}$ & $\begin{array}{l}\text { Tons of } \\
2,000 \mathrm{lb}\end{array}$ & $\begin{array}{ll}\text { Tors } & \text { of } \\
1,000 & \mathrm{~kg}\end{array}$ & $\begin{array}{l}\text { Tons of } \\
2,000 \mathrm{lb}\end{array}$ & $\begin{array}{l}\text { Tons of } \\
1,000 \quad \mathrm{~kg}\end{array}$ \\
\hline $3 / 8$ & 9.5 & 0.25 & 0.37 & 6. 71 & 6.09 & - & . & $\begin{array}{r}7.89 \\
\end{array}$ & 7.16 & $\begin{array}{c}n,-\infty \\
-\ldots\end{array}$ & 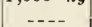 \\
\hline $1 / 2$ & 12. 7 & .45 & .67 & 11.8 & 10.7 & 8.94 & 8.11 & 13.9 & 12.6 & 10.5 & 9.54 \\
\hline $9 / 16$ & 14.3 & .57 & .85 & 14.9 & 13.5 & 11.2 & 10.2 & 17.5 & 15.9 & 13.2 & 12.0 \\
\hline $5 / 8$ & 15.9 & .70 & 1.04 & 18.3 & 16.6 & 13.9 & 12.6 & 21.5 & 19.5 & 16.3 & 14.8 \\
\hline $3 / 4$ & 19.0 & 1.01 & 1.50 & 26.2 & 23.8 & 19.8 & 18.0 & 30.8 & 28.0 & 23.3 & 21.2 \\
\hline $7 / 8$ & 22.2 & 1.39 & 2.07 & 35.4 & 32.1 & 26.8 & 24.3 & 41.6 & 37.8 & 31.5 & 28.6 \\
\hline 1 & 25.4 & 1. 80 & 2. 68 & 46.0 & 41.7 & 34.8 & 31.6 & 54.1 & 49.0 & 40.9 & 37.2 \\
\hline $11 / 8$ & 28.6 & 2. 28 & 3.39 & 57.9 & 52.5 & 43.8 & 39.7 & 68.1 & 61.8 & 51.5 & 46.7 \\
\hline $11 / 4$ & 31.7 & 2.81 & 4. 18 & 71.0 & 64.4 & 53.7 & 48.7 & 83.5 & 75.8 & 63.2 & 57.3 \\
\hline $13 / 8$ & 34.9 & 3.40 & 5.06 & 85.5 & 77.6 & --- & $\ldots$ & 100.0 & 91.3 & $\ldots$ & -.. \\
\hline $11 / 2$ & 38.1 & 4.05 & 6.03 & 101.0 & 91.6 & & & 119.0 & 108.0 & & \\
\hline $15 \%$ & 41.3 & 4. 75 & 7.07 & 118.0 & 107.0 & - & - . & 139.0 & 126.0 & - - - & - . - \\
\hline $13 / 4$ & 44. 4 & 5.51 & 8.20 & 136.0 & 123.0 & $\ldots$ & -... & 160.0 & 145.0 & -- & -- \\
\hline
\end{tabular}

Note.-For wire strand cores and independent wire rope cores, add $7 \frac{1}{2} \%$ to the listed strengths and $5 \%$ to the weights. 
TABLE 12. Flattened strand rope-6×8

\begin{tabular}{|c|c|c|c|c|c|c|c|c|c|c|c|}
\hline \multirow{2}{*}{\multicolumn{2}{|c|}{$\begin{array}{l}\text { Nominal rope } \\
\text { diameter }\end{array}$}} & \multirow{2}{*}{\multicolumn{2}{|c|}{$\begin{array}{c}\text { Weight } \\
\text { (approximate) }\end{array}$}} & \multicolumn{4}{|c|}{ Actual rope strength } & \multicolumn{4}{|c|}{$\begin{array}{c}\text { Approximate aggregate breaking } \\
\text { strength }\end{array}$} \\
\hline & & & & \multicolumn{2}{|c|}{$\begin{array}{l}\text { Improved plow } \\
\text { steel }\end{array}$} & \multicolumn{2}{|c|}{ Mild plow steel } & \multicolumn{2}{|c|}{$\begin{array}{c}\text { Improved plow } \\
\text { steel }\end{array}$} & \multicolumn{2}{|c|}{ Mild plow steel } \\
\hline & & lh/ft & $\mathrm{l} a / m$ & Tons of & Tons of & Tons of & Tons of & Tons of & Tons of & Tons of & Tons of \\
\hline $\begin{array}{l}m_{1 / 2} \\
1\end{array}$ & 12.7 & $\begin{array}{l}l 0 / \int l \\
0.45\end{array}$ & $\begin{array}{l}\kappa g / m \\
0.67\end{array}$ & $\begin{array}{l}2,000 \\
11.1\end{array}$ & $\begin{array}{c}1,000.1 \\
10.1\end{array}$ & $\begin{array}{r}2,000 \\
8.37\end{array}$ & $\begin{array}{c}1,000 \mathrm{~kg} \\
7.59\end{array}$ & $\begin{array}{l}2,00000 \\
12.5\end{array}$ & $\begin{array}{l}11.4 \\
11.4\end{array}$ & $\begin{array}{r}2,00010 \\
9.46\end{array}$ & $\begin{array}{l}1,000 \mathrm{~kg} \\
8.58\end{array}$ \\
\hline $5 \%$ & 15.9 & .70 & 1.04 & 17. 1 & 15.5 & 12.9 & 11.7 & 19.3 & 17.5 & 14.6 & 13.2 \\
\hline 34 & 19.0 & 1.01 & 1.50 & 24.4 & 22.1 & 18.5 & 16.8 & 27.6 & 25.0 & 20.9 & 19.0 \\
\hline $7 / 8$ & 22.2 & 1.39 & 2.07 & 33.0 & 29.9 & 24.9 & 22.6 & 37.3 & 33.8 & 28.1 & 25.5 \\
\hline $1^{/ 0}$ & 25.4 & 1.80 & 2.68 & 42.7 & 38.7 & 32.3 & 29.3 & 48. 2 & 43.7 & 36.5 & 33.1 \\
\hline $11 / 8$ & 28.6 & 2. 28 & 3.39 & 53.5 & 48.5 & 40.5 & 36.7 & 60.4 & 54.8 & 45.8 & 41.5 \\
\hline $11 / 4$ & 31.7 & 2. 81 & 4.18 & 65.5 & 59.4 & 49.4 & 44.8 & 74.0 & 67.1 & 55.8 & 50.6 \\
\hline $13 \%$ & 34.9 & 3. 40 & 5.06 & 78.6 & 71.3 & 59.4 & 53.9 & 88.8 & 80.6 & 67.1 & 60.9 \\
\hline $11 / 2$ & 38.1 & 4.05 & 6.03 & 92.7 & 84.1 & 70.1 & 63.6 & $\therefore 05.0$ & 95.0 & 79.2 & 71.9 \\
\hline
\end{tabular}

Note.-For wire strand cores and independent wire rope cores add $71 / 2 \%$ to the listed strengths and $5 \%$ to the weights.

TABLE 13. Galvanized highway-guard cable

\begin{tabular}{|c|c|c|c|c|c|c|c|c|}
\hline \multicolumn{2}{|c|}{$\begin{array}{l}\text { Nominal cable } \\
\text { diameter }\end{array}$} & \multirow{2}{*}{$\begin{array}{c}\begin{array}{c}\text { Construc- } \\
\text { tion }\end{array} \\
\\
\\
3 \times 7 \\
7 \times 7\end{array}$} & \multicolumn{2}{|c|}{$\begin{array}{c}\text { Weight } \\
\text { (approximate) }\end{array}$} & \multicolumn{2}{|c|}{ Actual cable strength } & \multicolumn{2}{|c|}{$\begin{array}{l}\text { Approximate aggre- } \\
\text { gate breaking strength }\end{array}$} \\
\hline $1^{i, 4}$ & $\begin{array}{l}m m \\
19.0 \\
25.4\end{array}$ & & $\begin{array}{l}l b / f t \\
0.85 \\
1.6\end{array}$ & $\begin{array}{l}\mathrm{kg} / \mathrm{m} \\
1.26 \\
2.38\end{array}$ & $\begin{array}{l}\text { Tons of } \\
2,000 \mathrm{lb} \\
12.5 \\
15.0\end{array}$ & $\begin{array}{l}\text { Tons of } \\
1,000 \mathrm{~kg} \\
11.3 \\
13.6\end{array}$ & $\begin{array}{l}\text { Tons of } \\
2,000 l b \\
13.9 \\
17.4\end{array}$ & $\begin{array}{c}\text { Tons of } \\
1,000 \mathrm{~kg} \\
12.5 \\
15.8\end{array}$ \\
\hline
\end{tabular}




\begin{tabular}{|c|c|c|c|c|c|c|}
\hline \multirow{4}{*}{ 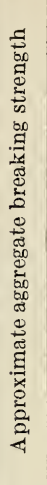 } & \multirow{2}{*}{ 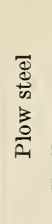 } & 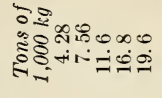 & 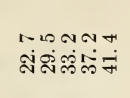 & M-ON= & 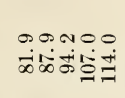 & $\stackrel{\circ}{\stackrel{\Phi}{-1}}$ \\
\hline & & 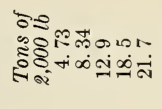 & 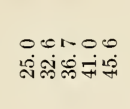 & 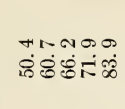 & $\begin{array}{l}\text { mo00 } \\
\dot{8} \dot{8}+\dot{0} \cong \dot{\Xi}\end{array}$ & 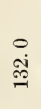 \\
\hline & \multirow{2}{*}{ 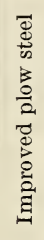 } & 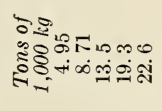 & 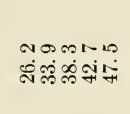 & 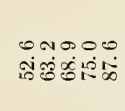 & 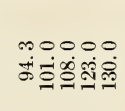 & $\stackrel{\circ}{\stackrel{\infty}{\oplus}}$ \\
\hline & & 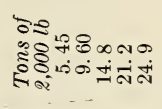 & 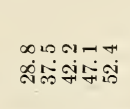 & 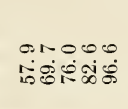 & 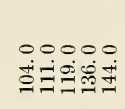 & $\begin{array}{l}\circ \\
\text { : } \\
\stackrel{0}{:}\end{array}$ \\
\hline \multirow{4}{*}{ 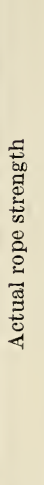 } & \multirow{2}{*}{ 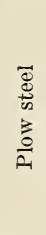 } & 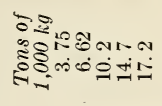 & 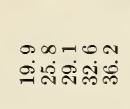 & 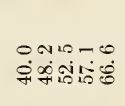 & 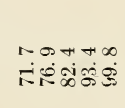 & $\begin{array}{l}0 \\
i \dot{8} \\
\stackrel{=}{=}\end{array}$ \\
\hline & & 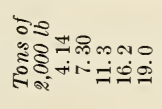 & 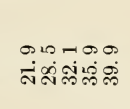 & 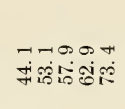 & $\begin{array}{l}0 \infty \infty 00 \\
\infty+\infty \dot{S} \leftrightarrow \dot{\Theta}\end{array}$ & $\begin{array}{l}\stackrel{\theta}{\Xi} \\
\end{array}$ \\
\hline & \multirow{2}{*}{ 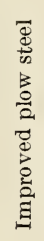 } & 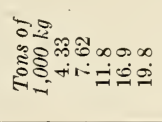 & 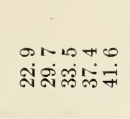 & 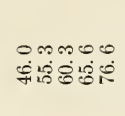 & 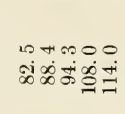 & $\stackrel{\circ}{\dot{I}}$ \\
\hline & & 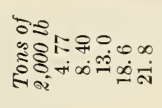 & 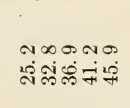 & 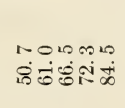 & 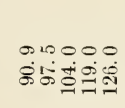 & $\stackrel{\circ}{\stackrel{0}{9}}$ \\
\hline \multirow{2}{*}{\multicolumn{2}{|c|}{ 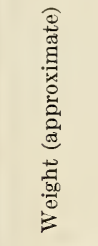 }} & 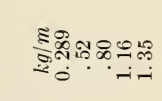 & 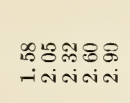 & 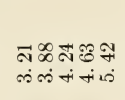 & 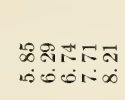 & 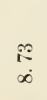 \\
\hline & & 뇨 & 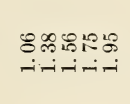 & 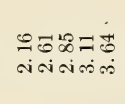 & 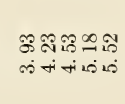 & $\begin{array}{l}1 \\
\infty \\
\text { is }\end{array}$ \\
\hline \multirow{2}{*}{\multicolumn{2}{|c|}{ 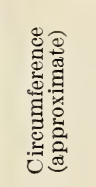 }} & 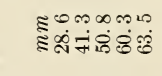 & 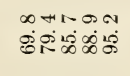 & 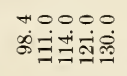 & 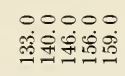 & $\begin{array}{l}0 \\
\dot{8} \\
\stackrel{8}{6}\end{array}$ \\
\hline & & 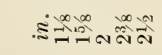 & 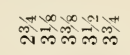 & 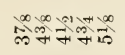 & 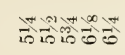 & $\stackrel{30}{6}$ \\
\hline \multirow{2}{*}{\multicolumn{2}{|c|}{ 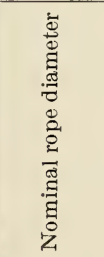 }} & 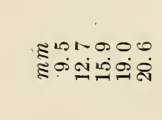 & 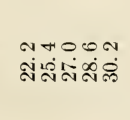 & 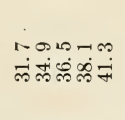 & 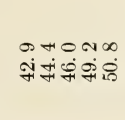 & 공 \\
\hline & & 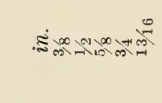 & 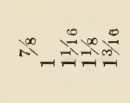 & $=0 \times-\infty$ & 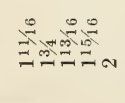 & $\stackrel{0}{\sim}$ \\
\hline
\end{tabular}




\begin{tabular}{|c|c|c|c|c|c|c|}
\hline \multirow{4}{*}{ 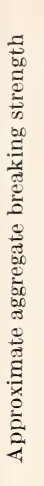 } & \multirow{2}{*}{ 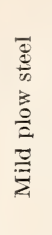 } & 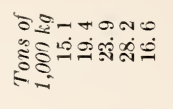 & 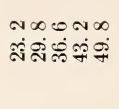 & 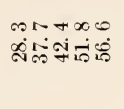 & 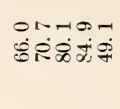 & 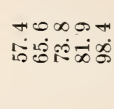 \\
\hline & & 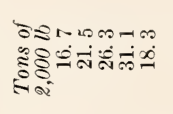 & 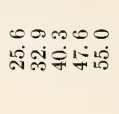 & 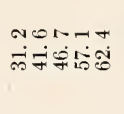 & 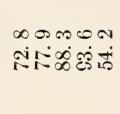 & 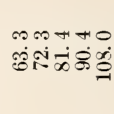 \\
\hline & \multirow{2}{*}{$\begin{array}{l}\bar{d} \\
\frac{d}{n} \\
\frac{3}{0} \\
\frac{3}{2}\end{array}$} & 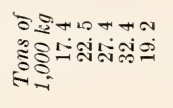 & 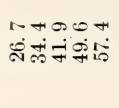 & 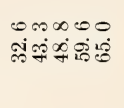 & 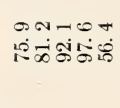 & 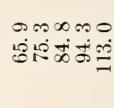 \\
\hline & & 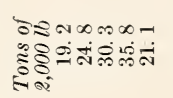 & 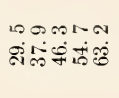 & 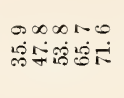 & 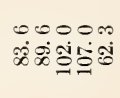 & 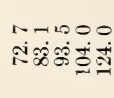 \\
\hline \multirow{4}{*}{ 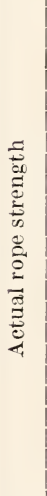 } & \multirow{2}{*}{ 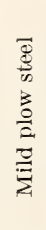 } & 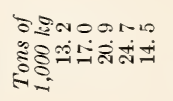 & 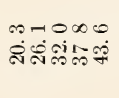 & 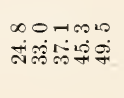 & 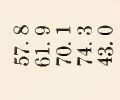 & 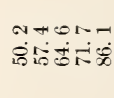 \\
\hline & & 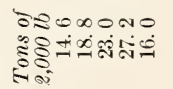 & 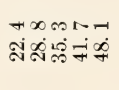 & 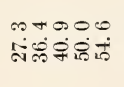 & 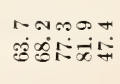 & 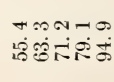 \\
\hline & \multirow{2}{*}{ 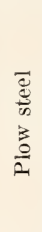 } & 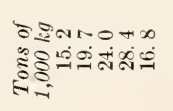 & هิำ & 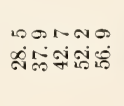 & 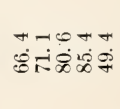 & 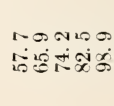 \\
\hline & & 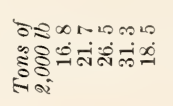 & 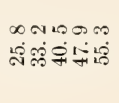 & 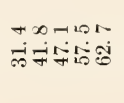 & 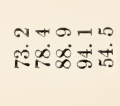 & 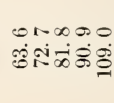 \\
\hline \multirow{2}{*}{\multicolumn{2}{|c|}{ 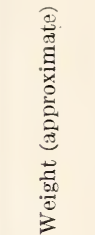 }} & ह8のה & 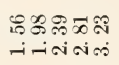 & 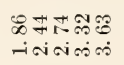 & 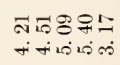 & 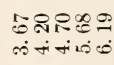 \\
\hline & & 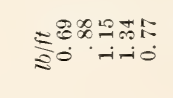 & 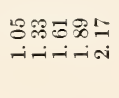 & 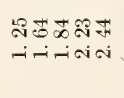 & 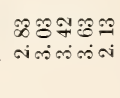 & 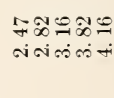 \\
\hline \multirow{2}{*}{\multicolumn{2}{|c|}{ 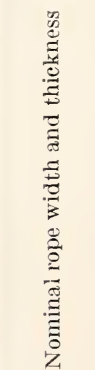 }} & 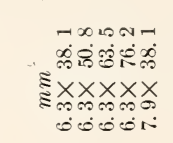 & 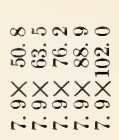 & 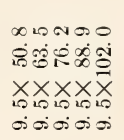 & 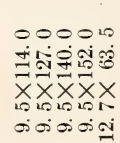 & 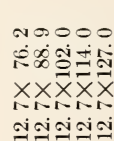 \\
\hline & & 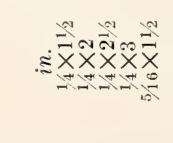 & 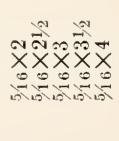 & 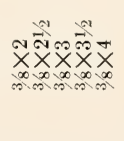 & 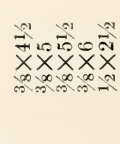 & 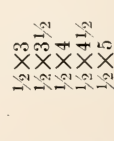 \\
\hline
\end{tabular}


000tm 00000000000000

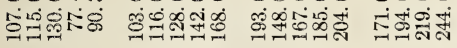

000Nis 00000000000000

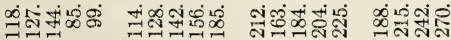

0000000000000000000

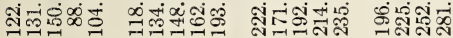

0000000000000000000

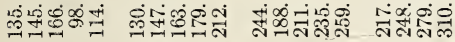

HoONo m0000 000000000

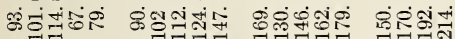

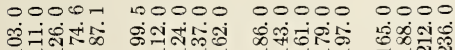

00001 00000 00000 0000

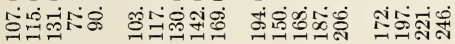

0000000000000000000

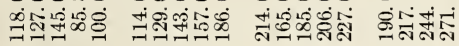

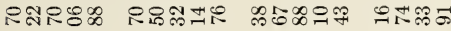

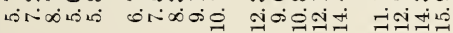

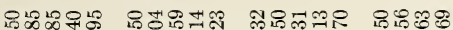

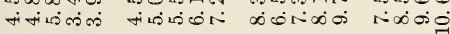
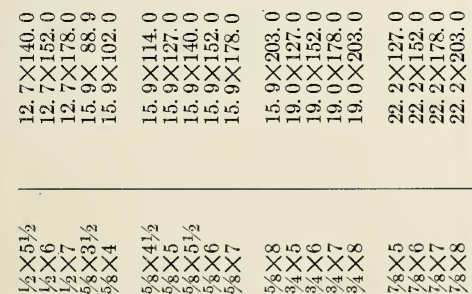
TABLE $16.18 \times 7$ nonrotating wire rope

\begin{tabular}{|c|c|c|c|c|c|c|c|c|c|c|c|}
\hline \multirow{2}{*}{\multicolumn{2}{|c|}{$\begin{array}{l}\text { Nominal rope } \\
\text { diameter }\end{array}$}} & \multirow{2}{*}{\multicolumn{2}{|c|}{$\begin{array}{l}\text { Weight (ap- } \\
\text { proximate) }\end{array}$}} & \multicolumn{4}{|c|}{ Actual rope strength } & \multicolumn{4}{|c|}{$\begin{array}{c}\text { Approximate aggregate breaking } \\
\text { strength }\end{array}$} \\
\hline & & & & \multicolumn{2}{|c|}{$\begin{array}{l}\text { Improved plow } \\
\text { steel }\end{array}$} & \multicolumn{2}{|c|}{ Plow steel } & \multicolumn{2}{|c|}{$\begin{array}{l}\text { Improved plow } \\
\text { steel }\end{array}$} & \multicolumn{2}{|c|}{ Plow steel } \\
\hline $\begin{array}{r}\text { in. } \\
3 / 8 \\
7 / 16 \\
1 / 2 \\
9 / 16 \\
5 / 8\end{array}$ & $\begin{array}{r}m m \\
9.5 \\
11.1 \\
12.7 \\
14.3 \\
15.9\end{array}$ & $\begin{array}{r}l b / f t \\
0.24 \\
.33 \\
.43 \\
.55 \\
.68\end{array}$ & $\begin{array}{r}\mathrm{kg} / \mathrm{m} \\
0.36 \\
.49 \\
.64 \\
.82 \\
1.01\end{array}$ & $\begin{array}{c}\text { Tons of } \\
2,000 \text { lb } \\
5.59 \\
7.58 \\
9.85 \\
12.4 \\
15.3\end{array}$ & $\begin{array}{c}\text { Tons of } \\
1,000 \mathrm{~kg} \\
5.07 \\
6.88 \\
8.93 \\
11.2 \\
13.9\end{array}$ & $\begin{array}{c}\text { Tens of } \\
2,000 \mathrm{lb} \\
4.86 \\
6.59 \\
8.57 \\
10.8 \\
13.3\end{array}$ & $\begin{array}{c}\text { Tons of } \\
1,000 \mathrm{~kg} \\
4.41 \\
5.98 \\
7.77 \\
9.80 \\
12.1\end{array}$ & $\begin{array}{c}\text { Tons of } \\
2,000 \mathrm{lb} \\
7.76 \\
10.5 \\
13.7 \\
17.2 \\
21.2\end{array}$ & $\begin{array}{c}\text { Tons of } \\
1,000 \mathrm{~kg} \\
7.04 \\
9.55 \\
12.4 \\
15.5 \\
19.3\end{array}$ & $\begin{array}{c}\text { Tons of } \\
2,000 \mathrm{lb} \\
6.75 \\
9.15 \\
11.9 \\
15.0 \\
18.5\end{array}$ & $\begin{array}{c}\text { Tons of } \\
1,000 \mathrm{~kJ} \\
6.12 \\
8.39 \\
10.8 \\
13.6 \\
16.8\end{array}$ \\
\hline $\begin{array}{l}3 / 4 \\
7 / 8 \\
1 \\
11 / 8 \\
11 / 4\end{array}$ & $\begin{array}{l}19.0 \\
22.2 \\
25.4 \\
28.6 \\
31.7\end{array}$ & $\begin{array}{r}.97 \\
1.32 \\
1.73 \\
2.19 \\
2.70\end{array}$ & $\begin{array}{l}1.44 \\
1.96 \\
2.57 \\
3.26 \\
4.02\end{array}$ & $\begin{array}{l}21.8 \\
29.5 \\
38.3 \\
48.2 \\
59.2\end{array}$ & $\begin{array}{l}19.8 \\
26.8 \\
34.7 \\
43.7 \\
53.7\end{array}$ & $\begin{array}{l}19.0 \\
25.7 \\
33.3 \\
41.9 \\
51.5\end{array}$ & $\begin{array}{l}17.2 \\
23.3 \\
30.2 \\
38.0 \\
46.7\end{array}$ & $\begin{array}{l}30.3 \\
41.0 \\
53.2 \\
66.9 \\
82.2\end{array}$ & $\begin{array}{l}27.5 \\
37.2 \\
48.2 \\
60.7 \\
74.6\end{array}$ & $\begin{array}{l}26.4 \\
35.7 \\
46.2 \\
58.2 \\
71.5\end{array}$ & $\begin{array}{l}23.9 \\
32.4 \\
41.9 \\
52.8 \\
64.9\end{array}$ \\
\hline $\begin{array}{l}13 / 8 \\
11 / 2 \\
15 / 8 \\
13 / 4\end{array}$ & $\begin{array}{l}34.9 \\
38.1 \\
41.3 \\
44.4\end{array}$ & $\begin{array}{l}\text { 3. } 27 \\
\text { 3. } 89 \\
4.57 \\
5.30\end{array}$ & $\begin{array}{l}4.87 \\
5.79 \\
6.80 \\
7.89\end{array}$ & $\begin{array}{r}71.3 \\
84.4 \\
98.4 \\
114.0\end{array}$ & $\begin{array}{r}64.7 \\
76.6 \\
89.3 \\
103.0\end{array}$ & $\begin{array}{l}62.0 \\
73.4 \\
85.6 \\
98.8\end{array}$ & $\begin{array}{l}56.2 \\
66.6 \\
77.6 \\
89.6\end{array}$ & $\begin{array}{r}99.0 \\
117.0 \\
137.0 \\
158.0\end{array}$ & $\begin{array}{r}89.9 \\
106.0 \\
124.0 \\
143.0\end{array}$ & $\begin{array}{r}86.1 \\
102.0 \\
119.0 \\
137.0\end{array}$ & $\begin{array}{r}78.0 \\
92.5 \\
108.0 \\
124.0\end{array}$ \\
\hline
\end{tabular}

TABLE $17.6 \times 42$ tiller rope

\begin{tabular}{|c|c|c|c|c|c|c|c|c|c|c|c|}
\hline \multirow{2}{*}{\multicolumn{2}{|c|}{$\begin{array}{l}\text { Nominal rope } \\
\text { diameter }\end{array}$}} & \multirow{2}{*}{\multicolumn{2}{|c|}{$\begin{array}{l}\text { Weight (ap- } \\
\text { proximate) }\end{array}$}} & \multicolumn{4}{|c|}{ Actual rope strength } & \multicolumn{4}{|c|}{$\begin{array}{c}\text { Approximate aggregate breaking } \\
\text { strength }\end{array}$} \\
\hline & & & & \multicolumn{2}{|c|}{ Plow steel } & \multicolumn{2}{|c|}{ Iron } & \multicolumn{2}{|c|}{ Plow steel } & \multicolumn{2}{|c|}{ Iron } \\
\hline in. $\begin{array}{r}1 / 4 \\
5 / 16 \\
3 / 8 \\
7 / 16 \\
1 / 2\end{array}$ & $\begin{array}{r}m m \\
6.3 \\
7.9 \\
9.5 \\
11.1 \\
12.7\end{array}$ & $\begin{array}{c}l b / f t \\
0.07 \\
.11 \\
.16 \\
.21 \\
.28\end{array}$ & $\begin{array}{c}\mathrm{kg} / \mathrm{m} \\
0.10 \\
.16 \\
.24 \\
.31 \\
.42\end{array}$ & $\begin{array}{c}\text { Tons of } \\
2,000 \mathrm{lb} \\
1.31 \\
2.05 \\
2.93 \\
3.98 \\
5.18\end{array}$ & $\begin{array}{r}\text { Tons of } \\
1,000 \mathrm{~kg} \\
1.19 \\
1.86 \\
2.66 \\
3.61 \\
4.70\end{array}$ & $\begin{array}{c}\text { Tons of } \\
2,000 \mathrm{lb} \\
0.584 \\
.908 \\
1.30 \\
1.77 \\
2.30\end{array}$ & $\begin{array}{r}\text { Tons of } \\
1,000 \mathrm{~kg} \\
0.53 \\
.82 \\
1.18 \\
1.60 \\
2.09\end{array}$ & $\begin{array}{c}\text { Tons of } \\
2,000 \mathrm{lb} \\
1.82 \\
2.84 \\
4.07 \\
5.50 \\
7.195\end{array}$ & $\begin{array}{r}\text { Tons of } \\
1,000 \mathrm{~kg} \\
1.65 \\
2.58 \\
3.69 \\
5.01 \\
6.53\end{array}$ & $\begin{array}{c}\text { Tons of } \\
2,0001 \mathrm{lb} \\
0.810 \\
1.260 \\
1.805 \\
2.460 \\
3.195\end{array}$ & $\begin{array}{c}\text { Tons of } \\
1,000 \mathrm{~kg} \\
0.736 \\
1.140 \\
1.640 \\
2.220 \\
2.900\end{array}$ \\
\hline $\begin{array}{r}9 / 16 \\
5 / 8\end{array}$ & $\begin{array}{l}14.3 \\
15.9\end{array}$ & $\begin{array}{l}.35 \\
.43\end{array}$ & $\begin{array}{l}.52 \\
.64\end{array}$ & $\begin{array}{l}6.53 \\
8.04\end{array}$ & $\begin{array}{l}5.92 \\
7.29\end{array}$ & $\begin{array}{l}2.90 \\
3.57\end{array}$ & $\begin{array}{l}2.63 \\
3.24\end{array}$ & $\begin{array}{r}9.07 \\
11.16\end{array}$ & $\begin{array}{r}8.22 \\
10.12\end{array}$ & $\begin{array}{l}4.025 \\
4.960\end{array}$ & $\begin{array}{l}3.650 \\
4.500\end{array}$ \\
\hline
\end{tabular}


TABLE 18. Locked coil track strand

\begin{tabular}{|c|c|c|c|c|c|c|c|c|c|c|c|}
\hline \multirow{2}{*}{\multicolumn{2}{|c|}{$\begin{array}{c}\text { Nominal strand } \\
\text { diameter }\end{array}$}} & \multirow{2}{*}{\multicolumn{2}{|c|}{$\begin{array}{l}\text { Weight (ap- } \\
\text { proximate) }\end{array}$}} & \multicolumn{4}{|c|}{ Actual strand strength } & \multicolumn{4}{|c|}{$\begin{array}{c}\text { Approximate aggregate breaking } \\
\text { strength }\end{array}$} \\
\hline & & & & \multicolumn{2}{|c|}{ Special grade } & \multicolumn{2}{|c|}{ Standard grade } & \multicolumn{2}{|c|}{ Special grade } & \multicolumn{2}{|c|}{ Standard grade } \\
\hline $\begin{array}{l}\text { in. } \\
3 / 4 \\
7 / 8 \\
1 \\
11 / 8 \\
11 / 4\end{array}$ & $\begin{array}{l}m m \\
19.0 \\
22.2 \\
25.4 \\
28.6 \\
31.7\end{array}$ & $\begin{array}{c}l b / f t \\
1.41 \\
1.92 \\
2.50 \\
3.16 \\
3.91\end{array}$ & $\begin{array}{c}\mathrm{kg} / \mathrm{m} \\
2.10 \\
2.86 \\
3.72 \\
4.70 \\
5.82\end{array}$ & $\begin{array}{c}\text { Tons of } \\
2,000 l \bar{l} \\
31.5 \\
41.5 \\
52.5 \\
66.0 \\
81.0\end{array}$ & $\begin{array}{r}\text { Tons of } \\
1,000 \mathrm{~kg} \\
28.6 \\
37.6 \\
47.6 \\
59.9 \\
73.5\end{array}$ & $\begin{array}{r}\text { Tons of } \\
2,000 \text { lb } \\
25.0 \\
32.0 \\
42.0 \\
54.0 \\
65.0\end{array}$ & $\begin{array}{r}\text { Tons of } \\
1,000 \mathrm{~kg} \\
22.7 \\
29.0 \\
38.1 \\
49.0 \\
59.0\end{array}$ & $\begin{array}{c}\text { Tcns of } \\
2,000 \mathrm{lb} \\
35.0 \\
46.1 \\
58.3 \\
73.3 \\
90.0\end{array}$ & $\begin{array}{r}\text { Tons of } \\
1,000 \mathrm{~kg} \\
31.8 \\
41.8 \\
52.9 \\
66.5 \\
81.7\end{array}$ & $\begin{array}{r}\text { Tons of } \\
2,000 \text { lb } \\
27.8 \\
35.5 \\
46.7 \\
60.0 \\
72.2\end{array}$ & $\begin{array}{c}\text { Tons of } \\
1,000 \mathrm{~kg} \\
25.2 \\
32.2 \\
42.3 \\
54.4 \\
65.5\end{array}$ \\
\hline $\begin{array}{l}13 / 8 \\
11 / 2 \\
15 / 8 \\
13 / 4 \\
17 / 8\end{array}$ & $\begin{array}{l}34.9 \\
38.1 \\
41.3 \\
44.4 \\
47.6\end{array}$ & $\begin{array}{l}4.73 \\
5.63 \\
6.60 \\
7.66 \\
8.79\end{array}$ & $\begin{array}{r}7.04 \\
8.38 \\
9.82 \\
11.40 \\
13.08\end{array}$ & $\begin{array}{l}100.0 \\
120.5 \\
140.0 \\
165.0 \\
187.5\end{array}$ & $\begin{array}{r}90.7 \\
109.3 \\
127.0 \\
149.7 \\
170.1\end{array}$ & $\begin{array}{r}78.0 \\
93.0 \\
108.0 \\
125.0 \\
138.0\end{array}$ & $\begin{array}{r}70.8 \\
84.4 \\
98.0 \\
113.4 \\
125.2\end{array}$ & $\begin{array}{l}111.1 \\
133.9 \\
155.5 \\
183.3 \\
208.3\end{array}$ & $\begin{array}{l}100.8 \\
121.4 \\
141.1 \\
166.3 \\
189.0\end{array}$ & $\begin{array}{r}86.7 \\
103.3 \\
120.0 \\
138.9 \\
153.3\end{array}$ & $\begin{array}{r}78.7 \\
93.8 \\
108.9 \\
126.0 \\
139.1\end{array}$ \\
\hline $\begin{array}{l}2 \\
21 / 4 \\
21 / 2 \\
23 / 4 \\
3\end{array}$ & $\begin{array}{l}50.8 \\
57.1 \\
63.5 \\
69.8 \\
76.2\end{array}$ & $\begin{array}{l}10.00 \\
12.50 \\
15.20 \\
18.30 \\
22.20\end{array}$ & $\begin{array}{l}14.88 \\
18.60 \\
22.62 \\
27.23 \\
33.04\end{array}$ & $\begin{array}{l}215.0 \\
280.0 \\
345.0 \\
420.0 \\
500.0\end{array}$ & $\begin{array}{l}195.0 \\
254.0 \\
313.0 \\
381.0 \\
453.6\end{array}$ & 158.0 & 143.3 & $\begin{array}{l}238.9 \\
311.1 \\
383.3 \\
466.7 \\
555.5\end{array}$ & $\begin{array}{l}216.7 \\
282.2 \\
347.8 \\
423.3 \\
504.0\end{array}$ & 175.5 & 159.2 \\
\hline
\end{tabular}

TABLE 19. Smooth coil track strand

\begin{tabular}{|c|c|c|c|c|c|c|c|c|c|c|c|}
\hline \multirow{2}{*}{\multicolumn{2}{|c|}{$\begin{array}{l}\text { Nominal strand } \\
\text { diameter }\end{array}$}} & \multirow{2}{*}{\multicolumn{2}{|c|}{$\begin{array}{l}\text { Weight (ap- } \\
\text { proximate) }\end{array}$}} & \multicolumn{4}{|c|}{ Actual strand strength } & \multicolumn{4}{|c|}{$\begin{array}{c}\text { A pproximate aggregate breaking } \\
\text { strength }\end{array}$} \\
\hline & & & & \multicolumn{2}{|c|}{$\begin{array}{l}\text { Extra-high- } \\
\text { strength grade }\end{array}$} & \multicolumn{2}{|c|}{$\begin{array}{l}\text { High-strength } \\
\text { grade }\end{array}$} & \multicolumn{2}{|c|}{$\begin{array}{l}\text { Extra-high- } \\
\text { strength grade }\end{array}$} & \multicolumn{2}{|c|}{$\begin{array}{l}\text { High-strength } \\
\text { grade }\end{array}$} \\
\hline & $m m$ & $7 b / f t$ & $\mathrm{~kg} / \mathrm{m}$ & $\begin{array}{l}\text { Tons of } \\
2,000 \mathrm{lb}\end{array}$ & $\begin{array}{l}\text { Tons of } \\
1.000 \mathrm{~kg}\end{array}$ & $\begin{array}{l}\text { Tons of } \\
2,000 \text { ib }\end{array}$ & $\begin{array}{l}\text { Tons of } \\
1,000 \mathrm{~kg}\end{array}$ & $\begin{array}{l}\text { Tons of } \\
\stackrel{2}{2}, 000 \mathrm{lb}\end{array}$ & $\begin{array}{c}\text { Tons of } \\
1,000 \mathrm{~kg}\end{array}$ & $\begin{array}{l}\text { Tons of } \\
2,000 \mathrm{lb}\end{array}$ & $\begin{array}{l}\text { Tons of } \\
1,000 \mathrm{~kg}\end{array}$ \\
\hline & 12.7 & 0.55 & 0.82 & 15.3 & 13.9 & 12.6 & 11.4 & 17.0 & 15.4 & 14.0 & 12.7 \\
\hline $5 / 8$ & 15.9 & .86 & 1.28 & 22.3 & 20.2 & 19.2 & 17.4 & 24.8 & 22.4 & 21.3 & 19.3 \\
\hline $3 / 4$ & 19.0 & 1. 24 & 1.84 & 32.5 & 29.5 & 27.6 & 25. & 36.1 & 32.8 & 30.7 & 27.8 \\
\hline $7 / 8$ & 22.2 & 1. 69 & 2.51 & 44.4 & 40.3 & 37.6 & 34.1 & 49.3 & 44.8 & 41.8 & 37.9 \\
\hline 1 & 25.4 & 2. 20 & 3. 27 & 58.0 & 52.6 & 49. 2 & 44.6 & 64.4 & 58.4 & 54.7 & 49.5 \\
\hline $1 \frac{1}{8}$ & 28.6 & 2. 70 & 4. 02 & 70.7 & 64.1 & 60.0 & 54.4 & 78.5 & 71.2 & 55.7 & 60.4 \\
\hline $11 / 4$ & 31.7 & 3.23 & 4.81 & 84.6 & 76.7 & 71.8 & 65.1 & 94.0 & 85.2 & 79.8 & 72.3 \\
\hline $13 / 8$ & 34.9 & 4. 01 & 5.97 & 105.0 & 95.2 & 88.8 & 80.5 & 116. 7 & 105.8 & 98.7 & 89.4 \\
\hline 11 & 38.1 & 4.88 & 7. 26 & 127.5 & 115.7 & 108.4 & 98.3 & 141.7 & 128.5 & 120.4 & 109.2 \\
\hline 15 & 41.3 & 5. 63 & 8.38 & 146.0 & 132.4 & 124.0 & 112.5 & 162.2 & 147.1 & 137.8 & 125.0 \\
\hline $13 / 1$ & 44. & 6.5 & 9. & I71. 0 & 155. & 145.8 & 132.3 & 190.0 & 17 & 0 & 147 \\
\hline $17 \% 8$ & 47. & 7. 28 & 10. & 189.0 & 171.4 & 161 & 146.0 & 210.0 & 190 & 178.9 & 162. 2 \\
\hline & 50.8 & 8.40 & 12.50 & 218.0 & 197.8 & 185.0 & 167.8 & 242.2 & 219 & 205.5 & 186.4 \\
\hline $21 / 8$ & 54.0 & 9.35 & 13.91 & 240.0 & 217.7 & 204.0 & 185.1 & 266.7 & 241.9 & 226.7 & 205.7 \\
\hline $21 / 1$ & 57.1 & 10.36 & 15.42 & 266.0 & 241.3 & 233.0 & 211.4 & 295.5 & 268.1 & 258.9 & 234.9 \\
\hline $21 / 2$ & 63.5 & 13.10 & 19.49 & 335.0 & 303.9 & 285.0 & 258.5 & 372.2 & 337.7 & 316.7 & 287.2 \\
\hline
\end{tabular}




\begin{tabular}{|c|c|c|c|c|c|c|}
\hline & $\approx$ & 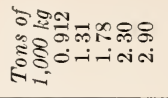 & 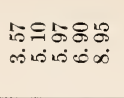 & 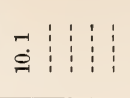 & & $\begin{array}{l:c}\vdots \\
\vdots \\
\vdots\end{array}$ \\
\hline 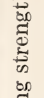 & $\Xi$ & 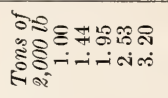 & 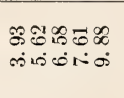 & 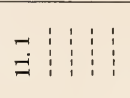 & & \\
\hline 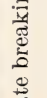 & $\bar{\Phi}$ & 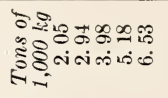 & 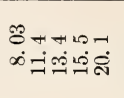 & 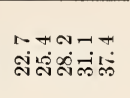 & 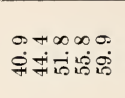 & 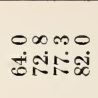 \\
\hline 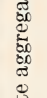 & $\frac{3}{2}$ & 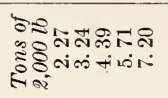 & 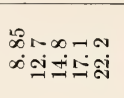 & 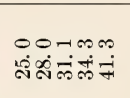 & 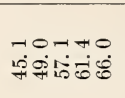 & 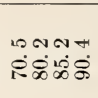 \\
\hline 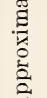 & 产 & 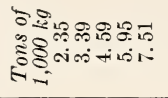 & 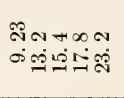 & 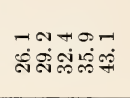 & 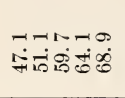 & 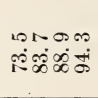 \\
\hline & 它 & 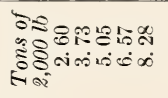 & 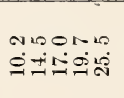 & 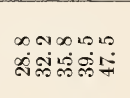 & 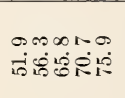 & 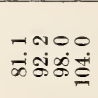 \\
\hline \multirow{6}{*}{ 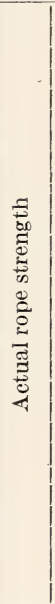 } & \multirow{2}{*}{ Е } & 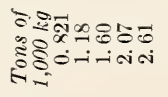 & 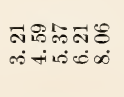 & $\begin{array}{lll}t & \\
o & & \end{array}$ & & \\
\hline & & 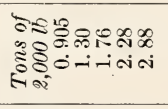 & 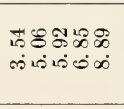 & $\stackrel{\circ}{9}$ & & \\
\hline & \multirow{2}{*}{ 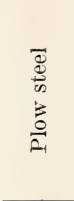 } & 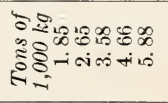 & 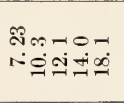 & 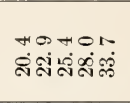 & 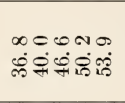 & $\begin{array}{l}0100 \infty \\
15808 \\
158\end{array}$ \\
\hline & & 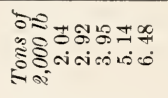 & 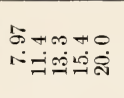 & 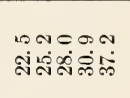 & 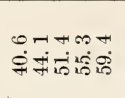 & 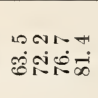 \\
\hline & \multirow{2}{*}{ 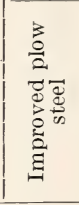 } & 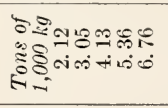 & 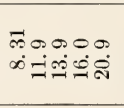 & 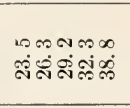 & 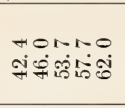 & 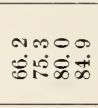 \\
\hline & & 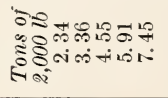 & 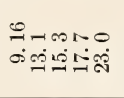 & 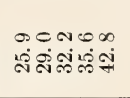 & 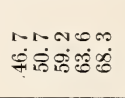 & 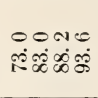 \\
\hline \multirow{2}{*}{\multicolumn{2}{|c|}{ 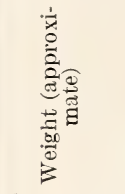 }} & ำำตำำ & 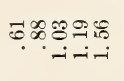 & 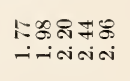 & 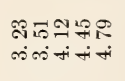 & 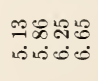 \\
\hline & & ㅇำ & 구요요 & 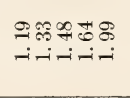 & 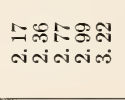 & 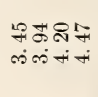 \\
\hline \multirow{2}{*}{\multicolumn{2}{|c|}{ 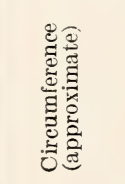 }} & 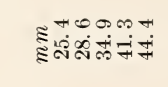 & 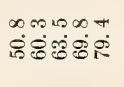 & 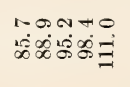 & 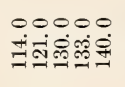 & 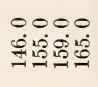 \\
\hline & & 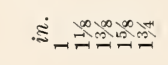 & 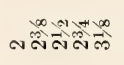 & $\begin{array}{l}\infty \\
\infty \\
\infty \\
\infty\end{array}$ & 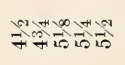 & की \\
\hline \multirow{2}{*}{\multicolumn{2}{|c|}{ 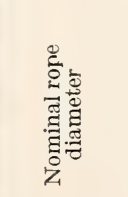 }} & 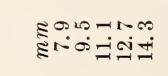 & 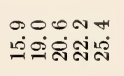 & 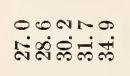 & 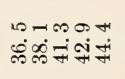 & 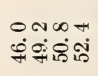 \\
\hline & & : & 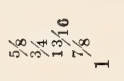 & $\stackrel{0}{\infty}=0$ & 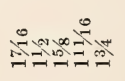 & 正 \\
\hline
\end{tabular}




\begin{tabular}{|c|c|c|c|c|}
\hline \multirow{4}{*}{ 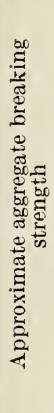 } & \multirow{2}{*}{ 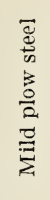 } & 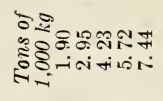 & 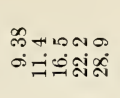 & 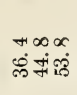 \\
\hline & & 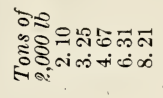 & 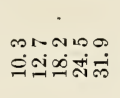 & 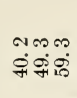 \\
\hline & \multirow{2}{*}{$\begin{array}{l}\vec{\Phi} \\
\text { in } \\
\vdots \\
\vdots \\
0 \\
0\end{array}$} & 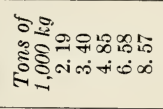 & 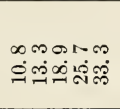 & 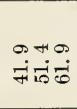 \\
\hline & & 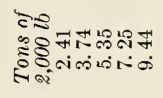 & 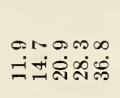 & 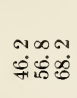 \\
\hline \multirow{4}{*}{ 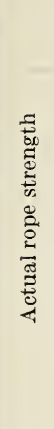 } & \multirow{2}{*}{ 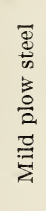 } & 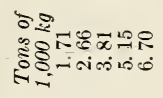 & 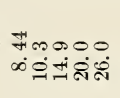 & 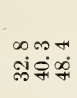 \\
\hline & & 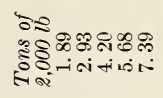 & 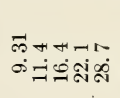 & 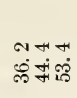 \\
\hline & \multirow{2}{*}{ 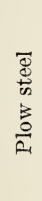 } & 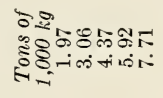 & 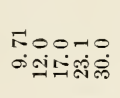 & 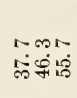 \\
\hline & & 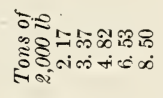 & 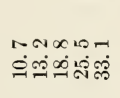 & नंम्ये \\
\hline \multirow{2}{*}{\multicolumn{2}{|c|}{ 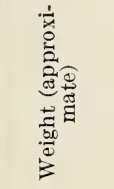 }} & 통 & 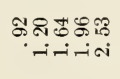 & 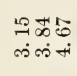 \\
\hline & & 도융ำ & 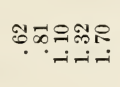 & 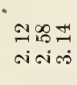 \\
\hline \multirow{2}{*}{\multicolumn{2}{|c|}{ 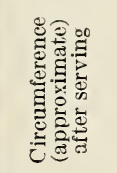 }} & 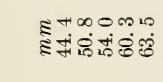 & $\begin{array}{l}\infty+\infty \pi 0 \\
\dot{B} \dot{\infty} \infty \dot{\Xi}\end{array}$ & 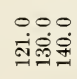 \\
\hline & & 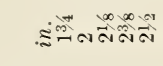 & 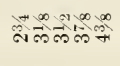 & 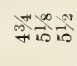 \\
\hline \multirow{4}{*}{ 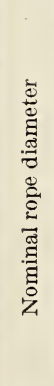 } & \multirow{2}{*}{ 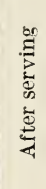 } & 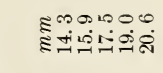 & 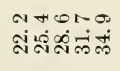 & 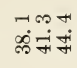 \\
\hline & & 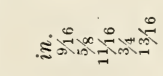 & $\stackrel{\infty}{\infty} \underset{-\infty}{\infty}$ & 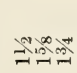 \\
\hline & \multirow{2}{*}{ 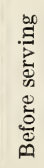 } & 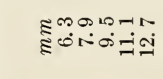 & 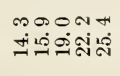 & 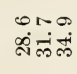 \\
\hline & & . & mism, & 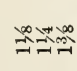 \\
\hline
\end{tabular}

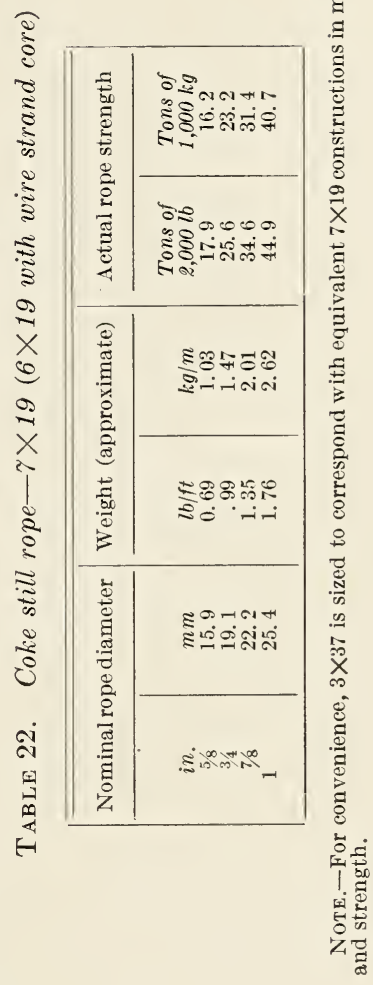

$823512-49-5$ 
TaBle 23. Torpedo lines $-5 \times 5$

\begin{tabular}{|c|c|c|c|c|c|c|c|c|c|c|c|}
\hline \multirow{2}{*}{\multicolumn{2}{|c|}{$\begin{array}{c}\text { Nominal rope } \\
\text { diameter }\end{array}$}} & \multirow{2}{*}{\multicolumn{2}{|c|}{$\begin{array}{l}\text { Weight (ap- } \\
\text { proximate) }\end{array}$}} & \multicolumn{4}{|c|}{ Actual rope strength } & \multicolumn{4}{|c|}{$\begin{array}{c}\text { Approximate aggregate breaking } \\
\text { strength }\end{array}$} \\
\hline & & & & \multicolumn{2}{|c|}{$\begin{array}{c}\text { Improved plow } \\
\text { steel }\end{array}$} & \multicolumn{2}{|c|}{ Plow steel } & \multicolumn{2}{|c|}{$\begin{array}{c}\text { Improved plow } \\
\text { steel }\end{array}$} & \multicolumn{2}{|c|}{ Plow steel } \\
\hline in. & $m m$ & $\begin{array}{c}l b / 100 \\
f t\end{array}$ & $\begin{array}{c}\mathrm{kg} / 100 \\
\mathrm{~m}\end{array}$ & $\begin{array}{l}\text { Tons of } \\
2,000 \mathrm{lb}\end{array}$ & $\begin{array}{c}\text { Tons of } \\
1,000 \mathrm{~kg}\end{array}$ & $\begin{array}{l}\text { Tons of } \\
2,000 \text { lb }\end{array}$ & $\begin{array}{c}\text { Tons of } \\
1,000 \mathrm{~kg}\end{array}$ & $\begin{array}{l}\text { Tons of } \\
2,000 \mathrm{lb}\end{array}$ & $\begin{array}{l}\text { Tons of } \\
1,000 \mathrm{~kg}\end{array}$ & $\begin{array}{l}\text { Tons of } \\
2,000 \text { lb }\end{array}$ & $\begin{array}{c}\text { Tons of } \\
1,000 \mathrm{~kg}\end{array}$ \\
\hline 1,8 & 3.2 & 2.2 & 3.3 & 0.625 & 0.567 & 0.55 & $\begin{array}{c}1,499 \\
0.49\end{array}$ & 0.725 & 0.659 & 0.64 & 0.580 \\
\hline 964 & 3.6 & 2.7 & $4 .($ & .800 & .726 & .70 & .635 & .930 & .844 & .815 & .738 \\
\hline $5 / 32$ & 4. & 3.3 & 4. & 5 & .8 & .85 & .7 & 1. 135 & 1.030 & .99 & .896 \\
\hline $3 / 16$ & 4. & 5.0 & 7.4 & 1.450 & 1.310 & 1. 25 & 1.130 & 1.685 & 1.520 & 1.455 & 1. 310 \\
\hline $1 / 4$ & 6.3 & 8.3 & 12.3 & 2. 400 & 2. 180 & 2. 10 & 1.900 & 2. 790 & 2. 530 & 2.44 & 2.210 \\
\hline
\end{tabular}

TABLE 24. Galvanized steel strand-7-wire strand

\begin{tabular}{|c|c|c|c|c|c|c|c|c|c|c|c|}
\hline \multirow{2}{*}{\multicolumn{2}{|c|}{$\begin{array}{l}\text { Nominal strand } \\
\text { diameter }\end{array}$}} & \multirow{2}{*}{\multicolumn{2}{|c|}{$\begin{array}{l}\text { Weight (ap- } \\
\text { proximate) }\end{array}$}} & \multicolumn{4}{|c|}{ Actual strand strength } & \multicolumn{4}{|c|}{$\begin{array}{c}\text { Approximate aggregate breaking } \\
\text { strength }\end{array}$} \\
\hline & & & & \multicolumn{2}{|c|}{ Common grade } & \multicolumn{2}{|c|}{$\begin{array}{l}\text { Siemens-Martin } \\
\text { grade }\end{array}$} & \multicolumn{2}{|c|}{ Common grade } & \multicolumn{2}{|c|}{$\begin{array}{l}\text { Siemens-Martin } \\
\text { grade }\end{array}$} \\
\hline $\begin{array}{l}\text { in. } \\
1 / 8 \\
5 / 32 \\
3 / 16 \\
7 / 32 \\
1 / 4\end{array}$ & $\begin{array}{l}m m \\
3.2 \\
4.0 \\
4.8 \\
5.5 \\
6.3\end{array}$ & $\begin{array}{c}l b / 1,000 \\
f t \\
31.8 \\
51.3 \\
72.9 \\
98.3 \\
121.0\end{array}$ & $\begin{array}{c}\mathrm{kg} / 100 \mathrm{~m} \\
4.73 \\
7.63 \\
10.8 \\
14.6 \\
18.0\end{array}$ & $\begin{array}{r}\text { Tons of } \\
2,000 \mathrm{lb} \\
0.270 \\
.435 \\
.575 \\
.770 \\
.950\end{array}$ & $\begin{array}{c}\text { Tons of } \\
1,000 \mathrm{~kg} \\
0.245 \\
.395 \\
.522 \\
.698 \\
.862\end{array}$ & $\begin{array}{r}\text { Tons of } \\
2,000 \mathrm{lb} \\
0.455 \\
.735 \\
.950 \\
1.280 \\
1.575\end{array}$ & $\begin{array}{c}\text { Tons of } \\
1,000 \mathrm{~kg} \\
0.413 \\
.667 \\
.862 \\
1.160 \\
1.430\end{array}$ & $\begin{array}{c}\text { Tons of } \\
2,000 \mathrm{lb} \\
0.281 \\
.453 \\
.600 \\
.800 \\
.990\end{array}$ & $\begin{array}{c}\text { Tons of } \\
1,000 \mathrm{~kg} \\
.255 \\
.411 \\
.544 \\
.727 \\
.898\end{array}$ & $\begin{array}{c}\text { Tons of } \\
2,000 \mathrm{lb} \\
0.474 \\
.765 \\
.990 \\
1.335 \\
1.640\end{array}$ & $\begin{array}{c}\text { Tons of } \\
1,000 \mathrm{~kg} \\
0.430 \\
.695 \\
.898 \\
1.210 \\
1.490\end{array}$ \\
\hline $\begin{array}{l}9 / 32 \\
5 / 16 \\
3 / 8 \\
7 / 16 \\
1 / 2\end{array}$ & $\begin{array}{r}7.1 \\
7.9 \\
9.5 \\
11.1 \\
12.7\end{array}$ & $\begin{array}{l}164.0 \\
205.0 \\
273.0 \\
399.0 \\
517.0\end{array}$ & $\begin{array}{l}24.4 \\
30.5 \\
40.6 \\
59.4 \\
76.9\end{array}$ & $\begin{array}{l}1.285 \\
1.600 \\
2.125 \\
2.850 \\
3.700\end{array}$ & $\begin{array}{l}1.160 \\
1.450 \\
1.930 \\
2.580 \\
3.360\end{array}$ & $\begin{array}{l}2.125 \\
2.675 \\
3.475 \\
4.675 \\
6.050\end{array}$ & $\begin{array}{l}1.930 \\
2.430 \\
3.150 \\
4.240 \\
5.490\end{array}$ & $\begin{array}{l}1.340 \\
1.665 \\
2.215 \\
2.970 \\
3.855\end{array}$ & $\begin{array}{l}1.210 \\
1.510 \\
2.010 \\
2.690 \\
3.500\end{array}$ & $\begin{array}{l}2.215 \\
2.785 \\
3.620 \\
4.870 \\
6.300\end{array}$ & $\begin{array}{l}2.010 \\
2.530 \\
3.280 \\
4.420 \\
5.720\end{array}$ \\
\hline \multirow[t]{2}{*}{$\begin{array}{l}9 / 16 \\
58\end{array}$} & \multirow[t]{2}{*}{$\begin{array}{l}14.3 \\
15.9\end{array}$} & \multirow[t]{2}{*}{$\begin{array}{l}671.0 \\
813.0\end{array}$} & \multirow[t]{2}{*}{$\begin{array}{r}99.8 \\
121.0\end{array}$} & $\begin{array}{l}4.800 \\
5.800\end{array}$ & $\begin{array}{l}4.350 \\
5.260\end{array}$ & $\begin{array}{l}7.850 \\
9.550\end{array}$ & $\begin{array}{l}7.120 \\
8.660\end{array}$ & $\begin{array}{l}5.000 \\
6.050\end{array}$ & $\begin{array}{l}4.530 \\
5.480\end{array}$ & $\begin{array}{l}8.150 \\
9.950\end{array}$ & $\begin{array}{l}7.420 \\
9.020\end{array}$ \\
\hline & & & & \multicolumn{2}{|c|}{$\begin{array}{l}\text { High-strength } \\
\text { grade }\end{array}$} & \multicolumn{2}{|c|}{$\begin{array}{l}\text { Extra-high- } \\
\text { strength grade }\end{array}$} & \multicolumn{2}{|c|}{$\begin{array}{l}\text { High-strength } \\
\text { grade }\end{array}$} & \multicolumn{2}{|c|}{$\begin{array}{l}\text { Extra-high- } \\
\text { strength grade }\end{array}$} \\
\hline $\begin{array}{l}1 / 8 \\
5 / 32 \\
3 / 16 \\
7 / 32 \\
1 / 4\end{array}$ & $\begin{array}{l}3.2 \\
4.0 \\
4.8 \\
5.5 \\
6.3\end{array}$ & $\begin{array}{r}31.8 \\
51.3 \\
72.9 \\
98.3 \\
121.0\end{array}$ & $\begin{array}{l}4.73 \\
7.63 \\
10.8 \\
14.6 \\
18.0\end{array}$ & $\begin{array}{r}\text { Tons of } \\
2,000 \mathrm{lb} \\
0.665 \\
1.070 \\
1.425 \\
1.925 \\
2.375\end{array}$ & $\begin{array}{c}\text { Tons of } \\
1,000 \mathrm{~kg} \\
0.603 \\
.971 \\
1.290 \\
1.750 \\
2.150\end{array}$ & $\begin{array}{c}\text { Tons of } \\
2,000 \mathrm{lb} \\
0.915 \\
1.470 \\
1.995 \\
2.700 \\
3.325\end{array}$ & $\begin{array}{c}\text { Tons of } \\
1,000 \mathrm{~kg} \\
0.830 \\
1.330 \\
1.810 \\
2.450 \\
3.020\end{array}$ & $\begin{array}{c}\text { Tons of } \\
2,000 \mathrm{lb} \\
0.690 \\
1.115 \\
1.485 \\
2.005 \\
2.475\end{array}$ & $\begin{array}{c}\text { Tons of } \\
1,000 \mathrm{~kg} \\
0.628 \\
1.010 \\
1.340 \\
1.820 \\
2.240\end{array}$ & $\begin{array}{c}\text { Tons of } \\
2,000 \mathrm{lb} \\
0.955 \\
1.530 \\
2.080 \\
2.810 \\
3.465\end{array}$ & $\begin{array}{c}\text { Tons of } \\
1,000 \mathrm{~kg} \\
0.864 \\
1.380 \\
1.880 \\
2.550 \\
3.140\end{array}$ \\
\hline $\begin{array}{l}9 / 32 \\
51 / 6 \\
3 / 8 \\
7 / 16 \\
1 / 2\end{array}$ & $\begin{array}{r}7.1 \\
7.9 \\
9.5 \\
11.1 \\
12.7\end{array}$ & $\begin{array}{l}164.0 \\
205.0 \\
273.0 \\
399.0 \\
517.0\end{array}$ & $\begin{array}{l}24.4 \\
30.5 \\
40.6 \\
59.4 \\
76.9\end{array}$ & $\begin{array}{l}3.200 \\
4.000 \\
5.400 \\
7.250 \\
9.400\end{array}$ & $\begin{array}{l}2.900 \\
3.630 \\
4.900 \\
6.580 \\
8.530\end{array}$ & $\begin{array}{r}4.475 \\
5.600 \\
7.700 \\
10.400 \\
13.450\end{array}$ & $\begin{array}{r}4.060 \\
5.080 \\
6.980 \\
9.430 \\
12.200\end{array}$ & $\begin{array}{l}3.335 \\
4.165 \\
5.600 \\
7.550 \\
9.800\end{array}$ & $\begin{array}{l}3.020 \\
3.780 \\
5.100 \\
6.850 \\
8.880\end{array}$ & $\begin{array}{r}4.660 \\
5.850 \\
8.000 \\
10.850 \\
14.000\end{array}$ & $\begin{array}{r}4.230 \\
5.290 \\
7.270 \\
9.820 \\
12.700\end{array}$ \\
\hline $\begin{array}{l}9 / 16 \\
5 / 8\end{array}$ & $\begin{array}{l}14.3 \\
15.9\end{array}$ & $\begin{array}{l}671.0 \\
813.0\end{array}$ & $\begin{array}{r}99.8 \\
121.0\end{array}$ & $\begin{array}{l}12.250 \\
14.800\end{array}$ & $\begin{array}{l}11.100 \\
13.400\end{array}$ & $\begin{array}{l}17.500 \\
21.200\end{array}$ & $\begin{array}{l}15.900 \\
19.200\end{array}$ & $\begin{array}{l}12.750 \\
15.400\end{array}$ & $\begin{array}{l}11.600 \\
13.900\end{array}$ & $\begin{array}{l}18.200 \\
22.100\end{array}$ & $\begin{array}{l}16.600 \\
20.000\end{array}$ \\
\hline
\end{tabular}


TABLE 25. Galvanized steel strand-19-wire strand

\begin{tabular}{|c|c|c|c|c|c|c|c|c|c|c|c|}
\hline \multirow{2}{*}{\multicolumn{2}{|c|}{$\begin{array}{l}\text { Nominal strand } \\
\text { diameter }\end{array}$}} & \multirow{2}{*}{\multicolumn{2}{|c|}{$\begin{array}{l}\text { Weight (ap- } \\
\text { proximate) }\end{array}$}} & \multicolumn{4}{|c|}{ Actual strand strength } & \multicolumn{4}{|c|}{$\begin{array}{l}\text { Approximate aggregate breaking } \\
\text { strength }\end{array}$} \\
\hline & & & & \multicolumn{2}{|c|}{ Common grade } & \multicolumn{2}{|c|}{$\underset{\text { grade }}{\text { Siemens-Martin }}$} & \multicolumn{2}{|c|}{ Common grade } & \multicolumn{2}{|c|}{$\begin{array}{l}\text { Siemens-Martin } \\
\text { grade }\end{array}$} \\
\hline $\begin{array}{l}\text { in } \\
1 / 2 \\
91 / 6 \\
5 / 8 \\
3 / 4 \\
7 / 8\end{array}$ & $\begin{array}{l}m m \\
12.7 \\
14.3 \\
15.9 \\
19.0 \\
22.2\end{array}$ & $\begin{array}{c}l b / 1,000 \\
f t \\
504.0 \\
637.0 \\
796.0 \\
1155.0 \\
1581.0\end{array}$ & $\begin{array}{c}\mathrm{kg} / 100 \mathrm{~m} \\
75.0 \\
94.8 \\
118.0 \\
172.0 \\
235.0\end{array}$ & $\begin{array}{c}\text { Tons of } \\
2,000 \mathrm{lb} \\
3.81 \\
4.82 \\
5.50 \\
8.00 \\
10.95\end{array}$ & $\begin{array}{c}\text { Tons of } \\
1,000 \mathrm{~kg} \\
3.460 \\
4.370 \\
4.990 \\
7.260 \\
9.930\end{array}$ & \begin{tabular}{|c|} 
Tons of \\
$2,000 \mathrm{lb}$ \\
6.35 \\
8.05 \\
9.05 \\
13.10 \\
17.95
\end{tabular} & $\begin{array}{r}\text { Tons of } \\
1,000 \mathrm{~kg} \\
5.760 \\
7.300 \\
8.210 \\
11.900 \\
16.300\end{array}$ & $\begin{array}{c}\text { Tcns of } \\
2,000 \mathrm{lb} \\
4.05 \\
5.10 \\
5.85 \\
8.50 \\
11.65\end{array}$ & $\begin{array}{c}\text { Tons of } \\
1,000 \mathrm{~kg} \\
3.680 \\
4.650 \\
5.310 \\
7.720 \\
10.600\end{array}$ & $\begin{array}{c}\text { Tons of } \\
2,000 \mathrm{lb} \\
6.75 \\
8.55 \\
9.60 \\
13.95 \\
19.10\end{array}$ & $\begin{array}{r}\text { Tons of } \\
1,000 \mathrm{~kg} \\
6.130 \\
7.760 \\
8.730 \\
12.600 \\
17.300\end{array}$ \\
\hline \multirow{3}{*}{1} & \multirow[t]{3}{*}{25.4} & \multirow[t]{3}{*}{2073.0} & \multirow[t]{3}{*}{308.0} & 14.35 & 13.000 & 23.50 & 21.300 & 15.25 & 13.800 & 25.00 & 22.600 \\
\hline & & & & \multicolumn{2}{|c|}{$\begin{array}{l}\text { High-strength } \\
\text { grade }\end{array}$} & \multicolumn{2}{|c|}{$\begin{array}{c}\text { Extra-high- } \\
\text { strength grade }\end{array}$} & \multicolumn{2}{|c|}{$\begin{array}{l}\text { High-strength } \\
\text { grade }\end{array}$} & \multicolumn{2}{|c|}{$\begin{array}{l}\text { Extra-high- } \\
\text { strength grade }\end{array}$} \\
\hline & & & & $\begin{array}{l}\text { Tons of } \\
2,000 \mathrm{lb}\end{array}$ & $\begin{array}{c}\text { Tons of } \\
1,000 \mathrm{~kg}\end{array}$ & $\begin{array}{l}\text { T ons of } \\
2,000 \text { lb }\end{array}$ & $\left|\begin{array}{c}\text { Tons of } \\
1,000 \mathrm{~kg}\end{array}\right|$ & $\begin{array}{l}\text { Tons of } \\
2,000 \mathrm{lb}\end{array}$ & $\left|\begin{array}{c}\text { Tons of } \\
1,000 \mathrm{~kg}\end{array}\right|$ & $\begin{array}{l}\text { Tons of } \\
2,000 \mathrm{lb}\end{array}$ & $\begin{array}{c}\text { Tons of } \\
1,000 \mathrm{~kg}\end{array}$ \\
\hline$\frac{1 / 2}{9 / 6}$ & $\begin{array}{l}12.7 \\
14.3\end{array}$ & $\begin{array}{l}504.0 \\
637.0\end{array}$ & $\begin{array}{l}75.0 \\
94.8\end{array}$ & $\begin{array}{r}9.550 \\
12.050\end{array}$ & $\begin{array}{r}8.660^{\circ} \\
10.900\end{array}$ & $\begin{array}{l}13.350 \\
16.850\end{array}$ & 12. 100 & 10.150 & $\begin{array}{r}9.210^{\circ} \\
11.600\end{array}$ & 14. 200 & 12. 900 \\
\hline $5 / 8$ & $\begin{array}{l}14.3 \\
15.9\end{array}$ & $\begin{array}{l}037.0 \\
796.0\end{array}$ & $\begin{array}{r}94.8 \\
118.0\end{array}$ & $\begin{array}{l}12.050 \\
14.050\end{array}$ & 12.7 & $\begin{array}{l}10.8 \\
20.1\end{array}$ & $\begin{array}{l}15 . \\
18 .\end{array}$ & 14. & $\begin{array}{l}11.000 \\
13.500\end{array}$ & 21. & $\begin{array}{l}16.300 \\
19.400\end{array}$ \\
\hline $3 / 4$ & 19.0 & 1155.0 & 172.0 & 20.400 & 18. 500 & 29.150 & 26.400 & 21. 700 & & 31.000 & 28.100 \\
\hline $7 / 8$ & 22.2 & 1581.0 & 235.0 & 27.900 & 25.300 & 39.850 & 36.100 & 29.700 & 26.900 & 42.400 & 38.400 \\
\hline 1 & 25.4 & 2073.0 & 308.0 & 36.600 & 33.200 & 52.250 & 47.400 & 38.950 & 35.300 & 55.500 & 50.400 \\
\hline
\end{tabular}

TABLE 26. Galvanized steel strand, utilities grade-3-and 7 -wire strand

\begin{tabular}{|c|c|c|c|c|c|c|c|c|}
\hline \multirow{2}{*}{ Wire strand } & \multicolumn{2}{|c|}{$\begin{array}{l}\text { Nominal strand } \\
\text { diameter }\end{array}$} & \multicolumn{2}{|c|}{$\begin{array}{l}\text { Weight (approxi- } \\
\text { mate) }\end{array}$} & \multicolumn{2}{|c|}{$\begin{array}{l}\text { Actual strand } \\
\text { strength } 1\end{array}$} & \multicolumn{2}{|c|}{$\begin{array}{l}\text { Approximate ag- } \\
\text { gregate break- } \\
\text { ing strength }\end{array}$} \\
\hline & $\begin{array}{l}\text { in. } \\
1 / 4 \\
1 / 4 \\
5 / 16 \\
3 / 8\end{array}$ & $\begin{array}{l}m m \\
6.3 \\
6.3 \\
7.9 \\
9.5\end{array}$ & $\begin{array}{c}l b / 1,000 \mathrm{ft} \\
116.7 \\
116.7 \\
170.6 \\
220.3\end{array}$ & $\begin{array}{c}\mathrm{kg} / 100 \mathrm{~m} \\
17.4 \\
17.4 \\
25.4 \\
32.8\end{array}$ & $\begin{array}{l}\text { Tons of } \\
2,000 \text { lb } \\
\text { a } 1.575 \\
\text { b } 2.250 \\
\text { b } 3.250 \\
\text { b } 4.250\end{array}$ & $\begin{array}{c}\text { Tons of } \\
1,000 \mathrm{~kg} \\
1.430 \\
2.040 \\
2.950 \\
3.850\end{array}$ & $\begin{array}{c}\text { Tons of } \\
2,000 \mathrm{lb} \\
1.640 \\
2.345 \\
3.385 \\
4.425\end{array}$ & $\begin{array}{c}\text { Tons of } \\
1,000 \mathrm{~kg} \\
1.490 \\
2.120 \\
3.070 \\
4.010\end{array}$ \\
\hline & $\begin{array}{l}3 / 16 \\
9 / 32 \\
5 / 16 \\
3 / 8 \\
7 / 16 \\
1 / 2\end{array}$ & $\begin{array}{r}4.8 \\
7.1 \\
7.9 \\
9.5 \\
11.1 \\
12.7\end{array}$ & $\begin{array}{r}80.3 \\
164.0 \\
225.0 \\
273.0 \\
399.0 \\
517.0\end{array}$ & $\begin{array}{l}11.9 \\
24.4 \\
33.5 \\
40.6 \\
59.4 \\
76.9\end{array}$ & $\begin{array}{r}\text { c } 1.200 \\
\text { e } 2.300 \\
\text { e } 3.000 \\
\text { d } 5.750 \\
\text { d } 9.000 \\
\text { d } 12.500\end{array}$ & $\begin{array}{r}1.090 \\
2.090 \\
2.720 \\
5.220 \\
8.160 \\
11.300\end{array}$ & $\begin{array}{r}1.250 \\
2.395 \\
3.125 \\
6.000 \\
9.350 \\
13.000\end{array}$ & $\begin{array}{r}1.130 \\
2.180 \\
2.830 \\
5.440 \\
8.500 \\
11.800\end{array}$ \\
\hline
\end{tabular}

1 Minimum elongation requirements:

a 8 percent in 24 inches $(61 \mathrm{~cm})$.

b 5 percent in 24 inches $(61 \mathrm{~cm})$.

c 10 percent in 24 inches $(61 \mathrm{~cm})$.

d 4 percent in 24 inches $(61 \mathrm{~cm})$. 
TABLE 27. Galvanized bridge strand

\begin{tabular}{|c|c|c|c|c|c|c|c|}
\hline \multicolumn{2}{|c|}{$\begin{array}{l}\text { Nominal strand } \\
\text { diameter }\end{array}$} & \multicolumn{2}{|c|}{ Weight (approximate) ${ }^{1}$} & \multicolumn{2}{|c|}{ Actual strand strength } & \multicolumn{2}{|c|}{$\begin{array}{c}\text { Approximate metallic } \\
\text { area } 1\end{array}$} \\
\hline $\begin{array}{l}i n . \\
1 / 2 \\
9 / 16 \\
5 / 8 \\
3 / 4 \\
7 / 8\end{array}$ & $\begin{array}{l}m m \\
12.7 \\
14.3 \\
15.9 \\
19.0 \\
22.2\end{array}$ & $\begin{array}{r}l b / f t \\
0.52 \\
.66 \\
.81 \\
1.16 \\
1.56\end{array}$ & $\begin{array}{l}\mathrm{kg} / \mathrm{m} \\
0.77 \\
.98 \\
1.20 \\
1.73 \\
2.32\end{array}$ & $\begin{array}{c}\text { Tons of } \\
2,000 \mathrm{lb} \\
15.0 \\
19.0 \\
24.0 \\
34.0 \\
46.0\end{array}$ & $\begin{array}{c}\text { Tons of } \\
1,000 \mathrm{~kg} \\
13.6 \\
17.2 \\
21.8 \\
30.8 \\
41.7\end{array}$ & $\begin{array}{c}s q \text { in. } \\
0.149 \\
.189 \\
.233 \\
.333 \\
.450\end{array}$ & $\begin{array}{r}s q m m \\
96.1 \\
122.0 \\
150.0 \\
215.0 \\
290.0\end{array}$ \\
\hline $\begin{array}{l}1 \\
11 / 8 \\
11 / 4 \\
13 / 8 \\
11 / 2\end{array}$ & $\begin{array}{l}25.4 \\
28.6 \\
31.7 \\
34.9 \\
38.1\end{array}$ & $\begin{array}{l}2.07 \\
2.64 \\
3.26 \\
3.94 \\
4.69\end{array}$ & $\begin{array}{l}3.08 \\
3.93 \\
4.85 \\
5.86 \\
6.98\end{array}$ & $\begin{array}{r}61.0 \\
78.0 \\
96.0 \\
116.0 \\
138.0\end{array}$ & $\begin{array}{r}55.3 \\
70.8 \\
87.1 \\
105.0 \\
125.0\end{array}$ & $\begin{array}{r}.596 \\
.760 \\
.940 \\
1.135 \\
1.350\end{array}$ & $\begin{array}{l}384.0 \\
490.0 \\
606.0 \\
732.0 \\
871.0\end{array}$ \\
\hline $\begin{array}{l}15 / 8 \\
13 / 4 \\
17 / 8 \\
2 \\
21 / 8\end{array}$ & $\begin{array}{l}41.3 \\
44.4 \\
47.6 \\
50.8 \\
54.0\end{array}$ & $\begin{array}{l}5.50 \\
6.38 \\
7.32 \\
8.34 \\
9.42\end{array}$ & $\begin{array}{r}8.18 \\
9.49 \\
10.89 \\
12.41 \\
14.02\end{array}$ & $\begin{array}{l}162.0 \\
188.0 \\
216.0 \\
245.0 \\
277.0\end{array}$ & $\begin{array}{l}147.0 \\
170.0 \\
196.0 \\
222.0 \\
251.0\end{array}$ & $\begin{array}{l}1.59 \\
1.84 \\
2.11 \\
2.40 \\
2.71\end{array}$ & $\begin{array}{l}1,026.0 \\
1,187.0 \\
1,361.0 \\
1,548.0 \\
1,748.0\end{array}$ \\
\hline $21 / 4$ & 57.1 & 10.55 & 15. 70 & 310.0 & 281.0 & 3.04 & $1,961.0$ \\
\hline
\end{tabular}

1 Weights and areas are only approximate. For exact values, refer to individual rope manufacturers.

TABLE 28. Galvanized bridge rope

\begin{tabular}{|c|c|c|c|c|c|c|c|}
\hline \multicolumn{2}{|c|}{ Nominal rope diameter } & \multicolumn{2}{|c|}{ Weight (approximate) } & \multicolumn{2}{|c|}{ Actual rope strength } & \multicolumn{2}{|c|}{$\begin{array}{c}\text { Approximate metallic } \\
\text { area }\end{array}$} \\
\hline $\begin{array}{l}\text { in. } \\
3 / 4 \\
7 / 8 \\
1^{11 / 8} \\
11 / 4\end{array}$ & $\begin{array}{l}m m \\
19.0 \\
22.2 \\
25.4 \\
28.6 \\
31.7\end{array}$ & $\begin{array}{l}l b / f t \\
0.95 \\
1.28 \\
1.67 \\
2.11 \\
2.64\end{array}$ & $\begin{array}{l}\mathrm{kg} / \mathrm{m} \\
1.41 \\
1.90 \\
2.48 \\
3.14 \\
3.93\end{array}$ & $\begin{array}{c}\text { Tons of } \\
2,000 \mathrm{lb} \\
26.0 \\
35.0 \\
45.7 \\
57.8 \\
72.2\end{array}$ & $\begin{array}{c}\text { Tons of } \\
1,000 \mathrm{~kg} \\
23.6 \\
31.7 \\
41.4 \\
52.4 \\
65.5\end{array}$ & $\begin{array}{l}s q i n . \\
0.268 \\
.361 \\
.471 \\
.596 \\
.745\end{array}$ & $\begin{array}{c}s q m m \\
173 \\
233 \\
304 \\
384 \\
481\end{array}$ \\
\hline $\begin{array}{l}13 / 8 \\
11 / 2 \\
15 / 8 \\
13 / 4 \\
17 / 8\end{array}$ & $\begin{array}{l}34.9 \\
38.1 \\
41.3 \\
44.4 \\
47.6\end{array}$ & $\begin{array}{l}\text { 3. } 21 \\
3.82 \\
4.51 \\
5.24 \\
6.03\end{array}$ & $\begin{array}{l}4.78 \\
5.68 \\
6.71 \\
7.80 \\
8.97\end{array}$ & $\begin{array}{r}87.8 \\
104.0 \\
123.0 \\
143.0 \\
164.0\end{array}$ & $\begin{array}{r}79.6 \\
94.3 \\
111.0 \\
130.0 \\
149.0\end{array}$ & $\begin{array}{l}.906 \\
1.076 \\
1.27 \\
1.47 \\
1.69\end{array}$ & $\begin{array}{r}584 \\
694 \\
819 \\
948 \\
1,090\end{array}$ \\
\hline $\begin{array}{l}2 \\
21 / 8 \\
21 / 4 \\
23 / 8 \\
21 / 2\end{array}$ & $\begin{array}{l}50.8 \\
54.0 \\
57.1 \\
60.3 \\
63.5\end{array}$ & $\begin{array}{r}6.85 \\
7.73 \\
8.66 \\
9.61 \\
10.60\end{array}$ & $\begin{array}{l}10.19 \\
11.50 \\
12.89 \\
14.30 \\
15.77\end{array}$ & $\begin{array}{l}186.0 \\
210.0 \\
235.0 \\
261.0 \\
288.0\end{array}$ & $\begin{array}{l}169.0 \\
190.0 \\
213.0 \\
237.0 \\
261.0\end{array}$ & $\begin{array}{l}1.92 \\
2.17 \\
2.42 \\
2.69 \\
2.97\end{array}$ & $\begin{array}{l}1,239 \\
1,400 \\
1,561 \\
1,735 \\
1,916\end{array}$ \\
\hline $\begin{array}{l}25 / 8 \\
23 / 4 \\
27 / 8 \\
3\end{array}$ & $\begin{array}{l}66.7 \\
69.8 \\
73.0 \\
76.2\end{array}$ & $\begin{array}{l}11.62 \\
12.74 \\
13.90 \\
15.11\end{array}$ & $\begin{array}{l}17.29 \\
18.96 \\
20.68 \\
22.49\end{array}$ & $\begin{array}{l}317.0 \\
347.0 \\
379.0 \\
412.0\end{array}$ & $\begin{array}{l}287.0 \\
315.0 \\
344.0 \\
374.0\end{array}$ & $\begin{array}{l}3.27 \\
3.58 \\
3.91 \\
4.25\end{array}$ & $\begin{array}{l}2,110 \\
2,310 \\
2,522 \\
2,742\end{array}$ \\
\hline
\end{tabular}


TABLE 29. $6 \times 7$ galvanized iron guy rope

\begin{tabular}{|c|c|c|c|c|c|c|c|c|c|}
\hline \multicolumn{2}{|c|}{$\begin{array}{l}\text { Nominal rope } \\
\text { diameter }\end{array}$} & \multicolumn{2}{|c|}{$\begin{array}{l}\text { Circumference } \\
\text { (approximate) }\end{array}$} & \multicolumn{2}{|c|}{$\begin{array}{l}\text { Weight (approxi- } \\
\text { mate) }\end{array}$} & \multicolumn{2}{|c|}{$\begin{array}{l}\text { Actual rope } \\
\text { strength }\end{array}$} & \multicolumn{2}{|c|}{$\begin{array}{l}\text { Approximate aggre- } \\
\text { gate breaking } \\
\text { strength }\end{array}$} \\
\hline $\begin{array}{l}\text { in. } \\
1 / 4 \\
5 / 16 \\
3 / 8 \\
7 / 16 \\
1 / 2\end{array}$ & $\begin{array}{r}m m \\
6.3 \\
7.9 \\
9.5 \\
11.1 \\
12.7\end{array}$ & $\begin{array}{l}\text { in. } \\
3 / 4 \\
1 \\
11 / 8 \\
138 \\
15 \% 8\end{array}$ & $\begin{array}{l}m m . \\
19.0 \\
25.4 \\
28.6 \\
34.9 \\
41.3\end{array}$ & $\begin{array}{l}l b / f t \\
0.094 \\
.15 \\
.21 \\
.29 \\
.38\end{array}$ & $\begin{array}{r}\mathrm{kg} / \mathrm{m} \\
0.14 \\
.22 \\
.31 \\
.43 \\
.56\end{array}$ & $\begin{array}{c}\text { Tons of } \\
2,000 \text { lb } \\
0.918 \\
1.42 \\
2.04 \\
2.76 \\
3.58\end{array}$ & $\begin{array}{c}\text { Tons of } \\
1,000 \mathrm{~kg} \\
0.833 \\
1.29 \\
1.85 \\
2.50 \\
3.25\end{array}$ & $\begin{array}{l}\text { Tons of } \\
2,000 \mathrm{lb} \\
1.07 \\
1.65 \\
2.37 \\
3.21 \\
4.16\end{array}$ & $\begin{array}{c}\text { Tons of } \\
1,000 \mathrm{~kg} \\
0.969 \\
1.50 \\
2.15 \\
2.91 \\
3.78\end{array}$ \\
\hline $\begin{array}{l}9 / 16 \\
558 \\
3 / 4 \\
13 / 16 \\
7 / 8\end{array}$ & $\begin{array}{l}14.3 \\
15.9 \\
19.0 \\
20.6 \\
22.2\end{array}$ & $\begin{array}{l}13 / 4 \\
2 \\
2^{3} / 8 \\
21 / 2 \\
2^{3} / 4\end{array}$ & $\begin{array}{l}44.4 \\
50.8 \\
60.3 \\
63.5 \\
69.8\end{array}$ & $\begin{array}{r}.48 \\
.59 \\
.84 \\
.99 \\
1.15\end{array}$ & $\begin{array}{r}.71 \\
.88 \\
1.25 \\
1.47 \\
1.71\end{array}$ & $\begin{array}{r}4.51 \\
5.54 \\
7.50 \\
9.23 \\
10.70\end{array}$ & $\begin{array}{l}4.09 \\
5.02 \\
7.17 \\
8.37 \\
9.71\end{array}$ & $\begin{array}{c}5.24 \\
6.44 \\
9.19 \\
10.7 \\
12.4\end{array}$ & $\begin{array}{r}4.75 \\
5.84 \\
8.34 \\
9.73 \\
11.3\end{array}$ \\
\hline $\begin{array}{l}1 \\
11 / 16 \\
11 / 8 \\
13 / 16 \\
11 / 4\end{array}$ & $\begin{array}{l}25.4 \\
27.0 \\
28.6 \\
30.2 \\
31.7\end{array}$ & $\begin{array}{l}31 / 8 \\
33,8 \\
31 / 2 \\
33 / 4 \\
37 / 8\end{array}$ & $\begin{array}{l}79.4 \\
85.7 \\
88.9 \\
95.2 \\
98.4\end{array}$ & $\begin{array}{l}1.50 \\
1.70 \\
1.90 \\
2.12 \\
2.34\end{array}$ & $\begin{array}{l}2.23 \\
2.53 \\
2.83 \\
3.15 \\
3.48\end{array}$ & $\begin{array}{l}13.8 \\
15.5 \\
17.3 \\
19.2 \\
21.2\end{array}$ & $\begin{array}{l}12.5 \\
14.1 \\
15.7 \\
17.4 \\
19.2\end{array}$ & $\begin{array}{l}16.0 \\
18.0 \\
20.1 \\
22.3 \\
24.6\end{array}$ & $\begin{array}{l}14.5 \\
16.4 \\
18.2 \\
20.2 \\
22.3\end{array}$ \\
\hline
\end{tabular}

TABLE 30. Galvanized spring lay rope

\begin{tabular}{|c|c|c|c|c|c|c|c|c|c|}
\hline \multicolumn{2}{|c|}{$\begin{array}{l}\text { Nominal rope } \\
\text { diameter }\end{array}$} & \multicolumn{2}{|c|}{$\begin{array}{l}\text { Circumference } \\
\text { (approximate) }\end{array}$} & \multicolumn{2}{|c|}{$\begin{array}{l}\text { Weight (approxi- } \\
\text { mate) }\end{array}$} & \multicolumn{2}{|c|}{$\begin{array}{l}\text { Actual rope } \\
\text { strength }\end{array}$} & \multicolumn{2}{|c|}{$\begin{array}{l}\text { Approximate aggre- } \\
\text { gate breaking } \\
\text { strength }\end{array}$} \\
\hline $\begin{array}{c}i n . \\
1 / 2 \\
9 / 6 \\
5 / 8 \\
3 / 4 \\
7 / 8\end{array}$ & $\begin{array}{l}m m \\
12.7 \\
14.3 \\
15.9 \\
19.0 \\
22.2\end{array}$ & $\begin{array}{l}i n \\
1^{5} ; 8 \\
1^{33} / 4 \\
2 \\
2^{3 / 8} \\
2^{3} / 4\end{array}$ & $\begin{array}{l}m m . \\
41.3 \\
44.4 \\
50.8 \\
60.3 \\
69.8\end{array}$ & $\begin{array}{r}l b / f t \\
0.22 \\
.28 \\
.34 \\
.49 \\
.63\end{array}$ & $\begin{array}{r}\mathrm{kg} / \mathrm{m} \\
0.33 \\
.42 \\
.50 \\
.73 \\
.94\end{array}$ & $\begin{array}{c}\text { Tons of } \\
2,000 \mathrm{lb} \\
4.0 \\
5.0 \\
6.25 \\
9.0 \\
12.25\end{array}$ & $\begin{array}{c}\text { Tons of } \\
1,000 \mathrm{~kg} \\
3.63 \\
4.53 \\
5.67 \\
8.16 \\
11.1\end{array}$ & $\begin{array}{c}\text { Tons of } \\
2,000 \mathrm{lb} \\
4.44 \\
5.55 \\
6.94 \\
10.0 \\
13.6\end{array}$ & $\begin{array}{c}\text { Tons of } \\
1,000 \mathrm{~kg} \\
4.03 \\
5.03 \\
6.30 \\
9.07 \\
12.3\end{array}$ \\
\hline $\begin{array}{l}1 \\
11 / 8 \\
11 / 4 \\
13 / 8 \\
11 / 2\end{array}$ & $\begin{array}{l}25.4 \\
28.6 \\
31.7 \\
34.9 \\
38.1\end{array}$ & $\begin{array}{l}31 / 8 \\
31 / 2 \\
37 / 8 \\
43 / 8 \\
43 / 4\end{array}$ & $\begin{array}{r}79.4 \\
88.9 \\
98.4 \\
111.0 \\
121.0\end{array}$ & $\begin{array}{r}.88 \\
1.14 \\
1.36 \\
1.66 \\
1.97\end{array}$ & $\begin{array}{l}1.31 \\
1.70 \\
2.02 \\
2.47 \\
2.93\end{array}$ & $\begin{array}{l}15.0 \\
19.0 \\
23.5 \\
28.0 \\
36.0\end{array}$ & $\begin{array}{l}13.6 \\
17.2 \\
21.3 \\
25.4 \\
32.6\end{array}$ & $\begin{array}{l}16.7 \\
21.1 \\
26.1 \\
31.1 \\
40.0\end{array}$ & $\begin{array}{l}15.1 \\
19.1 \\
23.7 \\
28.2 \\
36.2\end{array}$ \\
\hline $\begin{array}{l}15 / 8 \\
13 / 4 \\
17 / 8 \\
2\end{array}$ & $\begin{array}{l}41.3 \\
44.4 \\
47.6 \\
50.8\end{array}$ & $\begin{array}{l}51 / 8 \\
51 / 2 \\
57 / 8 \\
61 / 4\end{array}$ & $\begin{array}{l}130.0 \\
140.0 \\
149.0 \\
159.0\end{array}$ & $\begin{array}{l}2.28 \\
2.67 \\
3.09 \\
3.53\end{array}$ & $\begin{array}{l}3.39 \\
3.97 \\
4.60 \\
5.25\end{array}$ & $\begin{array}{l}42.0 \\
49.0 \\
56.0 \\
60.0\end{array}$ & $\begin{array}{l}38.1 \\
44.4 \\
50.8 \\
54.4\end{array}$ & $\begin{array}{l}46.7 \\
54.4 \\
62.2 \\
66.7\end{array}$ & $\begin{array}{l}42.3 \\
49.3 \\
56.4 \\
60.4\end{array}$ \\
\hline
\end{tabular}


TABLE 31. $6 \times 37$ galvanized steel hawser

\begin{tabular}{|c|c|c|c|c|c|c|c|c|c|c|c|c|c|}
\hline \multirow{2}{*}{\multicolumn{2}{|c|}{$\begin{array}{l}\text { Nominal } \\
\text { rope } \\
\text { diameter }\end{array}$}} & \multirow{2}{*}{\multicolumn{2}{|c|}{$\begin{array}{l}\text { Circumfer- } \\
\text { ence (ap- } \\
\text { proximate) }\end{array}$}} & \multirow{2}{*}{\multicolumn{2}{|c|}{$\begin{array}{l}\text { Weight } \\
\text { (approxi- } \\
\text { mate) }\end{array}$}} & \multicolumn{4}{|c|}{ Actual rope strength } & \multicolumn{4}{|c|}{$\begin{array}{l}\text { Approximate aggregate } \\
\text { breaking strength }\end{array}$} \\
\hline & & & & & & \multicolumn{2}{|c|}{$\begin{array}{l}\text { Improved } \\
\text { plow steel }\end{array}$} & \multicolumn{2}{|c|}{ Plow steel } & \multicolumn{2}{|c|}{$\begin{array}{l}\text { Improved } \\
\text { plow steel }\end{array}$} & \multicolumn{2}{|c|}{ Plow steel } \\
\hline $\begin{array}{c}\text { in. } \\
3.4 \\
13 / 16 \\
7 / 8 \\
1 \\
11 / 16\end{array}$ & $\begin{array}{c}m m \\
19.0 \\
20.6 \\
22.2 \\
25.4 \\
27.0\end{array}$ & $\begin{array}{l}i n . \\
233 \\
21 / 2 \\
234 \\
31 / 8 \\
338\end{array}$ & $\begin{array}{l}m m \\
60.3 \\
63.5 \\
69.8 \\
79.4 \\
85.7\end{array}$ & $\begin{array}{l}l b / f t \\
0.87 \\
1.02 \\
1.19 \\
1.55 \\
1.75\end{array}$ & $\begin{array}{l}k g / m \\
1.29 \\
1.52 \\
1.77 \\
2.31 \\
2.60\end{array}$ & $\begin{array}{c}\text { Tons } \\
\text { of } \\
2,000 \\
l b \\
21.0 \\
24.5 \\
28.4 \\
36.9 \\
41.6\end{array}$ & $\begin{array}{c}\text { Tons } \\
\text { of } \\
1,000 \\
\mathrm{~kg} \\
19.0 \\
22.2 \\
25.8 \\
33.5 \\
37.7\end{array}$ & $\begin{array}{c}\text { Tons } \\
\text { of } \\
2,000 \\
l b \\
18.2 \\
21.3 \\
24.7 \\
32.1 \\
36.1\end{array}$ & $\begin{array}{c}\text { Tons } \\
\text { of } \\
1,000 \\
k g \\
16.5 \\
19.3 \\
22.4 \\
29.1 \\
32.7\end{array}$ & $\begin{array}{c}\text { Tons } \\
\text { of } \\
2,000 \\
l b \\
25.4 \\
29.7 \\
34.4 \\
44.7 \\
50.4\end{array}$ & $\begin{array}{c}\text { Tons } \\
\text { of } \\
1,000 \\
k g \\
23.0 \\
26.9 \\
31.3 \\
40.6 \\
45.7\end{array}$ & $\begin{array}{c}\text { Tons } \\
\text { of } \\
2,000 \\
l b \\
22.1 \\
25.8 \\
29.9 \\
38.9 \\
43.7\end{array}$ & $\begin{array}{c}\text { Tons } \\
\text { of } \\
1,000 \\
k g \\
20.0 \\
23.4 \\
27.1 \\
35.3 \\
39.6\end{array}$ \\
\hline $\begin{array}{l}11 / 8 \\
13116 \\
11 / 4 \\
138 \\
1716\end{array}$ & $\begin{array}{l}28.6 \\
30.2 \\
31.7 \\
34.9 \\
36.5\end{array}$ & $\begin{array}{l}31 / 2 \\
334 \\
378 \\
438 \\
41 / 2\end{array}$ & $\begin{array}{r}88.9 \\
95.2 \\
98.4 \\
111.0 \\
114.0\end{array}$ & $\begin{array}{l}1.96 \\
2.19 \\
2.42 \\
2.93 \\
3.20\end{array}$ & $\begin{array}{l}2.92 \\
3.26 \\
3.60 \\
4.36 \\
4.76\end{array}$ & $\begin{array}{l}46.5 \\
51.7 \\
57.1 \\
68.8 \\
75.0\end{array}$ & $\begin{array}{l}42.2 \\
46.9 \\
51.8 \\
62.4 \\
68.0\end{array}$ & $\begin{array}{l}40.4 \\
44.9 \\
49.7 \\
59.8 \\
65.3\end{array}$ & $\begin{array}{l}36.6 \\
40.7 \\
45.1 \\
54.2 \\
59.2\end{array}$ & $\begin{array}{l}56.4 \\
62.7 \\
69.2 \\
83.4 \\
90.9\end{array}$ & $\begin{array}{l}51.1 \\
56.8 \\
62.8 \\
75.6 \\
82.4\end{array}$ & $\begin{array}{l}49.0 \\
54.4 \\
60.2 \\
72.5 \\
79.1\end{array}$ & $\begin{array}{l}44.4 \\
49.3 \\
54.7 \\
65.7 \\
71.7\end{array}$ \\
\hline $\begin{array}{l}11 / 2 \\
158 \\
1^{111 / 16} \\
134 \\
1^{13 / 16}\end{array}$ & $\begin{array}{l}38.1 \\
41.3 \\
42.9 \\
44.4 \\
46.0\end{array}$ & $\begin{array}{l}43 / 4 \\
5118 \\
51 / 4 \\
51 / 2 \\
5 \% 4\end{array}$ & $\begin{array}{l}121.0 \\
130.0 \\
133.0 \\
140.0 \\
146.0\end{array}$ & $\begin{array}{l}3.49 \\
4.09 \\
4.41 \\
4.75 \\
5.09\end{array}$ & $\begin{array}{l}5.19 \\
6.09 \\
6.56 \\
7.07 \\
7.57\end{array}$ & $\begin{array}{r}81.5 \\
95.3 \\
103.0 \\
110.0 \\
118.0\end{array}$ & $\begin{array}{r}73.9 \\
86.4 \\
93.4 \\
99.8 \\
107.0\end{array}$ & $\begin{array}{r}70.9 \\
82.9 \\
89.2 \\
95.7 \\
102.0\end{array}$ & $\begin{array}{l}64.3 \\
75.2 \\
80.9 \\
86.8 \\
92.5\end{array}$ & $\begin{array}{r}98.8 \\
115.0 \\
125.0 \\
133.0 \\
143.0\end{array}$ & $\begin{array}{r}89.6 \\
105.0 \\
113.0 \\
121.0 \\
130.0\end{array}$ & $\begin{array}{r}85.9 \\
100.0 \\
108.0 \\
116.0 \\
124.0\end{array}$ & $\begin{array}{r}77.9 \\
91.1 \\
98.1 \\
105.0 \\
112.0\end{array}$ \\
\hline $\begin{array}{l}1^{15} / 16 \\
2 \\
21 / 16 \\
21 / 8 \\
21 / 4\end{array}$ & $\begin{array}{l}49.2 \\
50.8 \\
52.4 \\
54.0 \\
57.1\end{array}$ & $\begin{array}{l}61 / 8 \\
61 / 4 \\
61 / 2 \\
6152 \\
71 / 8\end{array}$ & $\begin{array}{l}156.0 \\
159.0 \\
165.0 \\
168.0 \\
181.0\end{array}$ & $\begin{array}{l}5.82 \\
6.20 \\
6.59 \\
7.00 \\
7.85\end{array}$ & $\begin{array}{c}8.66 \\
9.23 \\
9.81 \\
10.4 \\
11.7\end{array}$ & $\begin{array}{l}134.0 \\
143.0 \\
151.0 \\
160.0 \\
179.0\end{array}$ & $\begin{array}{l}121.0 \\
130.0 \\
137.0 \\
145.0 \\
162.0\end{array}$ & $\begin{array}{l}117.0 \\
124.0 \\
132.0 \\
139.0 \\
156.0\end{array}$ & $\begin{array}{l}106.0 \\
112.0 \\
120.0 \\
126.0 \\
141.0\end{array}$ & $\begin{array}{l}162.0 \\
173.0 \\
183.0 \\
194.0 \\
217.0\end{array}$ & $\begin{array}{l}147.0 \\
157.0 \\
166.0 \\
176.0 \\
196.0\end{array}$ & $\begin{array}{l}142.0 \\
150.0 \\
160.0 \\
168.0 \\
189.0\end{array}$ & $\begin{array}{l}128.0 \\
136.0 \\
145.0 \\
153.0 \\
171.0\end{array}$ \\
\hline $\begin{array}{l}25 / 16 \\
238\end{array}$ & $\begin{array}{l}58.7 \\
60.3\end{array}$ & $\begin{array}{l}71 / 4 \\
71 / 2\end{array}$ & $\begin{array}{l}184.0 \\
190.0\end{array}$ & $\begin{array}{l}8.29 \\
8.74\end{array}$ & $\begin{array}{l}12.3 \\
13.0\end{array}$ & $\begin{array}{l}189.0 \\
199.0\end{array}$ & $\begin{array}{l}171.0 \\
180.0\end{array}$ & $\begin{array}{l}164.0 \\
173.0\end{array}$ & $\begin{array}{l}149.0 \\
157.0\end{array}$ & $\begin{array}{l}229.0 \\
241.0\end{array}$ & $\begin{array}{l}207.0 \\
218.0\end{array}$ & $\begin{array}{l}199.0 \\
210.0\end{array}$ & $\begin{array}{l}181.0 \\
190.0\end{array}$ \\
\hline
\end{tabular}

TABLE 32. $6 \times 19$ marline clad grain shovel rope

\begin{tabular}{|c|c|c|c|c|c|c|c|c|c|c|c|}
\hline \multicolumn{4}{|c|}{ Nominal rope diameter } & \multirow{2}{*}{\multicolumn{2}{|c|}{$\begin{array}{l}\text { Circumference } \\
\text { (approximate) }\end{array}$}} & \multirow{2}{*}{\multicolumn{2}{|c|}{$\begin{array}{c}\text { Weight } \\
\text { (approximate) }\end{array}$}} & \multirow{2}{*}{\multicolumn{2}{|c|}{$\begin{array}{c}\text { Actual rope } \\
\text { strength-mild } \\
\text { plow steel }\end{array}$}} & \multirow{2}{*}{\multicolumn{2}{|c|}{$\begin{array}{l}\text { Approximate } \\
\text { aggregate break } \\
\text { ing strength- } \\
\text { mild plow steel }\end{array}$}} \\
\hline Befo & erving & Afte & rving & & & & & & & & \\
\hline $\begin{array}{l}\text { in. } \\
1 / 4 \\
3 / 8\end{array}$ & $\begin{array}{l}m m \\
6.3 \\
9.5\end{array}$ & $\begin{array}{l}\text { in. } \\
5,8 \\
3 / 4\end{array}$ & $\begin{array}{c}m m \\
15.9 \\
19.0\end{array}$ & $\begin{array}{l}\text { in. } \\
2 \\
23 / 8\end{array}$ & $\begin{array}{c}m m \\
50.8 \\
60.3\end{array}$ & $\begin{array}{r}l b / f t \\
0.25 \\
.43\end{array}$ & $\begin{array}{r}\mathrm{kg} / \mathrm{m} \\
0.37 \\
.64\end{array}$ & $\begin{array}{l}\text { Tons of } \\
2,000 \mathrm{lb} \\
2.50 \\
5.50\end{array}$ & $\begin{array}{l}\text { Tons of } \\
1,000 \mathrm{~kg} \\
2.27 \\
4.99\end{array}$ & $\begin{array}{c}\text { Tons of } \\
2,000 \mathrm{lb} \\
2.78 \\
6.11\end{array}$ & $\begin{array}{l}\text { Tons of } \\
1,000 \mathrm{~kg} \\
2.52 \\
5.54\end{array}$ \\
\hline
\end{tabular}

TABLE $33.9 \times 4$ galvanized mast arm rope

\begin{tabular}{|c|c|c|c|c|c|c|c|}
\hline \multicolumn{2}{|c|}{ Nominal rope diameter } & \multicolumn{2}{|c|}{ Weight (approximate) } & \multicolumn{2}{|c|}{ Actual rope strength } & \multicolumn{2}{|c|}{$\begin{array}{l}\text { Approximate aggregate } \\
\text { breaking strength }\end{array}$} \\
\hline $\begin{array}{l}\text { in. } \\
14 \\
5 / 16 \\
3 / 8\end{array}$ & $\begin{array}{l}m m \\
6.3 \\
7.9 \\
9.5\end{array}$ & $\begin{array}{c}l b / f t \\
0.070 \\
.107 \\
.158\end{array}$ & $\begin{array}{c}\mathrm{kg} / \mathrm{m} \\
0.104 \\
.159 \\
.235\end{array}$ & $\begin{array}{c}\text { Tons of } \\
2,000 \text { lb } \\
0.550 \\
.765 \\
1.100\end{array}$ & $\begin{array}{c}\text { Tons of } \\
1,000 \mathrm{~kg} \\
0.499 \\
.694 \\
.998\end{array}$ & $\begin{array}{c}\text { Tons of } \\
2,000 \text { lb } \\
0.639 \\
.889 \\
1.279\end{array}$ & $\begin{array}{c}\text { Tons of } \\
1,000 \mathrm{~kg} \\
0.580 \\
.807 \\
1.160\end{array}$ \\
\hline
\end{tabular}




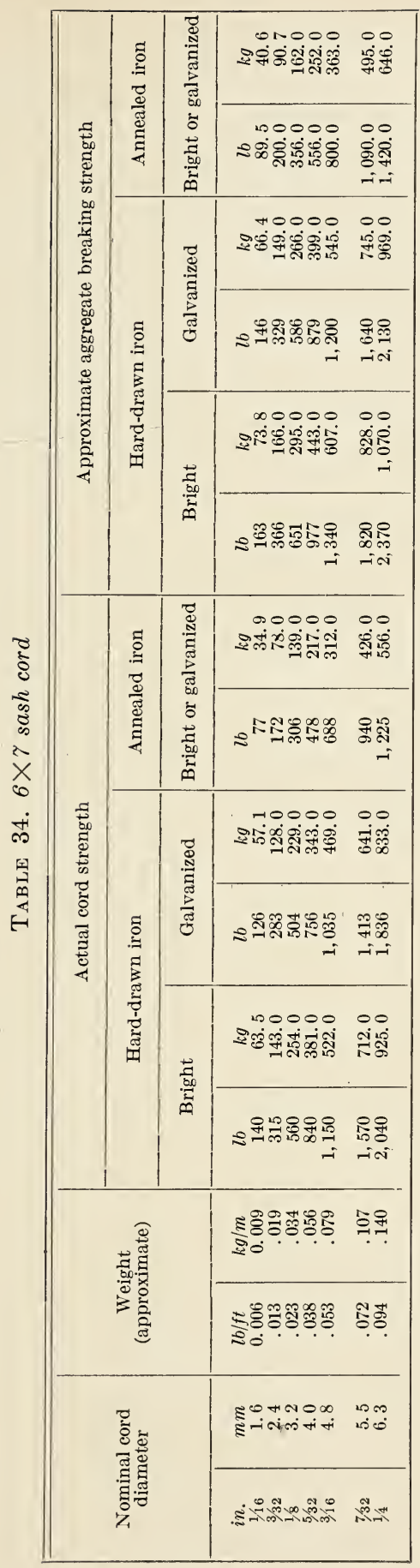


TABLE $35 . \quad 6 \times 7$ aircraft cord

\begin{tabular}{|c|c|c|c|c|c|c|c|}
\hline \multirow{2}{*}{\multicolumn{2}{|c|}{$\begin{array}{l}\text { Nominal cord } \\
\text { diameter }\end{array}$}} & \multirow{2}{*}{\multicolumn{2}{|c|}{ Weight (approximate) }} & \multicolumn{4}{|c|}{ Actual cord strength } \\
\hline & & & & \multicolumn{2}{|c|}{$\begin{array}{l}\text { Carbon steel, tinned } \\
\text { or galvanized }\end{array}$} & \multicolumn{2}{|c|}{$\begin{array}{c}\text { Corrosion-resistant } \\
\text { steel }\end{array}$} \\
\hline $\begin{array}{l}i n . \\
1 / 16 \\
5 ; 64 \\
3 / 32 \\
764 \\
1 / 8\end{array}$ & $\begin{array}{r}m m \\
1.6 \\
2.0 \\
2.4 \\
2.8 \\
3.2\end{array}$ & $\begin{array}{l}l b / 100 \mathrm{ft} \\
0.68 \\
1.0 \\
1.45 \\
2.0 \\
2.55\end{array}$ & $\begin{array}{c}\mathrm{kg} / 100 \mathrm{~m} \\
1.01 \\
1.49 \\
2.16 \\
2.98 \\
3.79\end{array}$ & $\begin{array}{c}\text { Tons of } \\
2,000 \mathrm{lb} \\
0.200 \\
.275 \\
.400 \\
.525 \\
.720\end{array}$ & $\begin{array}{c}\text { Tons of } \\
\text { 1,000 kg } \\
0.181 \\
.249 \\
.363 \\
.476 \\
.653\end{array}$ & $\begin{array}{c}\text { Tons of } \\
2,000 \text { lb } \\
0.192 \\
.260 \\
.400 \\
.505 \\
.700\end{array}$ & $\begin{array}{c}\text { Tons of } \\
1,000 \mathrm{~kg} \\
0.175 \\
.236 \\
.363 \\
.458 \\
.635\end{array}$ \\
\hline $\begin{array}{l}5 / 32 \\
3 / 16 \\
7 / 32 \\
1 / 4 \\
9 / 32\end{array}$ & $\begin{array}{l}4.0 \\
4.9 \\
5.5 \\
6.3 \\
7.1\end{array}$ & $\begin{array}{r}3.9 \\
5.6 \\
7.5 \\
9.7 \\
12.2\end{array}$ & $\begin{array}{c}5.80 \\
8.33 \\
11.2 \\
14.4 \\
18.1\end{array}$ & $\begin{array}{l}1.100 \\
1.575 \\
2.050 \\
2.600 \\
3.300\end{array}$ & $\begin{array}{r}.999 \\
1.429 \\
1.860 \\
2.359 \\
2.994\end{array}$ & $\begin{array}{l}1.050 \\
1.500 \\
2.000 \\
2.600 \\
3.300\end{array}$ & $\begin{array}{r}.952 \\
1.361 \\
1.814 \\
2.359 \\
2.994\end{array}$ \\
\hline $\begin{array}{c}5,16 \\
11 / 32 \\
3 / 8\end{array}$ & $\begin{array}{l}7.9 \\
8.7 \\
9.5\end{array}$ & $\begin{array}{l}15.2 \\
18.2 \\
21.5\end{array}$ & $\begin{array}{l}22.6 \\
27.1 \\
32.0\end{array}$ & $\begin{array}{l}4.000 \\
4.750 \\
5.750\end{array}$ & $\begin{array}{l}\text { 3. } 629 \\
4.309 \\
\text { 5. } 216\end{array}$ & $\begin{array}{l}3.950 \\
4.350 \\
5.500\end{array}$ & $\begin{array}{l}3.583 \\
3.946 \\
4.989\end{array}$ \\
\hline
\end{tabular}

TABLE 36. $\quad 7 \times 7$ aircraft cord

\begin{tabular}{|c|c|c|c|c|c|c|c|}
\hline \multirow{2}{*}{\multicolumn{2}{|c|}{$\begin{array}{l}\text { Nominal cord } \\
\text { diameter }\end{array}$}} & \multirow{2}{*}{\multicolumn{2}{|c|}{ Weight (approximate) }} & \multicolumn{4}{|c|}{ Actual cord strength } \\
\hline & & & & \multicolumn{2}{|c|}{$\begin{array}{l}\text { Carbon steel, tinned } \\
\text { or galvanized }\end{array}$} & \multicolumn{2}{|c|}{$\begin{array}{c}\text { Corrosion-resistant } \\
\text { steel }\end{array}$} \\
\hline $\begin{array}{l}\text { in. } \\
1 / 16 \\
5 / 64 \\
3 / 32 \\
7 / 64 \\
1 / 8\end{array}$ & $\begin{array}{r}m m \\
1.6 \\
2.0 \\
2.4 \\
2.8 \\
3.2\end{array}$ & $\begin{array}{c}l b / 100 f t \\
0.75 \\
1.1 \\
1.6 \\
2.2 \\
2.8\end{array}$ & $\begin{array}{c}\mathrm{kg} / 100 \mathrm{~m} \\
1.12 \\
1.64 \\
2.38 \\
3.27 \\
4.17\end{array}$ & $\begin{array}{c}\text { Tons of } \\
2,000 \mathrm{lb} \\
0.240 \\
.325 \\
.460 \\
.630 \\
.850\end{array}$ & $\begin{array}{c}\text { Tons of } \\
1,000 \mathrm{~kg} \\
0.218 \\
.295 \\
.417 \\
.571 \\
.771\end{array}$ & $\begin{array}{c}\text { Tons of } \\
2,000 \mathrm{lb} \\
0.240 \\
.325 \\
.460 \\
.630 \\
.850\end{array}$ & $\begin{array}{r}\text { Tons of } \\
1,000 \mathrm{~kg} \\
0.218 \\
.295 \\
.417 \\
.571 \\
.771\end{array}$ \\
\hline $\begin{array}{l}5 / 32 \\
3 / 16 \\
7 / 32 \\
1 / 1 \\
9 / 32\end{array}$ & $\begin{array}{l}4.0 \\
4.8 \\
5.5 \\
6.3 \\
7.1\end{array}$ & $\begin{array}{r}4.3 \\
6.2 \\
8.3 \\
10.6 \\
13.4\end{array}$ & $\begin{array}{l}6.40 \\
9.23 \\
12.3 \\
15.8 \\
19.9\end{array}$ & $\begin{array}{l}1.300 \\
1.850 \\
2.400 \\
3.050 \\
3.800\end{array}$ & $\begin{array}{l}1.179 \\
1.678 \\
2.177 \\
2.767 \\
3.447\end{array}$ & $\begin{array}{l}1.300 \\
1.850 \\
2.400 \\
3.050 \\
3.800\end{array}$ & $\begin{array}{l}1.179 \\
1.678 \\
2.177 \\
2.767 \\
3.447\end{array}$ \\
\hline $\begin{array}{c}5 / 16 \\
11 / 32 \\
3 / 8\end{array}$ & $\begin{array}{l}7.9 \\
8.7 \\
9.5\end{array}$ & $\begin{array}{l}16.7 \\
20.1 \\
23.6\end{array}$ & $\begin{array}{l}24.8 \\
29.9 \\
35.1\end{array}$ & $\begin{array}{l}4.600 \\
5.550 \\
6.550\end{array}$ & $\begin{array}{l}\text { 4. } 173 \\
\text { 5. } 035 \\
5.942\end{array}$ & $\begin{array}{l}\text { 4. } 550 \\
5.400 \\
6.300\end{array}$ & $\begin{array}{l}4.128 \\
4.899 \\
5.715\end{array}$ \\
\hline
\end{tabular}

TABLE 37. Galvanized seizing strand $-1 \times 7$

\begin{tabular}{|c|c|c|c|c|c|}
\hline \multicolumn{2}{|c|}{$\begin{array}{l}\text { Nominal strand } \\
\text { diameter }\end{array}$} & \multicolumn{2}{|c|}{ Weight (approximate) } & \multicolumn{2}{|c|}{ Actual strand strength } \\
\hline $\begin{array}{l}\text { in. } \\
1 / 16 \\
3 / 32 \\
1 / 8 \\
5 / 32\end{array}$ & $\begin{array}{l}m m \\
1.6 \\
2.4 \\
3.2 \\
4.0\end{array}$ & $\begin{array}{c}l . b / f t \\
0.010 \\
.020 \\
.033 \\
.050\end{array}$ & $\begin{array}{r}\mathrm{kg} / \mathrm{m} \\
0.015 \\
.030 \\
.049 \\
.074\end{array}$ & $\begin{array}{c}1 b \\
140 \\
300 \\
530 \\
810\end{array}$ & $\begin{array}{r}\mathrm{kg} \\
63.5 \\
136.0 \\
240.0 \\
367.0\end{array}$ \\
\hline
\end{tabular}


TABLE 38. $6 \times 19$ aircraft cord with $7 \times 7$ IWRC

\begin{tabular}{|c|c|c|c|c|c|c|c|}
\hline \multirow{2}{*}{\multicolumn{2}{|c|}{$\begin{array}{l}\text { Nominal cord } \\
\text { diameter }\end{array}$}} & \multirow{2}{*}{\multicolumn{2}{|c|}{ Weight (approximate) }} & \multicolumn{4}{|c|}{ Actual cord strength } \\
\hline & & & & \multicolumn{2}{|c|}{$\begin{array}{l}\text { Carbon steel, tinned } \\
\text { or galvanized }\end{array}$} & \multicolumn{2}{|c|}{$\begin{array}{c}\text { Corrosion-resistant } \\
\text { steel }\end{array}$} \\
\hline $\begin{array}{l}\text { in. } \\
7 / 16 \\
1 / 2 \\
9 / 16 \\
5 / 8 \\
3 / 4\end{array}$ & $\begin{array}{l}m m \\
11.1 \\
12.7 \\
14.3 \\
15.9 \\
19.0\end{array}$ & $\begin{array}{c}l b / 100 f t \\
35.6 \\
45.8 \\
59.0 \\
71.5 \\
105.2\end{array}$ & $\begin{array}{c}\mathrm{kg} / 100 \mathrm{~m} \\
53.0 \\
68.1 \\
87.8 \\
106.4 \\
156.5\end{array}$ & $\begin{array}{c}\text { Tons of } \\
2,000 \mathrm{lb} \\
8.800 \\
11.400 \\
14.250 \\
17.500 \\
24.800\end{array}$ & $\begin{array}{c}\text { Tons of } \\
1,000 \mathrm{~kg} \\
7.983 \\
10.342 \\
12.927 \\
15.876 \\
22.498\end{array}$ & $\begin{array}{c}\text { Tons of } \\
2,000 \mathrm{lb} \\
8.000 \\
11.400 \\
14.250 \\
17.500 \\
24.800\end{array}$ & $\begin{array}{c}\text { Tons of } \\
1,000 \mathrm{~kg} \\
7.257 \\
10.342 \\
12.927 \\
15.876 \\
22.498\end{array}$ \\
\hline $\begin{array}{l}7 / 8 \\
1 \\
11 / 8 \\
11 / 4 \\
13 / 8\end{array}$ & $\begin{array}{l}22.2 \\
25.4 \\
28.6 \\
31.7 \\
34.9\end{array}$ & $\begin{array}{l}143.0 \\
187.0 \\
240.0 \\
290.0 \\
330.0\end{array}$ & $\begin{array}{l}213.0 \\
278.0 \\
357.0 \\
431.0 \\
491.0\end{array}$ & $\begin{array}{l}33.250 \\
42.700 \\
53.200 \\
64.700 \\
76.800\end{array}$ & $\begin{array}{l}30.164 \\
38.737 \\
48.262 \\
58.695 \\
69.672\end{array}$ & $\begin{array}{l}33.250 \\
42.700 \\
53.200 \\
64.700 \\
76.800\end{array}$ & $\begin{array}{l}30.164 \\
38.737 \\
48.262 \\
58.695 \\
69.672\end{array}$ \\
\hline $11 / 2$ & 38.1 & 420.0 & 625.0 & 90.250 & 81.873 & 90.250 & 81.873 \\
\hline
\end{tabular}

TABLE 39. $\quad 7 \times 19$ aircraft cord

\begin{tabular}{|c|c|c|c|c|c|c|c|}
\hline \multirow{2}{*}{\multicolumn{2}{|c|}{$\begin{array}{l}\text { Nominal cord } \\
\text { diameter }\end{array}$}} & \multirow{2}{*}{\multicolumn{2}{|c|}{ Weight (approximate) }} & \multicolumn{4}{|c|}{ Actual cord strength } \\
\hline & & & & \multicolumn{2}{|c|}{$\begin{array}{l}\text { Carbon steel, tinned } \\
\text { or galvanized }\end{array}$} & \multicolumn{2}{|c|}{$\begin{array}{l}\text { Corrosion-resistant } \\
\text { steel }\end{array}$} \\
\hline $\begin{array}{l}\text { in. } \\
1,8 \\
5 / 32 \\
3116 \\
7 / 32 \\
1 / 4\end{array}$ & $\begin{array}{l}m m \\
3.2 \\
4.0 \\
4.8 \\
5.5 \\
6.3\end{array}$ & $\begin{array}{c}l b / 100 \mathrm{ft} \\
2.9 \\
4.5 \\
6.5 \\
8.6 \\
11.0\end{array}$ & $\begin{array}{c}\mathrm{kg} / 100 \mathrm{~m} \\
4.31 \\
6.70 \\
9.67 \\
12.8 \\
16.4\end{array}$ & $\begin{array}{c}\text { Tons of } \\
2,000 \mathrm{lb} \\
1.00 \\
1.40 \\
2.10 \\
2.80 \\
3.50\end{array}$ & $\begin{array}{c}\text { Tons of } \\
1,000 \mathrm{~kg} \\
0.907 \\
1.270 \\
1.905 \\
2.540 \\
3.175\end{array}$ & $\begin{array}{c}\text { Tons of } \\
2,000 \mathrm{lb} \\
0.950 \\
1.30 \\
1.95 \\
2.60 \\
3.30\end{array}$ & $\begin{array}{c}\text { Tons of } \\
1,000 \mathrm{~kg} \\
0.862 \\
1.179 \\
1.769 \\
2.359 \\
2.994\end{array}$ \\
\hline $\begin{array}{l}9 / 32 \\
51 / 6 \\
11 / 32 \\
3 / 8\end{array}$ & $\begin{array}{l}7.1 \\
7.9 \\
8.7 \\
9.5\end{array}$ & $\begin{array}{l}13.9 \\
17.3 \\
20.7 \\
24.3\end{array}$ & $\begin{array}{l}20.7 \\
25.7 \\
30.8 \\
36.2\end{array}$ & $\begin{array}{l}4.00 \\
4.90 \\
6.25 \\
7.20\end{array}$ & $\begin{array}{l}3.629 \\
4.445 \\
5.670 \\
6.532\end{array}$ & $\begin{array}{l}4.00 \\
4.10 \\
6.00\end{array}$ & $\begin{array}{c}3.629 \\
3.719 \\
5.443\end{array}$ \\
\hline
\end{tabular}

TABLE 40. 19-wire aircraft strand

\begin{tabular}{|c|c|c|c|c|c|c|c|}
\hline \multirow{2}{*}{\multicolumn{2}{|c|}{$\begin{array}{l}\text { Nominal strand } \\
\text { diameter }\end{array}$}} & \multirow{2}{*}{\multicolumn{2}{|c|}{ Weight (approximate) }} & \multicolumn{4}{|c|}{ Actual strand strength } \\
\hline & & & & \multicolumn{2}{|c|}{$\begin{array}{l}\text { Carbon steel, tinned } \\
\text { or galvanized }\end{array}$} & \multicolumn{2}{|c|}{$\begin{array}{c}\text { Corrosion-resistant } \\
\text { steel }\end{array}$} \\
\hline $\begin{array}{r}i n . \\
\text { a } 1 / 32 \\
1 / 16 \\
5 / 64 \\
3 / 32 \\
7 / 64\end{array}$ & $\begin{array}{r}m m \\
0.8 \\
1.6 \\
2.0 \\
2.4 \\
2.8\end{array}$ & $\begin{array}{c}l b / 100 \mathrm{ft} \\
0.25 \\
.85 \\
1.4 \\
2.0 \\
2.7\end{array}$ & $\begin{array}{c}\mathrm{kg} / 100 \mathrm{~m} \\
0.37 \\
1.26 \\
2.1 \\
3.0 \\
4.0\end{array}$ & $\begin{array}{c}\text { Tons of } \\
2,000 \mathrm{lb} \\
0.092 \\
.250 \\
.400 \\
.600 \\
.800\end{array}$ & $\begin{array}{c}\text { Tons of } \\
1,000 \mathrm{~kg} \\
0.084 \\
.227 \\
.363 \\
.544 \\
.726\end{array}$ & $\begin{array}{c}\text { Tons of } \\
2,000 \mathrm{lb} \\
0.075 \\
.250 \\
.400 \\
.600 \\
.800\end{array}$ & $\begin{array}{c}\text { Tons of } \\
1,000 \mathrm{~kg} \\
0.068 \\
.227 \\
.363 \\
.544 \\
.726\end{array}$ \\
\hline $\begin{array}{l}1 / 8 \\
5 / 32 \\
3 / 16 \\
7 / 32 \\
1 / 4\end{array}$ & $\begin{array}{l}3.2 \\
4.0 \\
4.8 \\
5.5 \\
6.3\end{array}$ & $\begin{array}{r}3.5 \\
5.5 \\
7.7 \\
10.2 \\
13.5\end{array}$ & $\begin{array}{r}5.2 \\
8.2 \\
11.4 \\
15.2 \\
20.1\end{array}$ & $\begin{array}{l}1.050 \\
1.650 \\
2.350 \\
3.150 \\
4.100\end{array}$ & $\begin{array}{l}.952 \\
1.497 \\
2.332 \\
2.858 \\
3.719\end{array}$ & $\begin{array}{l}1.050 \\
1.650 \\
2.350 \\
3.150 \\
4.100\end{array}$ & $\begin{array}{r}.952 \\
1.497 \\
2.132 \\
2.858 \\
3.719\end{array}$ \\
\hline $\begin{array}{l}9 / 32 \\
5 / 16\end{array}$ & $\begin{array}{l}7.1 \\
7.9\end{array}$ & $\begin{array}{l}17.0 \\
21.0\end{array}$ & $\begin{array}{l}25.3 \\
31.2\end{array}$ & $\begin{array}{l}5.150 \\
6.250\end{array}$ & $\begin{array}{l}\text { 4. } 672 \\
5.670\end{array}$ & $\begin{array}{l}5.150 \\
6.250\end{array}$ & $\begin{array}{l}4.672 \\
5.670\end{array}$ \\
\hline
\end{tabular}

a Made of 7 wires. 
TABLE $41.8 \times 19$ elevator rope

\begin{tabular}{|c|c|c|c|c|c|c|c|c|c|c|c|}
\hline \multirow{2}{*}{\multicolumn{2}{|c|}{$\begin{array}{c}\text { Nominal rope } \\
\text { diameter }\end{array}$}} & \multirow{2}{*}{\multicolumn{2}{|c|}{$\begin{array}{l}\text { Weight } \\
\text { (approxi- } \\
\text { mate) }\end{array}$}} & \multicolumn{4}{|c|}{ Actual rope strength } & \multicolumn{4}{|c|}{$\begin{array}{l}\text { A pproximate aggregate } \\
\text { breaking strength }\end{array}$} \\
\hline & & & & \multicolumn{2}{|c|}{ Traction steel } & \multicolumn{2}{|c|}{ Iron } & \multicolumn{2}{|c|}{ Traction steel } & \multicolumn{2}{|c|}{ Iron } \\
\hline $\begin{array}{l}\text { in. } \\
3 / 16 \\
1 / 4 \\
5 / 16 \\
3 / 8 \\
7 / 16\end{array}$ & $\begin{array}{r}m m \\
4.8 \\
6.3 \\
7.9 \\
9.5 \\
11.1\end{array}$ & $\begin{array}{l}l b / f t \\
0.05 \\
.09 \\
.14 \\
.20 \\
.28\end{array}$ & $\begin{array}{l}\mathrm{kg} / \mathrm{m} \\
0.07 \\
.13 \\
.21 \\
.30 \\
.42\end{array}$ & $\begin{array}{c}\text { Tons of } \\
2,000 \text { lb } \\
1.80 \\
2.80 \\
4.10 \\
5.50\end{array}$ & $\begin{array}{c}\text { Tons of } \\
1,000 \mathrm{~kg} \\
-1.630 \\
2.540 \\
3.720 \\
4.990\end{array}$ & $\begin{array}{c}\text { Tons of } \\
2,000 \mathrm{lb} \\
0.50 \\
.90 \\
1.45 \\
2.10 \\
2.80\end{array}$ & $\begin{array}{l}\text { Tons of } \\
1,000 \mathrm{~kg} \\
0.453 \\
.816 \\
1.310 \\
1.900 \\
2.540\end{array}$ & $\begin{array}{c}\text { Tons of } \\
2,000 \text { lb } \\
-18 \\
2.18 \\
3.39 \\
4.97 \\
6.65\end{array}$ & $\begin{array}{c}\text { Tons of } \\
1,000 \mathrm{~kg} \\
1.970 \\
3.080 \\
4.510 \\
6.050\end{array}$ & $\begin{array}{c}\text { Tons of } \\
2,000 \text { lb } \\
0.605 \\
1.090 \\
1.755 \\
2.545 \\
3.395\end{array}$ & $\begin{array}{c}\text { Tons of } \\
1,000 \mathrm{~kg} \\
0.549 \\
.989 \\
1.590 \\
2.300 \\
3.080\end{array}$ \\
\hline $\begin{array}{l}1 / 2 \\
9 / 16 \\
5 / 8 \\
11 / 16 \\
3 / 1\end{array}$ & $\begin{array}{l}12.7 \\
14.3 \\
15.9 \\
17.5 \\
19.0\end{array}$ & $\begin{array}{l}.36 \\
.46 \\
.57 \\
.69 \\
.82\end{array}$ & $\begin{array}{r}.53 \\
.68 \\
.85 \\
1.03 \\
1.22\end{array}$ & $\begin{array}{r}7.25 \\
9.25 \\
11.50 \\
13.50 \\
16.00\end{array}$ & $\begin{array}{r}6.580 \\
8.390 \\
10.400 \\
12.200 \\
14.500\end{array}$ & $\begin{array}{l}3.60 \\
4.60 \\
5.60 \\
-1.00\end{array}$ & $\begin{array}{l}3.260 \\
4.170 \\
5.080 \\
7.260\end{array}$ & $\begin{array}{r}8.80 \\
11.20 \\
13.95 \\
16.35 \\
19.40\end{array}$ & $\begin{array}{r}7.970 \\
10.200 \\
12.600 \\
14.800 \\
17.600\end{array}$ & $\begin{array}{l}4.365 \\
5.55 \\
6.80 \\
-9.70\end{array}$ & $\begin{array}{l}3.950 \\
5.050 \\
6.160 \\
8.800\end{array}$ \\
\hline $\begin{array}{l}13 / 16 \\
7 / 8 \\
15 / 16 \\
1 \\
11 / 16\end{array}$ & $\begin{array}{l}20.6 \\
22.2 \\
23.8 \\
25.4 \\
27.0\end{array}$ & $\begin{array}{r}.96 \\
1.11 \\
1.27 \\
1.45 \\
1.64\end{array}$ & $\begin{array}{l}1.43 \\
1.65 \\
1.89 \\
2.16 \\
2.44\end{array}$ & $\begin{array}{l}18.50 \\
21.00 \\
24.00 \\
27.00 \\
30.50\end{array}$ & $\begin{array}{l}16.800 \\
19.000 \\
21.800 \\
24.500 \\
27.700\end{array}$ & $\begin{array}{r}10.70 \\
14.00 \\
-.--\end{array}$ & $\begin{array}{r}9.710 \\
12.700 \\
.\end{array}$ & $\begin{array}{l}22.40 \\
25.45 \\
29.10 \\
32.70 \\
36.95\end{array}$ & $\begin{array}{l}20.400 \\
23.000 \\
26.400 \\
29.700 \\
33.600\end{array}$ & $\begin{array}{r}12.9 \overline{9} \\
16.95 \\
-\end{array}$ & $\begin{array}{r}11.800 \\
15.400\end{array}$ \\
\hline
\end{tabular}

TABLE $42.6 \times 19$ elevator rope

\begin{tabular}{|c|c|c|c|c|c|c|c|c|c|c|c|}
\hline \multirow{2}{*}{\multicolumn{2}{|c|}{$\begin{array}{l}\text { Nominal rope } \\
\text { diameter }\end{array}$}} & \multirow{2}{*}{\multicolumn{2}{|c|}{$\begin{array}{l}\text { Weight } \\
\text { (approxi- } \\
\text { mate) }\end{array}$}} & \multicolumn{4}{|c|}{ Actual rope strength } & \multicolumn{4}{|c|}{$\begin{array}{l}\text { A pproximate aggi egate } \\
\text { breaking strength }\end{array}$} \\
\hline & & & & \multicolumn{2}{|c|}{ Traction steel } & \multicolumn{2}{|c|}{ Iron } & \multicolumn{2}{|c|}{ Traction steel } & \multicolumn{2}{|c|}{ Iron } \\
\hline $\begin{array}{l}\text { in. } \\
3 / 16 \\
1 / 4 \\
5 / 16 \\
3 / 8 \\
7 / 16\end{array}$ & $\begin{array}{r}m m \\
4.8 \\
6.3 \\
7.9 \\
9.5 \\
11.1\end{array}$ & $\begin{array}{l}l b / f t \\
0.06 \\
.10 \\
.16 \\
.23 \\
.31\end{array}$ & $\begin{array}{c}\mathrm{kg} / \mathrm{m} \\
0.09 \\
.15 \\
.24 \\
.34 \\
.46\end{array}$ & $\begin{array}{c}\text { Tons of } \\
2,000 \mathrm{lb} \\
1.80 \\
2.80 \\
4.10 \\
5.50\end{array}$ & $\begin{array}{c}\text { Tons of } \\
1,000 \mathrm{~kg} \\
1.630 \\
2.540 \\
3.720 \\
4.990\end{array}$ & $\begin{array}{c}\text { Tons of } \\
2,000 \mathrm{lb} \\
0.65 \\
1.10 \\
1.60 \\
2.50 \\
3.20\end{array}$ & $\begin{array}{c}\text { Tons of } \\
1,000 \mathrm{~kg} \\
0.590 \\
.998 \\
1.450 \\
2.270 \\
2.900\end{array}$ & $\begin{array}{c}\text { Tons of } \\
2,000 \text { lb } \\
2.18 \\
3.39 \\
4.97 \\
6.65\end{array}$ & $\begin{array}{c}\text { Tons of } \\
1,000 \mathrm{~kg} \\
1.970 \\
3.080 \\
4.510 \\
6.050\end{array}$ & $\begin{array}{c}\text { Tons of } \\
2,000 \text { lb } \\
0.78 \\
1.33 \\
1.94 \\
3.03 \\
3.88\end{array}$ & $\begin{array}{l}\text { Tons of } \\
1,000 \mathrm{~kg} \\
0.715 \\
1.210 \\
1.760 \\
2.750 \\
3.510\end{array}$ \\
\hline $\begin{array}{l}1 / 2 \\
9 / 16 \\
5 / 8 \\
11 / 16 \\
3 / 4\end{array}$ & $\begin{array}{l}12.7 \\
14.3 \\
15.9 \\
17.5 \\
19.0\end{array}$ & $\begin{array}{l}.40 \\
.51 \\
.63 \\
.76 \\
.90\end{array}$ & $\begin{array}{r}.59 \\
.76 \\
.94 \\
1.13 \\
1.34\end{array}$ & $\begin{array}{r}7.25 \\
9.25 \\
11.50 \\
13.50 \\
16.00\end{array}$ & $\begin{array}{r}6.580 \\
8.390 \\
10.400 \\
12.200 \\
14.500\end{array}$ & $\begin{array}{l}4.20 \\
5.30 \\
6.40 \\
9.10\end{array}$ & $\begin{array}{l}3.810 \\
4.810 \\
5.800 \\
-8.250\end{array}$ & $\begin{array}{r}8.80 \\
11.20 \\
13.95 \\
16.35 \\
19.40\end{array}$ & $\begin{array}{r}7.970 \\
10.200 \\
12.600 \\
14.800 \\
17.600\end{array}$ & $\begin{array}{r}5.10 \\
6.40 \\
7.75 \\
11.05\end{array}$ & $\begin{array}{r}4.620 \\
5.830 \\
7.030 \\
10.000\end{array}$ \\
\hline $\begin{array}{l}13 / 16 \\
7 / 8 \\
15 / 16 \\
1 \\
11 / 16\end{array}$ & $\begin{array}{l}20.6 \\
22.2 \\
23.8 \\
25.4 \\
27.0\end{array}$ & $\begin{array}{l}1.06 \\
1.23 \\
1.41 \\
1.60 \\
1.81\end{array}$ & $\begin{array}{l}1.58 \\
1.83 \\
2.10 \\
2.38 \\
2.69\end{array}$ & $\begin{array}{l}18.50 \\
21.00 \\
24.00 \\
27.00 \\
30.50\end{array}$ & $\begin{array}{l}16.800 \\
19.000 \\
21.800 \\
24.500 \\
27.700\end{array}$ & $\begin{array}{r}12.40 \\
16.00 \\
-.-\end{array}$ & $\begin{array}{c}11.200 \\
14.500 \\
-\end{array}$ & $\begin{array}{l}22.40 \\
25.45 \\
29.10 \\
32.70 \\
36.95\end{array}$ & $\begin{array}{l}20.400 \\
23.000 \\
26.400 \\
29.700 \\
33.600\end{array}$ & $\begin{array}{r}15.05 \\
19.40 \\
-.-\end{array}$ & $\begin{array}{r}13.600 \\
17.600 \\
-. .\end{array}$ \\
\hline
\end{tabular}

\section{GENERAL RECOMMENDATIONS}

19. Sizes of sheaves and drums for wire rope.

19a. For the proper serviceability of wire rope, care should be taken that sheaves and drums are of sufficient diameter to give most economical service.

19b. The recommended diameters of drums and sheaves for various sizes of wire rope are given in table 43 . 'The minimum diameters, below which it would be inadvisable to operate, are also shown. Larger sheave and drum diameters than those shown in the table increase rope life and contribute to more economical service. 
TABLE 43. Recommended sheave and drum diameters for wire rope

\begin{tabular}{|c|c|c|}
\hline \multirow{2}{*}{ Wire rope construction } & \multicolumn{2}{|c|}{ Diameter of drum and sheave } \\
\hline & Recommended & Minimum \\
\hline $\begin{array}{l}6 \times 7 \text { rope } \\
6 \times 19 \text { classification rope } \\
6 \times 25 \text { rope (type B flattened strand) } \\
6 \times 30 \text { rope (type } \mathrm{G} \text { flattened strand) } \\
6 \times 37 \text { classification rope } \\
8 \times 19 \text { classification rope } \\
18 \times 7 \text { rope }\end{array}$ & $\begin{array}{l}72 \text { times rope diam. } \\
45 \text { times rope diam. } \\
45 \text { times rope diam. } \\
45 \text { times rope diam. } \\
27 \text { times rope diam. } \\
31 \text { times rope diam. } \\
51 \text { times rope diam. }\end{array}$ & $\begin{array}{l}42 \text { times rope diam. } \\
30 \text { times rope diam. } \\
30 \text { times rope diam. } \\
30 \text { times rope diam. } \\
18 \text { times rope diam. } \\
21 \text { times rope diam. } \\
34 \text { times rope diam. }\end{array}$ \\
\hline
\end{tabular}

20. Stresses due to acceleration.-Stresses in wire rope due to acceleration must be considered when used on mine shaft hoists, inclines, and elevators. In the particular case of elevators most safety codes require factors of safety, based on static loading, high enough to cover accelerating loads without special consideration. Where it is necessary to calculate the stress due to acceleration, the following formula can be used:

$$
M=\frac{W}{g}, \quad a=\frac{V}{t}, \quad F=M a=\frac{W V}{g t}
$$

where

English

$(\mathrm{ft} / \mathrm{sec}) / \mathrm{sec}$

$1 \mathrm{~b}$

$(32.2 \mathrm{ft} / \mathrm{sec}) / \mathrm{sec}$

sec

$\mathrm{ft} / \mathrm{sec}$

$\mathrm{lb}$
Metric

$(M /$ sec $) / \sec$

$\mathrm{kg}$

(9.806 $\mathrm{M} / \mathrm{sec}) / \mathrm{sec}$

sec

$M /$ sec

$\mathrm{kg}$

21. Working loads for wire rope.-In determining the maximum safe working load for a wire rope, it has been considered advisable not to subject the rope to a load greater than one-fifth of the actual breaking strength of the particular rope, or a safety factor of 5. Tables 6 and 6 a show the minimum safety factors recommended for various types of uses. Higher safety factors are often required for safe and economical operation.

21a. The proper factor of safety for a wire rope should be determined by careful and thorough consideration of all pertinent data. Such data should include all loads, acceleration, deceleration, rope speed, attachments, the number, size, and arrangement of sheaves and drums, existing conditions causing corrosion and abrasion, length of rope required, economical life, and degree of danger to life and property.

$21 \mathrm{~b}$. Sometimes it is difficult to determine the load on a wire rope, in which case it can often be figured approximately from the horsepower rating of the motor or engine. The following formula can be used:

\section{Load on rope $=\frac{\text { Horsepower } \times \text { time in seconds } \times \text { horsepower factor }}{\text { Distance }}$.}

The result obtained from the formula should be increased by the permissible or possible overload of the driving unit. 
22. Groove, sheave.-The grooves in sheaves should be slightly larger than the rope to avoid pinching and binding and to permit the rope to adjust itself readily to the radius of curvature. The diameter of a new rope may exceed the nominal diameter by the amounts shown in table 4, tolerances; and the recommended minimum, new, and remachined tolerances for sheave groove diameters above the nominal rope diameter are shown in table 44 . Groove diameters larger than those indicated will not properly support the rope and are not recommended.

TABLE 44. Tolerances by which groove diameter should exceed nominal rope diameter

\begin{tabular}{|c|c|c|c|c|c|}
\hline \multicolumn{2}{|c|}{ Nominal rope diameter } & \multicolumn{2}{|c|}{ Minimum } & \multicolumn{2}{|c|}{$\begin{array}{l}\text { Recommended new } \\
\text { or remachined }\end{array}$} \\
\hline $\begin{array}{l}1 / 4 \text { to } 5 / 16 \\
3,8 \text { to } 3 / 414 \\
13 / 16 \text { to } 11 / 8 \\
13116 \text { to } 11 / 2 \\
1916 \text { to } 21 / 4 \\
25 / 16 \text { and } u p\end{array}$ & $\begin{array}{l}6.3 \text { to } 7.9 \\
9.5 \text { to } 19.0 \\
20.6 \text { to } 28.6 \\
30.2 \text { to } 38.1 \\
39.7 \text { to } 57.2 \\
58.7 \text { and up }\end{array}$ & $\begin{array}{l}\text { in. } \\
1 / 64 \\
1 / 32 \\
3 / 64 \\
1 / 16 \\
3 / 32 \\
1 / 8\end{array}$ & $\begin{array}{r}m m \\
0.40 \\
.79 \\
1.19 \\
1.59 \\
2.38 \\
3.18\end{array}$ & $\begin{array}{l}\text { in } \\
1 / 32 \\
1 / 16 \\
3 / 32 \\
1 / 8 \\
3 / 16 \\
1 / 4\end{array}$ & $\begin{array}{l}m m \\
0.79 \\
1.59 \\
2.38 \\
3.18 \\
4.76 \\
6.35\end{array}$ \\
\hline
\end{tabular}

23. Figure 4 shows the tread diameter, groove diameter, and the over-all diameter of the sheave.

24. Fleet angle of drums.

24a. Where a wire rope leads over a sheave and onto a drum, the rope will not remain in alinement with the sheave groove but will deviate to either side, depending on the width of the drum and its distance from the first fixed sheave. The angle between the center line through the sheave and the center line of the rope leading to the drum is called the fleet angle. (See fig. 5.)

$24 \mathrm{~b}$. To a void excessive wear to the sheave and to prevent excessive chafing of the oncoming wire rope against previous wraps on the drum, it is desirable to keep the fleet angle as small as possible. It is considered good practice to keep the fleet angle to not over $1 \frac{1}{2}$ degrees, which is equivalent to a distance of 38 feet $(11.6 \mathrm{~m})$ of lead from sheave to drum for each foot of drum width either side of the center line of sheave. Where space limitations are not restricted, such as on many mine hoists, the fleet angle is sometimes as small as $1 / 2$ degree.

25. Spooling.

25a. The method described below may be used to determine the proper direction of rope lay for spooling or winding on flat- or smoothfaced drums.

$25 \mathrm{~b}$. When a rope is wound onto a drum any tendency of the rope to twist when tension is released will be in a direction which would untwist the rope at the free end.

$25 \mathrm{c}$. The advantage in applying rope of proper direction of lay is that when the load is slacked off, the several coils on the drum will hug together and maintain an even layer. With rope of improper lay the coils will spread apart at each removal of load and when winding is resumed the rope may criss-cross and overlap on the drum 
with flattening and crushing of the rope as a result. The proper direction of rope lay for either regular or lang lay rope, to give best results, is shown in figure 6 .

25d. On new equipment and also on existing equipment, where possible, the drum anchorage should be so positioned as to favor rightlay rope, since left-lay rope is not always available from stock.

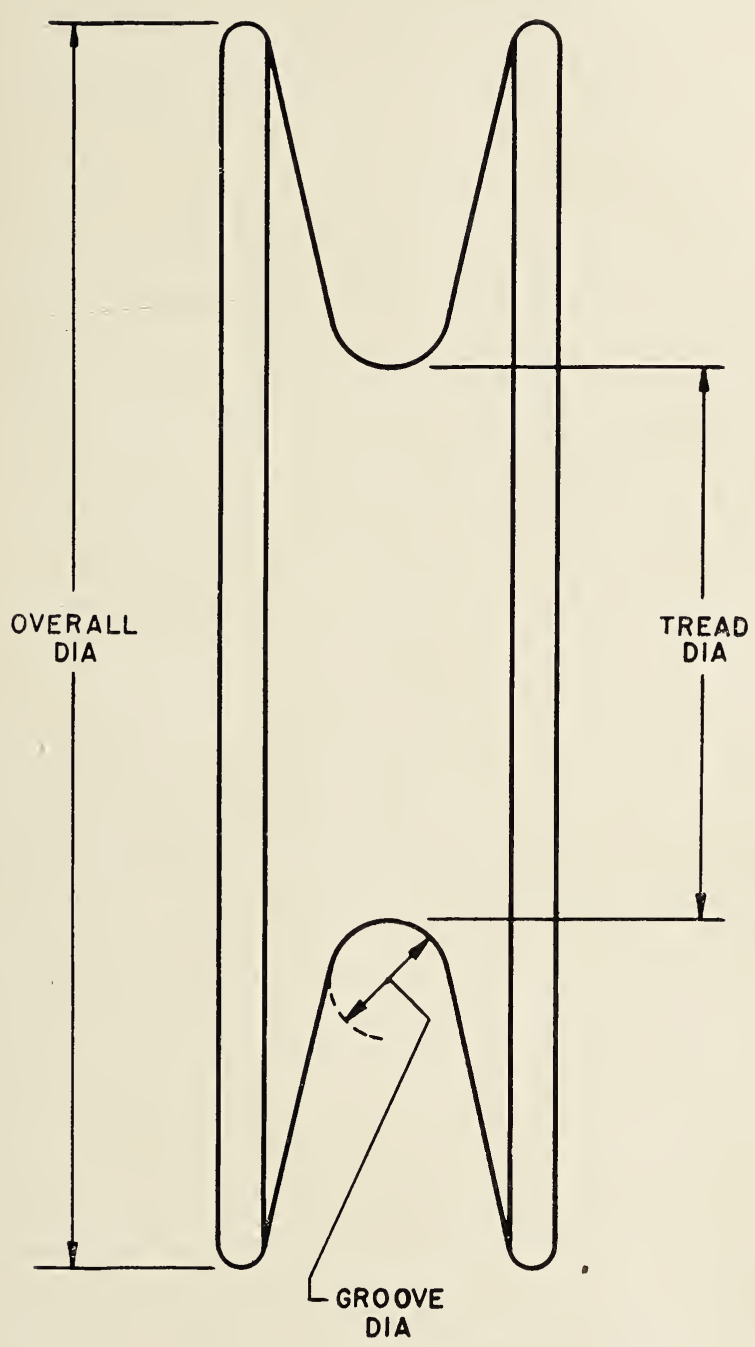

Figure 4. Sheaves.

26. Drum and reel capacities.

$26 \mathrm{a}$. Often it is necessary to determine the amount of rope which can be wound on a drum or reel. 


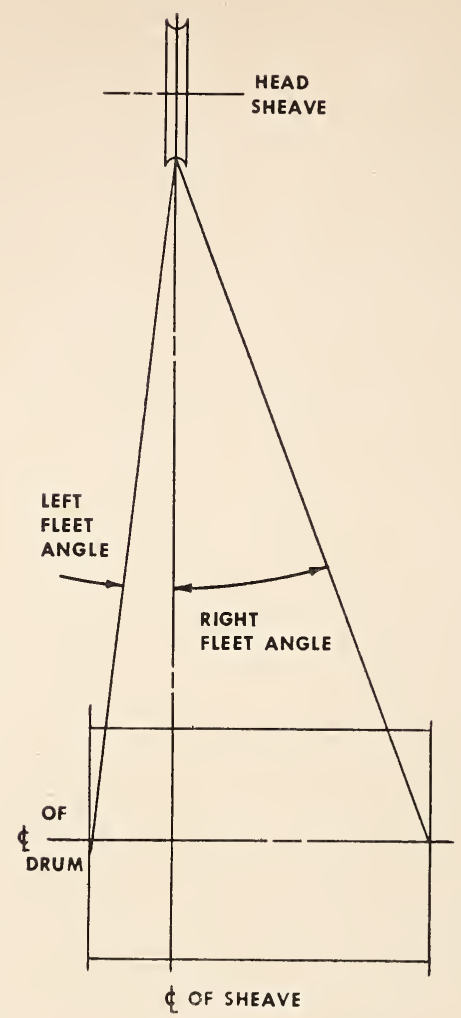

Figure 5. Fleet angle of drums.

Figure 7 shows the method used for measurement of a drum or reel. 'The capacity of the reel or' drum may be calculated as follows, where values for the constants may be found in table 45 .

\section{English units}

$L=(A+D) \times A \times B \times K$.

$L=$ Rope length, in feet.

$K=$ Constant shown below.

$A, B, D$ are in inches.

\section{Metric units}

$L=(A+D) \times A \times B \times C$.

$L=$ Rope length, in meters.

$C=$ Constant shown below.

$A, B, D$ are in centimeters.

26b. Values of $K$ and $C$ allow for normal oversize of ropes. Clearance shown on sketch should usually be 2 inches $(5 \mathrm{~cm})$ unless fittings on ends of rope require greater clearance.

26c. The formula is based on uniform rope winding and will not give correct results if rope is wound nonuniformly on the reel. It is based on the same number of wraps of rope in each layer, which is not strictly correct, but which does not result in appreciable error unless the transverse $(B)$ of the reel is quite small compared with the flange diameter $(H)$. 

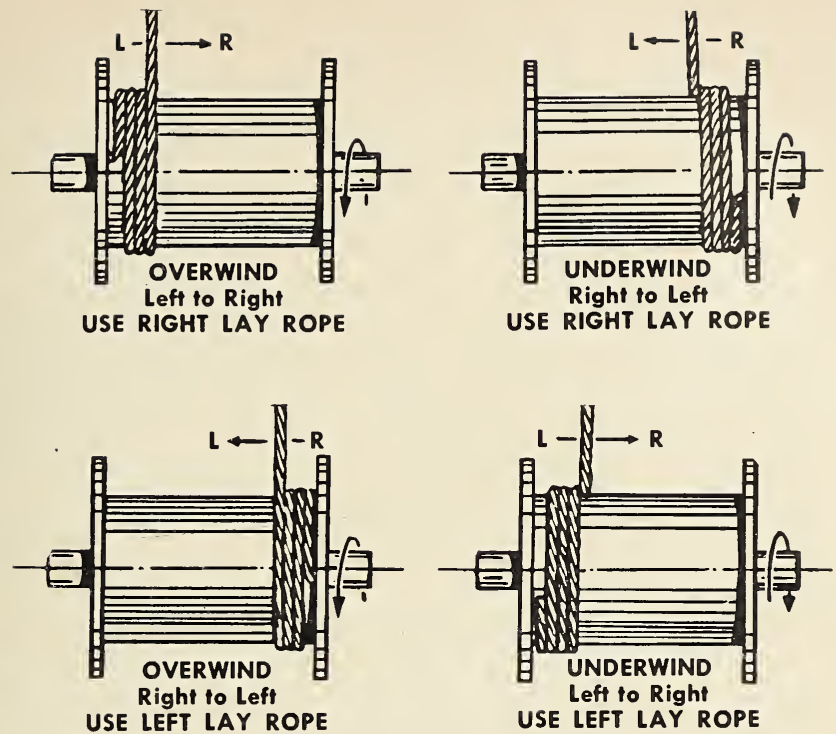

USE LEFT LAY ROPE

Figure 6. Rope lays on drums.

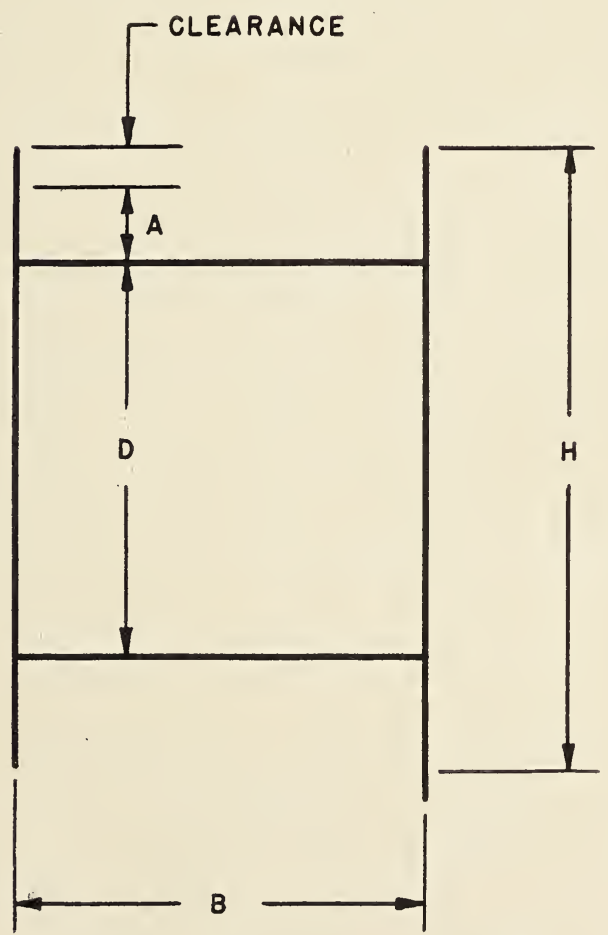

Figure 7. Drum and reel capacities. 
TABLE 45. Constants to determine drum and reel capacities

\begin{tabular}{|c|c|c|c|c|c|c|c|}
\hline \multicolumn{2}{|c|}{ Rope diameter } & \multicolumn{2}{|c|}{ Constant } & \multicolumn{2}{|c|}{ Rope diameter } & \multicolumn{2}{|c|}{ Constant } \\
\hline $\begin{array}{l}\text { in. } \\
1 / 16 \\
3 / 32 \\
1 / 8 \\
5 / 32 \\
3 / 16\end{array}$ & $\begin{array}{r}m m \\
1.6 \\
2.4 \\
3.2 \\
4.0 \\
4.8\end{array}$ & $\begin{array}{c}K \\
49.8 \\
23.4 \\
13.6 \\
8.72 \\
6.14\end{array}$ & $\begin{array}{r}C \\
0.926 \\
.435 \\
.253 \\
.162 \\
.114\end{array}$ & $\begin{array}{c}i n . \\
13 / 16 \\
7 / 8 \\
1 \\
11 / 8 \\
11 / 4\end{array}$ & $\begin{array}{l}m m \\
20.6 \\
22.2 \\
25.4 \\
28.6 \\
31.7\end{array}$ & $\begin{array}{c}K \\
0.354 \\
.308 \\
.239 \\
.191 \\
.152\end{array}$ & $\begin{array}{c}C \\
0.00658 \\
.00573 \\
.00444 \\
.00355 \\
.00283\end{array}$ \\
\hline $\begin{array}{l}7 / 32 \\
1 / 4 \\
5 / 16 \\
3 / 8 \\
7 / 16\end{array}$ & $\begin{array}{r}5.5 \\
6.3 \\
7.9 \\
9.5 \\
11.1\end{array}$ & $\begin{array}{l}4.59 \\
3.29 \\
2.21 \\
1.58 \\
1.19\end{array}$ & $\begin{array}{l}.0854 \\
.0612 \\
.0411 \\
.0294 \\
.0221\end{array}$ & $\begin{array}{l}13 / 8 \\
11 / 2 \\
15 / 8 \\
13 / 4 \\
178\end{array}$ & $\begin{array}{l}34.9 \\
38.1 \\
41.3 \\
44.4 \\
47.6\end{array}$ & $\begin{array}{l}.127 \\
.107 \\
.0886 \\
.0770 \\
.0675\end{array}$ & $\begin{array}{l}.00236 \\
.00199 \\
.00165 \\
.00143 \\
.00125\end{array}$ \\
\hline $\begin{array}{c}1 / 2 \\
9 / 16 \\
5 / 8 \\
11 / 16 \\
3 / 4\end{array}$ & $\begin{array}{l}12.7 \\
14.3 \\
15.9 \\
17.5 \\
19.0\end{array}$ & $\begin{array}{r}0.925 \\
.741 \\
.607 \\
.506 \\
.428\end{array}$ & $\begin{array}{l}.0172 \\
.0138 \\
.0113 \\
.00941 \\
.00796\end{array}$ & $\begin{array}{l}2 \\
21 / 8 \\
21 / 4 \\
23 / 8 \\
21 / 2\end{array}$ & $\begin{array}{l}50.8 \\
54.0 \\
57.1 \\
60.3 \\
63.5\end{array}$ & $\begin{array}{l}.0597 \\
.0532 \\
.0476 \\
.0419 \\
.0380\end{array}$ & $\begin{array}{l}.00111 \\
.000989 \\
.000885 \\
.000779 \\
.000707\end{array}$ \\
\hline
\end{tabular}

\section{Socketing.}

27a. Sockets made of forged steel may be attached to wire rope to make the rope serviceable for certain operations. The proper application of the socket is important and the following instructions should be followed except for socketing elevator ropes when the proper method of established elevator codes should be followed.

$27 \mathrm{~b}$. The wire rope should be securely seized before cutting and not less than two additional seizings placed at a distance from the end equal to the length of the basket of the socket. For large ropes the seizing should be several inches long and securely wrapped with a seizing iron. This operation is very important as it prevents untwisting of the rope and insures equal tension in all the strands when the load is applied.

$27 \mathrm{c}$. The end seizing on the wire rope should be taken off, leaving the additional seizing at a distance from the end equal to the length of the socket basket. The strands can be separated and the fiber core should be cut back to this seizing. All the wires should be untwisted and "broomed out," although they need not be straightened.

$27 \mathrm{~d}$. The wires, for the distance that they are to be inserted in the socket, should be carefully cleaned with benzine, naphtha, or gasoline. For a distance not more than three-quarters of the cleaned length, the wires should then be dipped in commercial muriatic acid for from 30 seconds to 1 minute, or until the acid has thoroughly cleaned each wire. Care should be taken that the acid does not come into contact with any other portion of the rope, for the acid will cause the wires to become brittle.

27 e. The wires should be dipped in boiling water.

$27 \mathrm{f}$. The wires should then be inserted into the basket of the socket, which has been heated to hand heat. Care should be taken that the socket is securely held in line with the axis of the wire rope.

$27 \mathrm{~g}$. The base of the socket should be sealed with putty, clay, or any similar substance, and molten zinc poured into the basket until it is full.

$27 \mathrm{~h}$. The zinc should not be too hot or it will anneal the wires, particularly those in small wire ropes. The temperature should be not more than $830^{\circ} \mathrm{F}\left(443.3^{\circ} \mathrm{C}\right)$. If the zinc is at the proper temperature 
a soft pine stick dipped into the zinc and quickly withdrawn should not have any zinc adhering to it, and it should be only slightly discolored by scorching. If the zinc adheres to the stick, it is too cold for pouring. If the stick is charred, the zinc is too hot.

27i. When the zinc has solidified sufficiently the socket may be plunged into cold water. If a specimen for tensile test is properly socketed, the strands will break near the middle of the wire rope and not at the socket.

28. Lubrication.-Wear of a running wire rope occurs where the outside wires come into contact with the sheaves and drums, especially if slipping takes place, and where the wires are in contact with each other. During fabrication of a rope, the fiber core is saturated with lubricating compound, which in service is gradually supplied to the wires and reduces the wear on them. As the core will not carry enough lubricant for the life of the rope, it is necessary occasionally to apply a lubricant to the outside of the rope. Some of the lubricant will penetrate the rope and be absorbed by the core.

\section{MARKING AND CERTIFICATION}

29. Identification:-Each shipment of wire rope shall have clearly marked on the container the name and address of the manufacturer, together with length, diameter, construction, grade, core, and whether the rope is preformed or nonpreformed.

30. Certification.- In order that purchasers may be assured that wire rope purchased actually complies with the requirements of this commercial standard, it is recommended that manufacturers include the following statement in conjunction with their name and address on labels, invoices, sales literature, etc.:

This wire rope complies with Commercial Standard CS154E-49, developed by the trade, under the procedure of the National Bureau of Standards and issued by the U. S. Department of Commerce.

\section{GLOSSARY OF TECHNICAL TERMS}

31. The following is a glossary of technical terms and definitions peculiar to the wire rope industry for export trade.

Abrasion.-Surface wear on the wires of a wire rope.

Acceleration stress.-Additional stress imposed on a wire rope due to increasing velocity of load.

Aerial conveyor.-See Cableway and Tramway.

Aircraft cables.- Strands, cords, and wire ropes made of special strength wire primarily for aircraft controls and miscellaneous uses of the aircraft industry.

Albert's lay. - Synonymous with Lang lay.

Alternate lay.-Lay of a wire rope in which the strands are alternately regular and lang lay.

Area, metallic.-Sum of the cross-sectional areas of individual wires in a wire rope or strand.

Armored rope.-See Steel clad rope. 
Back-stay.-Guy used to support a boom or mast; or that section of a main cable, as on a suspension bridge, cableway, etc., leading from the tower to the anchorage.

Bail.-A U-shaped member of a bucket, socket, or other fitting.

Bailing line.-The wire rope which operates the bailer for removing water and drill cuttings in drilling a well.

Barney car.-A relatively small car permanently attached to a haulage rope for pushing cars along a haulage system.

Basket of socket.-The conical portion of a socket into which a splayed rope end is inserted and secured with zinc.

Becket loop.-A loop of small rope or strand fastened to the end of a large wire rope to facilitate installation.

Bending stress.- Stress imposed on wires of a wire rope by bending. This stress need not be added to direct load stresses. When sheaves and drums are of suitable size it does not affect the normal life of the wire rope.

Boom line.-A wire rope for supporting or operating the boom of derricks, cranes, drag lines, shovels, etc.

Breaking strength (wire rope).-The measured load required to break a wire rope in tension.

Breaking strength (aggregate wire).-The sum of the breaking strengths in tension of all the wires of a wire rope when the wires are tested individually.

Bridge cable.-The all-metallic wire ropes or strands used as the catenary and suspenders on a suspension bridge.

Bridge socket. - Steel castings with baskets for securing rope ends and equipped with adjustable bolts. Closed type has U-bolt. Open type has two eyebolts and pin.

Bridle cable.-A two-part wire-rope sling attached to a single-part line. The legs of the sling are spread to divide and equalize the load.

Bright rope.-Wire rope made of wires that are not coated with zinc or tin.

Bronze ropes.-Wire rope made of bronze wires.

Button conveyor rope.-Wire ropes to which buttons or disks are attached at regular intervals to move material in a trough.

Cable.-A term loosely applied to wire ropes, wire strands, manila ropes, and electrical conductors.

Cable-laid wire rope.-A type of wire rope consisting of several wire ropes laid into a single-wire rope. Example, $6 \times 6 \times 7$ tiller rope.

Cable crowd rope.-A wire rope used to force the bucket of a power shovel into material handled.

Cable tool drilling line.-The wire rope used to operate the cutting tools in drilling by the "standard" or "cable tool" method.

Cableway.-Aerial conveying system for transporting single loads along a suspended track cable.

Casing line.-Wire rope used to install the casing in an oil well. Synonymous with rotary drilling line in some fields.

Centers.-Wire, strand, or fiber in the center of a strand about which the wires are laid.

Choker rope. - Short wire rope sling used to form a slip noose around the object to be moved or lifted. 
Circumference.-Measured perimeter of a circle circumscribing the wires of a strand or the strands of a wire rope.

Clamps, strand.-A fitting for forming a loop at the end of a length of strand, consisting of two grooved plates and bolts.

Cleaning-out line.-Wire rope used with tools for cleaning out the bottom of an oil well.

Clevis.-See Shackle.

Clip.-Fitting for clamping two parts of wire rope.

Closed socket.-Wire rope and fitting consisting of basket and bail made integral.

Closing line.-Wire rope that closes a clamshell or orange peel bucket and then operates as a hoisting rope.

Coarse laid rope.-Term used in oil fields to designate a $6 \times 7$ wire rope.

Coil.-Circular bundle of wire rope not packed on a reel.

Come-along.-Device for making a temporary grip on a wire rope.

Common strand.-Galvanized strand made of galvanized-iron wire. See Grades, strand.

Conical drum.-Grooved hoisting drum of varying diameter.

Construction.-Design of wire rope, including number of strands, number of wires per strand, and arrangement of wires in each strand.

Continuous bend.-Reeving of wire rope over sheaves and drums so that it bends in one direction, as opposed to reverse bend.

Conveyor rope.-Parallel endless wire ropes used to carry material. See also Button conveyor rope.

Cord.- Term applied to small sizes of wire ropes.

Core.-Core member of a wire rope about which the strands are laid. It may be fiber, a wire strand, or an independent wire rope.

Coring line.-Wire rope used to operate the coring tool for taking core samples during the drilling of a well.

Corrosion.-Chemical decomposition of the wires of a rope by exposure to moisture, acids, alkalies, or other destructive agents.

Corrosion-resistant steel.-Wire rope made of chrome-nickel steel wires having great resistance to corrosion.

Corrugated.-Term used to describe the grooves of a sheave or drum when worn so as to show the impression of a wire rope.

Cotton center.- See Fiber centers.

Cotton core.-See Fiber cores.

Coupling (track strand).-Device for joining the ends of two lengths of track strand.

Cover wires.-Outer layer of wires.

Cracker.-Manila rope spliced to the end of a wire-rope drilling line.

Critical diameter.-Diameter of the smallest bend for a given wire rope which permits the wires and strands to adjust themselves by relative movement while remaining in their normal position.

Crowd rope.- See Cable crowd rope.

Cylindrical drum.- - Hoisting drum of uniform diameter.

Dead line.-An endless-wire rope used in drilling for suspending the casing block from the crown block.

Deceleration stress.-Additional stress imposed on a wire rope due to decreasing the velocity of the load. 
Deflection.-(a) Sag of a rope in a span. Usually measured at midspan as the depth from the chord joining the tops of the two supports. (b) Any deviation from a straight line.

Diameter.-Distance measured across the center of a circle circumscribing the wires of a strand or the strands of a wire rope.

Dog-leg.-Permanent short bend or kink in a wire rope caused by improper use.

Dragline.-Wire rope used to pull an excavating or drag bucket.

Drilling line.-See Cable tool drilling line and Rotary lines.

Drum.-A cylindrical flanged barrel of uniform or tapering diameter on which rope is wound for operation or storage. It may be smooth or grooved.

Efficiency (wire rope).-Percentage ratio of measured breaking strength of a wire rope to the aggregate strength of all individual wires tested separately.

Elastic limit.-Limit of stress above which a permanent deformation takes place within the material. This limit is approximately 55 to 65 percent of breaking strength of steel wire ropes.

Endless rope.-Rope whose two ends are spliced together.

Equalizing slings.-Slings composed of wire rope and equalizing fittings.

Equalizing thimbles.- Special type of fitting used as a component part of some wire rope slings.

Extra-flexible wire rope.-Term used in describing either $8 \times 19$ or $6 \times 37$ constructions of wire rope.

Extra-high-strength strand.-A grade of galvanized or bright strand.

Extra-pliable wire rope.- See Extra flexible wire rope.

Eye or eye splice. - A loop with or without a thimble formed in the end of a wire rope.

Factor of safety.-Ratio of breaking strength of a wire rope to total working load.

Fall rope.-Wire rope in the falls or tackle.

Fatigue.-Term commonly applied to progressive fracture of wires of a rope.

Ferry rope.-Wire rope suspended over water to guide a boat.

Fiber centers.-Cords or rope made of vegetable fiber used in center of a strand.

Fiber cores.-Cords or rope made of vegetable fiber used in core of a wire rope.

Filler wire.-Small auxiliary wires in a strand for spacing and positioning other wires.

Fitting.-Any accessory used as an attachment for wire rope.

Flag.-Marker on a rope to designate position of load.

Flat rope.-Wire rope made of parallel alternating right-lay and leftlay ropes sewn together by relatively soft wires.

Flattened strand rope.-A wire rope, with either oval- or triangularshaped strands, which presents a flattened rope surface.

Fleet angle.-Angle between position of a rope at the extreme end wrap on a drum, and a line drawn perpendicular to the axis of the drum through the center of the nearest fixed sheave.

Flexible-wire rope.-Term used in describing $6 \times 19$ constructions of wire rope.

Galvanize.-To coat with zinc to protect against corrosion. 
Galvanized rope.-Rope made of galvanized wire.

Galvanized strand.- Strand made of galvanized wire.

Galvanized wire.-Wire coated with zinc.

Grades, rope.-Classification of wire rope by its breaking strength. In order of increasing breaking strengths they are iron, traction, mild plow steel, plow steel, improved plow steel.

Grades, strand.-Classification of strand by its breaking strength. In order of increasing breaking strengths they are common, SiemensMartin, high strength and extra-high strength. A utilities grade strand is also made to meet special requirements.

Grain shovel rope.-6×19 marline clad rope used for handling grain in scoops.

Grommet.-An endless seven-strand wire rope made from one continuous length of strand.

Grooved drum.-Drum with grooved surface to accommodate and guide the rope.

Grooves.-Depressions in the periphery of a sheave or drum for positioning and supporting a rope.

Guard rail cable.-A galvanized wire rope or strand erected along a highway.

Guy line.-Strand or rope, usually galvanized, for holding a structure in position.

Haulage rope.-Wire rope used for pulling cars on a track.

Hawser.-Wire rope, usually galvanized, used for towing or mooring vessels.

High-strength strand.-Grade of galvanized or bright strand.

Highway guard cable.-See Guard rail cable.

Holding line.-Wire rope on a clamshell or orange peel bucket that holds the bucket while the closing line is released to dump the load.

Idler.-Sheave or roller used to guide or support a rope.

Improved plow steel rope (trade-marked by most manufacturers).See Grades, rope.

Incline ropes.-Ropes to operate cars on an incline haulage.

Independent wire rope core.-Wire rope used as the core of a larger rope.

Inner wires. - All wires of a strand except surface or cover wires.

Internally lubricated.-Wire rope or strand having all wires coated with lubricant.

Iron rope.-See Grades, rope.

IWRC.-Independent wire rope core.

Kink. - Sharp bend in a wire rope that permanently distorts the wires and strands.

Lagging. - External wood covering on a reel of rope or strand.

Lang lay rope.-Wire rope in which the wires in the strands and the strands in the rope are laid in the same direction.

Lay.-Manner in which wires are helically laid into strands or strands into rope.

Lead line.- That part of a rope tackle leading from the first or fast sheave to the drum.

Left lay:

Strand.-Strand in which the cover wires are laid in a helix having a left-hand pitch, similar to a left-hand screw. 


\section{Left lay-Continued}

Rope.-Rope in which the strands are laid in a helix having a left-hand pitch, similar to a left-hand screw.

Line.-Synonymous with Wire rope.

Locked-coil strand.-Smooth-surfaced strand composed of shaped wires laid in concentric layers around a center of round wires.

Loop.-See Eye or eye splice.

Marline-clad rope.-Rope with individual strands spirally wrapped with marline.

Marline spike.-Tapered steel pin used in splicing wire rope.

Messenger strand.-Galvanized strand or bronze strand used to support telephone and electrical cables.

Metallic cores.-See Core and IWRC.

Mild plow steel rope.-See Grades, rope.

Modulus of elasticity.-Mathematical quantity giving the ratio, within the elastic limit, between a definite range of unit stress on a wire rope, and the corresponding elongation.

Mooring lines.-Galvanized wire rope, usually $6 \times 12,6 \times 24$, or spring lay construction, for holding ships to dock.

Nonrotating wire rope. $-18 \times 7$ wire rope consisting of a $6 \times 7$ left lang lay inner rope covered by 12 seven-wire strands right regularlay.

Nonspinning wire rope.-See Nonrotating wire rope.

Open socket.-Wire rope fitting consisting of a "basket" and two "ears" with a pin.

Outer wires. - See Cover wires.

Peening.-Permanent distortion of outside wire in a rope caused by pounding.

Plow steel rope.-See Grades, rope.

Preece test.-A recognized standard of testing the galvanized coating on wire.

Preformed wire rope.-Wire rope in which the strands are permanently shaped, before fabrication into the rope, to the helical form they assume in the wire rope.

Preformed strand.- Strand in which the wires are permanently shaped, before fabrication into the strands, to the helical form they assume in the strand.

Prestressing.-Stressing a wire rope or strand before use under such a tension and for such a time that the constructional stretch is largely removed.

Proportional limit.-Considered synonymous with Elastic limit.

Reel.-The flanged spool on which wire rope or strand is wound for storage or shipment.

Regular lay rope.- Wire rope in which the wires in the strands and the strands in the rope are laid in opposite directions.

Reserve strength.- Strength represented by the inner wires of a wire rope.

Reverse bend.-Reeving of a wire rope over sheaves and drums so that it bends in opposite directions.

Reverse lay.-See Alternate lay.

Right lay:

Strand.-Strand in which the cover wires are laid in a helix having a right-hand pitch, similar to a right-hand screw. 
Rope.-Rope in which the strands are laid in a helix having a right-hand pitch, similar to a right-hand screw.

Rollers.-Relatively small-diameter cylinders or wide-faced sheaves for supporting ropes.

Rotary lines.-The wire rope on a rotary drilling rig which controls the position of the drill pipe and raises or lowers same.

Running rope.-Term used in describing $6 \times 12$ galvanized rope.

Safe working load.-Proper load which the rope may carry economically and safely.

Safety factor.-See Factor of safety.

Sag.- See Deflection.

Sand line.-See Bailing line.

Sash cord.-Term applied to small $6 \times 7$ wire ropes commonly made of iron, bronze, or copper wires.

Seale.-A strand construction having one size of cover wires with the same number of one size of wires in the inner layer, and each layer having the same length and direction of lay. Most common construction is one center wire, nine inner wires, and nine cover wires.

Seize.-To bind securely the end of a wire rope or strand with seizing wire or strand.

Seizing strand.-Small strand usually of seven wires made of soft annealed iron wire. See Seize.

Seizing wire.-A soft annealed iron wire. See Seize.

Serve.-To cover the surface of a wire rope or strand with a wrapping of fiber cord.

Sewing wires.-See Flat rope.

Shackle.-A U-shaped fitting with pin.

Sheave.-A grooved pulley for use with rope.

Siemens-Martin strand.--A grade of galvanized strand.

Single galvanized strand.- Strand made in the "common grade" of wiped galvanized wire. See Common strand.

Slings. - Wire ropes made into forms, with or without fittings, for handling loads and so made as to permit the attachment of an operating rope.

Slings, braided.-A very flexible sling composed of several individual wire ropes braided into a single sling.

Smooth coil strand. - Strand composed entirely of round wires, used as an aerial conveyor track cable.

Smooth-faced drum.-Drum with a plain face, not grooved.

Socket.-Type of wire rope fitting. See Bridge socket, Closed socket, Open socket, and Wedge socket.

Special flexible.-See Extra-flexible wire rope.

Spiral groove.-Groove which follows the path of a helix around the drum, as the thread of a screw.

Splicing.-Interweaving of two ends of ropes so as to make a continuous or endless length without appreciably increasing the diameter. Also making a loop or eye in the end of a rope by tucking in the ends of the strands.

Spring lay rope.-Preformed cable-laid rope consisting of six ropes around a fiber core, each individual rope consisting of three galvanized steel strands and three fiber cords laid alternately around a fiber core. 
Stainless steel rope.-See Corrosion-resistant steel.

Standing rope.-See Guy line.

Steel-clad rope.-Rope with individual strands helically wrapped with flat steel wire.

Stirrup.-The U-bolt or eyebolt attachment on a bridge socket.

Stone sawing strand.-Usually a three-wire strand used in quarrying stone or slate.

Strand.-An arrangement of wires helically laid about an axis, or another wire or fiber center to produce a symmetrical section.

Strand center.-See Centers.

Strand core.-See Core.

Suspension bridge cables.-See Bridge cable.

Swaged fittings.-Fittings in which wire rope is inserted and attached by cold-flowing method.

Tag line.-A small wire rope used to prevent rotation of a load.

Tapered drum.--See Conical drum.

Tapering and welding.-Reducing the diameter of the end of a wire rope and welding it to facilitate reeving.

Thimble.-Grooved metal fitting to protect the eye of a wire rope.

Tiller rope.-A very flexible operating rope, commonly made by cable laying six $6 \times 7$ ropes around a fiber core.

Tinned wire.-Wire coated with tin.

Track cable.-The suspended wire rope or strand along which the carriers move on an aerial conveyor.

Traction rope.-Wire rope that propels the carriages on an aerial conveyor.

Traction steel.-A grade of wire rope used in elevator service. See Grades, rope.

Tramway.-Aerial conveying system for transporting multiple loads. Turnbuckle.--Device attached to wire rope for making limited adjustments in length. It consists of a barrel and right-and left-hand threaded bolts.

Warrington.-A strand construction in which one layer of wires, usually the outer, is composed of alternating large and small wires.

Wedge socket.-Wire rope fitting in which the rope end is secured by a wedge.

Wire (round).--Single continuous length of metal cold-drawn from a rod.

Wire (shaped).-A single continuous length of metal either colddrawn or cold-rolled from a rod.

Wire rope.-A group of strands laid helically around an axis or a core.

\section{EFFECTIVE DATE}

32. Having been passed through the regular procedure of the Commodity Standards Division, and approved by the acceptors hereinafter listed, this commercial standard was issued by the United States Department of Commerce, effective from February 1, 1949.

EDWIN W. Ely, Chief, Commodity Standards Division. 


\section{HISTORY OF PROJECT}

33. On March 19, 1948, the Wire Rope Institute requested the cooperation of the National Bureau of Standards in the establishment of a commercial standard for wire rope for export trade.

34. Following receipt of this request by the Bureau, copies of a proposed commercial standard for wire rope for export trade were circulated on May 5, 1948, to selected representatives of manufacturers and exporters for advance comment. All comments were carefully considered, after which the standard was adjusted in accordance with composite recommendations of those concerned, and circulated on August 4, 1948, to the trade for written acceptance.

35. Upon receipt of official acceptances estimated to represent a satisfactory majority of the production, by volume, for export, and in the absence of active valid opposition, the standard was promulgated on January 1, 1949, as Commercial Standard CS154E-49.

\section{STANDING COMMITTEE}

36. The following individuals comprise the membership of the standing committee, which is to review, prior to circulation for acceptance, revisions proposed to keep the standard abreast or progress. Comment concerning the standard and suggestions for revision may be addressed to any member of the committee or to the Commodity Standards Division, National Bureau of Standards, which acts as secretary for the committee.

A. J. Morgan (chairman), John A. Roebling's Sons Co., Trenton, N. J.

C. D. Meals, Bethlehem Steel Co., Williamsport, Pa.

E. S. Wellhofer, American Chain \& Cable Co., Wilkes-Barre, Pa.

W. P. Laseter, 19 Rector Street, New York, N. Y. (Representing the Wire Rope Export Trade Association).

J. L. Manson, American Steel \& Wire Co., New Haven, Conn. 


\section{APPENDIX}

DECIMAL AND MILLIMETER EQUIVALENTS OF PARTS OF AN INCH

\begin{tabular}{|c|c|c|c|c|c|c|c|c|c|c|c|c|c|}
\hline 4ths & 8ths & 16ths & $32 \mathrm{ds}$ & 64ths & $\begin{array}{l}\text { Decimals } \\
\text { of an inch }\end{array}$ & $\begin{array}{l}\text { Milli- } \\
\text { meters }\end{array}$ & 4ths & 8ths & 16ths & $32 \mathrm{ds}$ & 64ths & $\begin{array}{l}\text { Decimals } \\
\text { of an inch }\end{array}$ & $\begin{array}{l}\text { Milli- } \\
\text { meters }\end{array}$ \\
\hline 1 & 2 & 2 & $\begin{array}{r}1 \\
2 \\
3 \\
4 \\
5 \\
6 \\
7 \\
8 \\
9 \\
10 \\
11 \\
12 \\
13 \\
14 \\
15 \\
16\end{array}$ & $\begin{array}{r}1 \\
2 \\
3 \\
4 \\
5 \\
6 \\
7 \\
8 \\
9 \\
10 \\
11 \\
12 \\
13 \\
14 \\
15 \\
16 \\
17 \\
18 \\
19 \\
20 \\
21 \\
22 \\
23 \\
24 \\
\\
25 \\
26 \\
27 \\
28 \\
29 \\
30 \\
31 \\
32\end{array}$ & $\begin{array}{l}0.015625 \\
.03125 \\
.046875 \\
.0625 \\
\\
.078125 \\
.09375 \\
.109375 \\
.1250 \\
.140625 \\
.15625 \\
.171875 \\
.1875 \\
.203125 \\
.21875 \\
.234375 \\
.2500 \\
.265625 \\
.28125 \\
.296875 \\
.3125 \\
.328125 \\
.34375 \\
.359375 \\
.375 \\
.390625 \\
.40625 \\
.421875 \\
.4375 \\
.453125 \\
.46875 \\
.484375 \\
.500\end{array}$ & \begin{tabular}{|c|}
0.397 \\
.794 \\
1.191 \\
1.588 \\
\\
1.984 \\
2.381 \\
2.778 \\
3.175 \\
\\
3.572 \\
3.969 \\
4.366 \\
4.763 \\
\\
5.159 \\
5.556 \\
5.953 \\
6.350 \\
6.747 \\
7.144 \\
7.541 \\
7.938 \\
8.334 \\
8.731 \\
9.128 \\
9.525 \\
9.922 \\
10.32 \\
10.72 \\
11.11 \\
11.51 \\
11.91 \\
12.30 \\
12.70
\end{tabular} & 4 & 7 & 14 & $\begin{array}{l}17 \\
18 \\
19 \\
20 \\
21 \\
22 \\
23 \\
24 \\
25 \\
26 \\
27 \\
28 \\
29 \\
30 \\
31 \\
32\end{array}$ & $\begin{array}{l}33 \\
34 \\
35 \\
36 \\
\\
37 \\
38 \\
39 \\
40 \\
41 \\
42 \\
43 \\
44 \\
45 \\
46 \\
47 \\
48 \\
49 \\
50 \\
51 \\
52 \\
53 \\
54 \\
55 \\
56 \\
57 \\
58 \\
59 \\
60 \\
61 \\
62 \\
63 \\
64\end{array}$ & $\begin{array}{l}.515625 \\
.53125 \\
.546875 \\
.5625 \\
.578125 \\
.59375 \\
.609375 \\
.625 \\
.640625 \\
.65625 \\
.671875 \\
.6875 \\
.703125 \\
.71875 \\
.734375 \\
.750 \\
.765625 \\
.78125 \\
.796875 \\
.8125 \\
.828125 \\
.84375 \\
.859375 \\
.875 \\
.890625 \\
.90625 \\
.921875 \\
.9375 \\
.953125 \\
.96875 \\
.984375 \\
1\end{array}$ & $\begin{array}{l}13.10 \\
13.49 \\
13.89 \\
14.29 \\
14.68 \\
15.08 \\
15.48 \\
15.88 \\
16.27 \\
16.67 \\
17.07 \\
17.46 \\
17.86 \\
18.26 \\
18.65 \\
19.05 \\
19.45 \\
19.84 \\
20.24 \\
20.64 \\
\\
21.03 \\
21.43 \\
21.83 \\
22.23 \\
\\
22.62 \\
23.02 \\
23.42 \\
23.81 \\
24.21 \\
24.61 \\
25.00 \\
25.40\end{array}$ \\
\hline
\end{tabular}




\section{ACCEPTANCE OF COMMERCIAL STANDARD}

If acceptance has not previously been filed, this sheet properly filled in, signed, and returned will provide for the recording of your organization as an acceptor of this commercial standard.

\section{Date}

Commodity Standards Division,

National Bureau of Standards,

Washington 25, D. C.

\section{Gentlemen:}

We believe that the Commercial Standard CS154E-49 constitutes a useful standard of practice, and we individually plan to utilize it as far as practicable in the

$$
\text { production }^{1} \quad \text { export }^{1} \quad \text { testing }^{1}
$$

of wire rope (export classification).

We reserve the right to depart from it as we deem advisable.

We understand, of course, that only those articles which actually comply with the standard in all respects can be identified or labeled as conforming thereto.

Signature of authorized officer

Name and title of above officer

Organization

Street address

City, zone, and State

1 Underscore which one. Please see that separate acceptances are flled for all subsidiary companies and affiliates which should be listed separately as acceptors. In the case of related interests, trade associations, trade papers, etc., desiring to record their general support, the words "General Support" should be added after the signature. 


\section{TO THE ACCEPTOR}

The following statements answer the usual questions arising in connection with the acceptance and its significance:

1. Enforcement.-Commercial standards for exports are commodity specifications voluntarily established by mutual consent of those concerned. They present a common basis of understanding between the producer, distributor, and consumer and should not be confused with any plan of governmental regulation or control. The United States Department of Commerce has no regulatory power in the enforcement of their provisions, but their provisions through usage soon become established as trade customs, and are made effective through incorporation into sales contracts by means of labels, invoices, and the like.

2. The acceptor's responsibility.-The purpose of commercial standards is to establish for specific commodities, nationally recognized grades or consumer criteria, and the benefits therefrom will be measurable in direct proportion to their general recognition and actual use. Instances will occur when it may be necessary to deviate from the standard and the signing of an acceptance does not preclude such departures; however, such signature indicates an intention to follow the commercial standard where practicable, in the production or exportation of the article in question.

3. The Department's responsibility.-The major function performed by the Department of Commerce in the voluntary establishment of commercial standards for exports on a Nation-wide basis is fourfold: first, to act as an unbiased coordinator to bring all interested parties together for the mutually satisfactory adjustment of trade standards; second, to supply such assistance and advice as past experience with similar programs may suggest; third, to canvass and record the extent of acceptance and adherence to the standard on the part of producers and exporters; and fourth, after acceptance, to publish and promulgate the standard for the information and guidance of buyers and sellers of the commodity.

4. Announcement and promulgation.-When the standard for exports has been endorsed by a satisfactory majority of production in the absence of active, valid opposition, the success of the project is announced. If, however, in the opinion of the standing committee or the Department of Commerce, the support of any standard is inadequate, the right is reserved to withhold promulgation and publication. 


\section{ACCEPTORS}

The organizations listed have individually accepted this standard for use as far as practicable in the production, export, or testing of wire rope (export classification). In accepting the standard they reserved the right to depart therefrom as they individually deem advisable. It is expected that articles which actually comply with the requirements of this standard in all respects will be regularly identified as conforming thereto, and that purchasers will require such evidence of conformity.

\section{ASSOCIATIONS}

(General Support)

Wire Rope Export Trade Association, New York, N. Y.

Wire Rope Institute, Washington, D. C.

\section{FIRMS}

Aircraft Mechanics, Inc., Colorado Springs, Colo. American Chain \& Cable Co., Inc., Wilkes-Barre, $\mathrm{Pa}$.

American Steel \& Wire Co., Cleveland, Ohio.

Bethlehem Steel Co., Bethlehem, Pa.

Broderick \& Bascom Rope Co., St. Louis, Mo

California Wire Cloth Corp., Oakland, Calif.

Edwards, E. H., Co., San Francisco, Calif.

Jones \& Laughlin Steel Corp., Pittsburgh, Pa.

Leschen, A., \& Sons Rope Co., St. Louis, Mo

LeTourneau, R. G., Inc., Peoria, Ill.

Macwhyte Co., Kenosha, Wis.

Pacific Wire Rope Co., Los Angeles, Calif.

Pagel, Horton \& Co., Inc., New York, N. Y.

Paulsen-Webber Cordage Corp., New York, N. Y.

\section{FIRMS-Continued}

Pennsylvania Wire Rope Corp., Williamsport, Pa. Revolvator Co., North Bergen, N. J.

Rochester Ropes, Inc Culpeper, Va.

Roebling's, John A., Sons Co., Trenton, N. J.

Sears, Roebuck \& Co., Chicago, Ill.

Shepard Elevator Co., The, Cincinnati, Ohio.

Sunbury Wire Rope Manufacturing Co., Sunbury, $\mathrm{Pa}$.

Union Oil Co. of California, Los Angeles, Calif. (General support.)

Union Wire Rope Corp., Kansas City, Mo.

United States Steel Export Co., New York, N. Y.

Upson-Walton Co., The, Cleveland, Ohio.

Wickwire Spencer Steel Division, The Colorado Fucl \& Iron Corp., Palmer, Mass.

Wire Rope Manufacturing \& Equipment Co. Seattle, Wash.

\section{UNITED STATES GOVERNMEN'T}

Agriculture, U. S. Department of, Division of Purchase, Sales \& Traffic, Washington, D. C

\section{COMMERCIAL STANDARDS}

CS No.

Item

$0-40$. Commercial standards and their value to business (third cdition).

1-42. Clinical thermometers (third edition).

2-30. Mopsticks.

3-40. Stoddard solvent (third cdition).

4-29. Staple porcelain (all-clay) plumbing fixtures.

5-46. Pipe nipples, brass, copper, steel, and wrought-iron (second edition).

6-31. Wrought-iron pipe nipples (second edition). Superseded by CS $5-46$.

7-29. Standard weight malleable iron or steel screwed unions.

8-41. Gage blanks (third edition).

9-33. Builders' template hardware (second edition).

10-29. Brass pipe nipples. Superseded by CS5-46.

11-41. Moisture regains of cotton yarns (second edition).

12-48. Fuel oils (sixth edition).

13-44. Dress patterns (fourth edition).

14-43. Boys' button-on waists, shirts, junior and sport shirts (made from woven fabries) (third edition).

15-46. Men's pajama sizes (made from woven fabrics) (third edition).

16-29. Wall paper.

17-47. Diamond core drill fittings (fourth edition).

18-29. Hickory golf shafts.

19-32. Foundry patterns of wood (second edition)

20-47. Staple vitreous china plumbing fixtures (fourth edition).

21-39. Interchangeable ground-glass joints, stopcocks, and stoppers (fourth edition).

22-40. Builders' hardware (nontemplate) (second edition).

23-30. Feldspar.

24-43. Screw threads and tap-drill sizes.

25-30. Special serew threads. Superseded by CS24-43.
CS No. Item

26-30. Aromatic red cedar closet lining.

27-36. Mirrors (second edition).

28-46. Cotton fabric tents, tarpaulins, and covers (second edition).

29-31. Staple seats for water-closet bowls

30-31. Colors for sanitary ware. (Withdrawn as commercial standard March 15, 1948.)

31-38. Wood shingles (fourth edition).

$32-31$. Cotton cloth for rubber and pyroxylin coating.

33-43. Knit underwear (exclusive of rayon) (second edition).

34-31. Bag, case, and strap leather.

35-47. Hardwood plywood (third edition).

36-33. Fourdrinier wire cloth (second edition).

37-31. Steel bonc plates and screws.

38-32. Hospital rubber sheeting.

39-37. Wool and part wool blankets (second edition). (Withdrawn as commercial standard, July 14,1941 .)

40-32. Surgeons' rubber gloves.

41-32. Surgeons' latex gloves.

42-43. Structural fiber insulating board (third edition).

43-32. Grading of sulphonated oils

44-32. Apple wraps.

45-48. Douglas fir plywood (eighth edition).

46-40. Hosiery lengths and sizes (third edition).

47-34. Marking of gold-filled and rolled-gold-plate articles other than watchcases.

48-40. Domestic burners for Pennsylvania anthracite (underfeed type) (second edition).

49-34. Chip board, laminated chip board, and miscellaneous boards for bookbinding purposes.

50-34. Binders board for bookbinding and other purposes.

51-35. Marking articles made of silver in combination with gold. 
CS No.

Item

52-35. Mohair pile fabrics (100-percent mohair plain velvet, 100-percent mohair plain frieze, and 50-percent mohair plain frieze)

53-35. Colors and finishes for cast stone.

54-35. Mattresses for hospitals.

55-35. Mattresses for institutions.

56-49. Oak flooring (third edition).

$56 \mathrm{E}-41$. Oak flooring (exports).

57-40. Book cloths, buckrams, and impregnated fabrics for bookbinding purposes except library bindings (second edition).

$58-36$. Woven elastic fabries for use in overalls (overall elastic webbing)

59-44. Textiles-testing and reporting (fourth edition).

60-48. Hardwood dimension lumber (second edition).

61-37. Wood-slat venetian blinds.

62-38. Colors for kitchen accessories.

63-38. Colors for bathroom accessories.

64-37. Walnut veneers.

65-43. Methods of analysis and of reporting fiber composition of textile products (second edition).

66-38. Marking of articles made wholly or in part of platinum.

67-38. Marking articles made of karat gold.

68-38. Liquid hypochlorite disinfectant, deodorant, and germicide.

69-38. Pine oil disinfectant.

70-41. Phenolic disinfectant (emulsifying typc) (second edition) (published with CS71-41).

71-41. Phenolic disinfectant (soluble type) (second edition) (published with CS70-41)

72-38. Household insecticide (liquid spray type).

73-48. Old growth Douglas fir, Sitka spruce, and Western hemlock standard stock doors (fourth edition).

74-39. Solid hardwood wall paneling.

75-42. Automatic mechanical draft oil burners designed for domestic installations (second edition).

76-39. Hardwood interior trim and molding.

77-48. Enameled cast-iron plumbing fixtures (second edition).

78-40. Ground-and-polished lenses for sun glasse (second edition) (published with CS79-40).

79-40. Blown, drawn, and dropped lenses for sun glasses (second edition) (published with CS78-40).

80-41. Electric direction signal systems other than semaphore type for commercial and other vehicles subject to special motor vehicle laws (after market).

81-41. Adverse-weather lamps for vehicles (after market).

82-41. Inner-controlled spotlamps for vehicles (after market).

83-41. Clearance, marker, and identification lamps for vehicles (after market).

84-41. Electric tail lamps for vehicles (after market).

85-41. Electric license-plate lamps for vehicles (after market).

86-41. Electric stop lamps for vehicles (after market).

87-41. Red electric warning lanterns.

88-41. Liquid burning flares.

89-40. Hardwood stair treads and risers.

90- (Reserved for power shovels and cranes).

90E-47. Power cranes, and shovels, convertible fullrevolving type; crawler, truck, and wheel mounted; including clamshell, dragline, lifting crane, hoe, pile driver, and skimmer scoop operating equipment (export classifications) (second edition)

91-41. Factory-fitted Douglas fir entrance doors.

92-41. Cedar, cypress and redwood tank stock lumber.

93-41. Portable electric drills (exclusive of high fre quency).

94-41. Calking lead.

95-41. Lead pipe.

96-41. Lead traps and bends.
CS No.

Item

97-42. Electric supplementary driving and passing lamps for vehicles (after market).

98-42. Artists' oil paints.

99-42. Gas floor furnaces-gravity circulating type.
100-47. Porcelain-enameled steel utensils (third edition).

101-43. Flue-connected oil-burning space heaters equipped with vaporizing pot-type burners.

102-. (Reserved for Diesel and fuel-oil engines).

102E-42. Diesel and fuel oil engines (export classifications).

103-48. Rayon jacquard velour (with or without other decorative yarn) (second edition).

104-46. Warm-air furnaces equipped with vaporizing pot-type oil burners (second edition).

105-48. Mineral wool insulation for low temperatures (second edition).

106-44. Boys' pajama sizes (woven fabrics) (second edition).

107-45. Commercial electric-refrigeration condensing units (second edition). (Withdrawn as commercial standard September 4, 1947.)

108-43. Treading automobile and truck tires.

109-44. Solid-fuel-burning forced-air furnaces.

110-43. Tire repairs-vulcanized (passenger, truck. and bus tires).

111-43. Earthenware (vitreous-glazed) plumbing fixtures.

112-43. Homogeneous fiber wallboard.

113-44. Oil-burning floor furnaces equipped with vaporizing pot-type burners.

114-43. Hospital sheeting for mattress protection.

115-44. Porcelain-enameled tanks for domestic use.

116-44. Bituminized-fibre drain and sewer pipe.

117-44. Mineral wool; blankets, blocks, insulating cement, and pipe insulation for heated in dustrial equipment.

118-44. Marking of jewelry and novelties of silver.

(E) 119-45.1 Dial indicators (for linear measurements).

120-48. Standard stock ponderosa pine doors (third edition).

121-45. Women's slip sizes (woven fabrics).

122-45. Western hemlock plywood.

123-45. Grading of diamond powder.

(E) 124-45.1 Master disks.

125-47. Prefabricated homes (second edition)

126-45. Tank mounted air compressors.

127-45. Self - contained mechanically refrigerated drinking water coolers.

128-45. Men's sport shirt sizes-woven fabrics (other than those marked with regular neckband sizes).

129-47. Materials for safety wearing apparel (second edition)

130-46. Color materials for art education in schools.

131-46. Industrial mineral wool products, all typestesting and reporting.

132-46. Hardware cloth.

133-46. Woven wire netting

134-46. Cast aluminum cooking utensils (metal composition).

135-46. Men's shirt sizes (exclusive of work shirts).

136-46. Blankets for hospitals (wool, and wool and cotton).

137-46. Size measurements for men's and boys' shorts (woven fabrics).

138-47. Insect wire screening.

139-47. Work gloves.

140-47. Testing and rating convectors

141-47. Sine bars, blocks, plates, and fixtures.

142-47. Automotive lifts.

143-47. Standard strength and extra strength perforated clay pipe.

144-47. Formed metal porcelain enameled sanitary ware.

145-47. Testing and rating hand-fired hot-watersupply boilers.

146-47. Gowns for hospital patients.

147-47. Colors for molded urea plastics.

148-48. Men's circular flat and rib knit rayon underwear.

149-48. Utility type house dress sizes.

1 Where “(E)" precedes the CS number, it indicates an emergency commercial standard, drafted under war conditions with a view toward early revision. 
CS No.

Item

150-48. Hot rolled rail steel bars (produced from Teesection rails).

151-48. Body measurements for the sizing of apparel for infants, babies, toddlers, and children (for the knit underwear industry).

152-48. Copper naphthenate wood-preservative.

153-48. Body measurements for the sizing of apparel
CS No.

Item

154-. (Reserved for wire rope.)

$154 \mathrm{E}-49$. Wire rope (export classification).

155-49. Body measurements for the sizing of apparel for boys (for the knit-underwear industry).

156-49. Colors for polystyrene plastics.

157-49. Ponderosa pine and sugar pine plywood.

158-49. Model forms for girls' apparel.

Noтice.-Those interested in commercial standards with a view toward accepting them as a basis of everyday practice may secure copies of the above standards, while the supply lasts, by addressing the Commodity Standards Division, National Bureau of Standards, Washington 25, D. C. 
